\title{
Regulation of the Unfolded Protein Response through ATF4 and FAM129A in Prostate Cancer
}

Running title: Stress response as a target in prostate cancer

Nora Pällmann ${ }^{a}$, Marte Livgård ${ }^{a, b}$, Martina Tesikova $^{a}$, Hatice Zeynep Nenseth ${ }^{a}$, Erman Akkus $^{a}$, Jørgen Sikkeland ${ }^{a, b}$, Yixin Jin ${ }^{a, 1}$, Dogukan Koc ${ }^{a, 2}$, Omer Faruk Kuzu ${ }^{a}$, Manohar Pradhan ${ }^{b}$,

Håvard E. Danielsen ${ }^{b, c, d}$, Nermin Kahraman ${ }^{\mathrm{e}}$, Hamada M. Mokhlis ${ }^{\mathrm{e}}$, Bulent Ozpolat ${ }^{\mathrm{e}}$, Partha P.

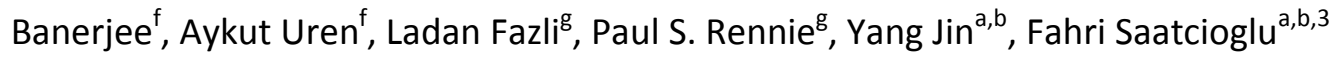

${ }^{a}$ Department of Biosciences, University of Oslo, Oslo, Norway

${ }^{b}$ Institute for Cancer Genetics and Informatics, Oslo University Hospital, Oslo, Norway

${ }^{\mathrm{c}}$ Department of Informatics, University of Oslo

${ }^{\mathrm{d}}$ Nuffield Division of Clinical Laboratory Sciences, University of Oxford, United Kingdom

${ }^{\mathrm{e}}$ Department of Experimental Therapeutics, MD Anderson Cancer Center, Houston, TX, USA

${ }^{f}$ Lombardi Comprehensive Cancer Center, Georgetown University, Washington, DC, USA

${ }^{g}$ The Vancouver Prostate Centre, Vancouver, BC, Canada

${ }^{1}$ Present address: Department of Cancer Immunology, Institute for Cancer Research, Oslo University Hospital, Oslo, Norway

${ }^{2}$ Present address: Department of Child and Adolescent Psychiatry, Faculty of Medicine, Dokuz Eylul University, Izmir, Turkey 
${ }^{3}$ Corresponding author. University of Oslo, Postboks 1066, Blindern, 0316 Oslo, Norway, Phone: +47 22854569; Fax: +47 22857207; E-mail: fahris@ibv.uio.no

The authors declare no potential conflicts of interest. This work was supported by Norwegian Research Council grant 193337, Norwegian Cancer Society grant 419204, and Health South East Norway grant 36024.

\section{Abstract}

Cancer cells exploit many of the cellular adaptive responses to support their survival needs. One such critical pathway in eukaryotic cells is the unfolded protein response (UPR) that is important in normal physiology as well as disease states, including cancer. Since UPR can serve as a lever between survival and death, regulated control of its activity is critical for tumor formation and growth although the underlying mechanisms are poorly understood. Here we show that one of the main transcriptional effectors of UPR, activating transcription factor 4 (ATF4), is essential for prostate cancer ( $\mathrm{PCa}$ ) growth and survival. Using systemic unbiased gene expression and proteomic analyses, we identified a novel direct ATF4 target gene, family with sequence similarity 129 member A (FAM129A), which is critical in mediating ATF4 effects on prostate tumorigenesis. Interestingly, FAM129A regulated both PERK and elF2 $\alpha$ in a feedback loop that differentially channeled the UPR output. ATF4 and FAM129A protein expression is increased in patient PCa samples compared with benign prostate. Importantly, in vivo therapeutic silencing of ATF4-FAM129A axis profoundly inhibited tumor growth in a preclinical PCa model. These data support that one of the canonical UPR branches, through ATF4 and its target gene FAM129A, is required for PCa growth and thus may serve as a novel therapeutic target. 


\section{Keywords}

ATF4 / FAM129A / unfolded protein response / prostate cancer/ feedback regulation

\section{Introduction}

Cells have developed numerous strategies to cope with stress, resulting in either survival or cell death. Cellular stress pathways are now recognized as important players in cancer development [1, 2]. Cancer cells can survive under highly stressful conditions, such as a hypoxic or nutrient-deprived tumor microenvironment, which involves activation of stress-related signaling pathways leading to survival, while avoiding those that induce cell death.

One of the most critical pathways in this regard is the unfolded protein response (UPR) which is activated by a variety of stressful conditions. One of the canonical UPR pathways is mediated by the phosphorylation of eukaryotic translation initiation factor $2 \alpha$ (elF $2 \alpha$ ), which in turn regulates protein translation. In mammalian cells, upon endoplasmic reticulum (ER) stress, elF $2 \alpha$ phosphorylation is catalyzed by ER protein kinase RNA-activated (PKR)-like ER kinase (PERK). elF2 $\alpha$ phosphorylation causes an inhibition of global translation, but also translational activation of a specific set of mRNAs containing short upstream open reading frames (UORFs) [3]. One of these mRNAs encodes activating transcription factor 4 (ATF4).

ATF4 belongs to the basic-region leucine zipper (bZIP) family of transcription factors and functions as heterodimers with other bZIP proteins, such as c-Fos, c-Jun, or CCAAT/enhancer-binding proteins (C/EBPs) binding to C/EBP-ATF response elements (CARE) in the promoter regions of target genes and activate transcription [4]. ATF4 acts as a master regulator controlling the transcription of key genes essential for adaptive functions and modulates cellular fate in response to stress [5]. Conversely, ATF4 induces apoptosis under chronic stress of sufficient magnitude [6]. The mechanisms that modulate this dual role of ATF4 are incompletely understood and may include different ATF4 heterodimerization partners, the cellular context, as well as posttranslational modifications of ATF4 
and epigenetic modifications [5].

ATF4 expression is deregulated in various cancers and correlates with a more aggressive phenotype or unfavorable prognosis (for reviews, see $[4,5]$ ). Consistently, ATF4 is implicated in diverse steps of tumorigenesis. However, little is known about the potential role of ATF4 in prostate cancer (PCa), which is the most frequently diagnosed non-cutaneous cancer in men and the second most common cause of cancer death [7]. The prostate is a major secretory organ and is particularly dependent on optimal ER functioning. Consistently, ER stress and the UPR are also critical in PCa [8, 9].

Here, we show that ATF4 has essential pro-survival functions in PCa cells in vitro and in vivo through direct activation of a broad range of genes. Among these, we identified family with sequence similarity 129 member A (FAM129A) as a novel ATF4-C/EBP $\beta$ target gene that modulates the PERKelF2 $\alpha$ pathway at two different steps. Consistently, ATF4 and FAM129A expression is increased in human PCa and their nanoliposomal siRNA-mediated inhibition in a preclinical PCa model profoundly inhibited tumor growth, suggesting that both may serve as potential therapeutic targets. Additionally, FAM129A impacts elF2 $\alpha$ signaling by direct interactions with PERK.

\section{Results}

\section{ATF4 has a critical role in PCa growth both in vitro and in vivo}

We have recently found that ATF4 protein expression was increased upon androgen induction which is a central growth and survival pathway in PCa [9]. To directly assess the potential role of ATF4 in PCa cells, we used siRNA-mediated knockdown and evaluated possible effects on cell growth. ATF4specific siRNAs efficiently decreased ER stress-induced ATF4 expression by thapsigargin (Tg) in the PCa cell lines LNCaP and VCaP at both mRNA and protein levels (Fig. 1A and S1A). ATF4 knockdown significantly reduced cell growth rates as well as colony formation capacity compared with control cells (Fig. 1B-C and S1B-C). Similar results were obtained with $22 \mathrm{Rv} 1$ cells, a model of castration resistant PCa (CRPC) (Fig. S1D-F). Consistently, doxycycline-inducible shRNA-mediated ATF4 silencing 
in LNCaP cells resulted in reduced growth rates (Fig. S1G-J). In keeping with these data, ATF4 knockdown led to a significant decrease in the expression of proliferation markers (Ki67, PCNA) and several cyclins (A2, B1, D3 and E2) as well as a strong decrease in retinoblastoma protein (Rb) phosphorylation (Fig. 1D and S1K), indicating that ATF4 silencing leads to partial cell cycle arrest. In addition, ATF4 knockdown increased the expression of apoptosis markers cleaved poly-ADP ribose polymerase (PARP) and cleaved Caspase 3 in LNCaP cells upon treatment with tumor necrosis factor (TNF) related apoptosis-inducing ligand (TRAIL) and the PI3K inhibitor LY294002 (LY). This indicates that ATF4 loss sensitizes PCa cells to apoptosis (Fig. 1E) which is consistent with findings in other tumor cells [10].

The data presented above suggested that ATF4 promotes cell growth and survival in PCa. However, its role in tumorigenesis and its potential as a therapeutic target is not known. To assess this possibility, we used nanoliposomal siRNA delivery in a preclinical PCa model [11]. VCaP cells were subcutaneously injected into male nude mice. Once tumors were palpable, mice were given empty nanoliposomes or nanoliposome-encapsulated ATF4-specific siRNA by intraperitoneal injection and tumor growth was monitored over time. Consistent with the in vitro data presented above, treatment with ATF4-specific nanoliposomal siRNA remarkably inhibited tumor growth (Fig. 1F). These data suggest that ATF4 is a potential therapeutic target in PCa.

\section{ATF4 expression is up-regulated in human PCa specimens}

To evaluate ATF4 expression in human PCa and its potential association with clinical/pathological features, we analyzed a tissue microarray (TMA) containing benign prostate and PCa specimens for ATF4 expression by immunohistochemistry, as well as samples obtained at various stages after neoadjuvant hormone therapy (NHT). ATF4 expression was significantly increased in PCa compared to benign prostate and it was reduced following NHT (Fig. 1G-H). This is consistent with the previously described androgen regulation of ATF4 expression [9] and the importance of androgen signaling in PCa [12]. These data suggest that ATF4 is involved in PCa progression. 


\section{Global analysis of ATF4-mediated signaling pathways in PCa cells}

To identify ATF4 target genes that mediate the UPR outcome and to get insight into its mechanism of action in $\mathrm{PCa}$, we next performed global transcriptomic and proteomic expression profiling in parallel. ATF4 expression was induced by Tg in LNCaP cells that were treated with either ATF4specific siRNA or scrambled siRNA as control. Global transcriptomic and proteomic expression analyses were conducted to map gene expression changes upon loss of ATF4 expression (Fig. 2A, S2A and Table S1).

Analysis of the overlap by the two approaches revealed putative early and late ATF4 target genes as well as non-transcriptional ATF4 effects on gene expression (Fig. 2B). Many genes encoding amino acid synthesis enzymes, amino acid transporters, tRNA ligases, as well as tRNA exporters were significantly reduced upon ATF4 knockdown (Fig. S2B-G), consistent with previously identified ATF4 effects $[6,13]$. Regulation of all these pathways by ATF4 was validated for multiple genes by qPCR using two independent ATF4 siRNAs in LNCaP (Fig. S2B-E and S2H-I), VCaP (Fig. S2F) and 22Rv1 cells (Fig. S2G). These data show that ATF4 regulates important metabolic pathways in PCa cells.

To ensure that the observed effects on gene expression are indeed due to ATF4 knockdown, we performed rescue experiments using a lentiviral ATF4 expression vector. As shown in Fig. 2C, ATF4 reexpression specifically rescued expression of all tested genes upon ATF4 knockdown (one gene of each category is shown as an example), confirming that their expression is regulated by ATF4.

To determine whether ATF4 directly regulates these genes, we screened the sequences in the vicinity of the transcription start sites (TSSs) and identified a putative CARE sequence in the promoter, 5'untranslated or intronic regions in all cases (Table S2). Upon ATF4 activation, there was a significant increase in ATF4 binding to these elements in chromatin immunoprecipitation (ChIP) analyses (shown for GARS and SLC1A5, Fig. S2J-K). Conversely, there was a significant reduction in Tg-induced ATF4 occupancy at these sites upon siRNA-mediated ATF4 depletion (Fig. S2J-K). These data show that ATF4 directly regulates expression of these genes. 
ATF4 regulates transcription of target genes as a heterodimer with other bZIP members [14]. Interestingly, we observed that the expression of CCAAT/enhancer binding protein beta (CEBPB), one of the interaction partners of ATF4, was significantly downregulated upon ATF4 knockdown (Fig. 2D and Table S1) and rescued by ectopic ATF4 expression (Fig. S2L). Further analysis identified a putative CARE sequence in the $5^{\prime}$-UTR of the CEBPB gene (Fig. S2M). We showed that ATF4 directly binds to this CARE sequence (Fig. 2E). This is consistent with previous reports that indicated ATF4 binding to the CEBPB promoter in adipocytes [15] and exclusive co-localization of ATF4 with C/EBP $\beta$ during adipogenesis [16].

Taken together, these data firmly establish that ATF4 directly regulates specific gene expression programs in PCa cells which include at least one of its dimerization partners, C/EBP 3 .

\section{FAM129A is a novel ATF4 target gene in PCa}

To identify novel ATF4 target genes that may play an important role in PCa, we screened differentially expressed genes from the above analysis that were not previously connected to ATF4 and validated them. One of these was Family With Sequence Similarity 129 Member A (FAM129A) which encodes a protein without any significant homology to known functional domains [17]. Interestingly, recent global gene expression studies and ChIP-seq analysis suggested that FAM129A expression may be regulated by androgen signaling in PCa $[18,19]$. In addition, FAM129A was one of four genes that were differentially expressed in a whole blood mRNA screen in metastatic PCa samples [20]. We thus analyzed FAM129A expression further in PCa cells.

Given that ER stress induces ATF4 expression, we determined if FAM129A expression is also regulated upon ER stress in PCa cells. All ER stress inducers tested - Tg, Tunicamycin (Tm) and Brefeldin A (Bfa) - significantly upregulated FAM129A expression in LNCaP cells (Fig. 3A and S3A). Similar results were obtained in VCaP (Fig. S3B-C) and 22Rv1 cells (Fig. S3D-E). Consistent with being an ATF4 target, the time-dependent increase in FAM129A expression was delayed compared to ATF4 expression (Fig. S3A). In addition, siRNA-mediated ATF4 knockdown inhibited FAM129A expression in all three cell lines tested (Fig. 3B and S3F-G). Furthermore, ectopic expression of ATF4 rescued 
FAM129A expression (Fig. 3C-D). Consistently, bioinformatics analysis identified a putative ATF4 response element in intron 1 of the FAM129A gene (Fig. 3F). In ChIP experiments, ATF4 bound to this region upon ER stress that was abolished upon ATF4 silencing (Fig. 3E).

As shown above, ATF4 regulated expression of $C E B P B$, a possible dimerization partner of ATF4, in PCa cells (Fig. 2D-E and S2L-M). As ATF4-C/EBP $\beta$ dimers were suggested to signal adaptation responses in other settings [21], we tested whether C/EBP $\beta$ is required for transcriptional activation of FAM129A by ATF4. CEBPB knockdown significantly reduced FAM129A expression; concomitant ATF4 and CEBPB silencing had an additive effect in reducing FAM129A expression (Fig. S4A). Furthermore, consistent with its role in regulating FAM129A expression, C/EBP $\beta$ knockdown significantly reduced ATF4 binding to the CARE sequence in the FAM129A gene, suggesting that it is the ATF4-C/EBP $\beta$ dimer that binds to this site and regulates FAM129A expression (Fig. S4B), as opposed to other ATF4 targets that were not affected (Fig. S4C-E). Taken together, these data establish that FAM129A is a novel direct ATF4-C/EBP $\beta$ target gene in PCa cells whose expression is activated upon ER stress.

\section{FAM129A stimulates PCa cell growth by inhibiting senescence}

The data presented above suggested that FAM129A may mediate some of the ATF4 effects and itself may be important for PCa growth. In support of this, siRNA-mediated FAM129A knockdown (Fig. 4A) significantly decreased cell growth and colony formation of LNCaP, VCaP and 22Rv1 cells in vitro (Fig. 4B-C and S5A-B). Consistently, FAM129A knockdown led to a significant decrease in the expression of the proliferation markers Ki67 and PCNA (Fig. 4D), suggesting that FAM129A may represent an important mediator of ATF4 function in PCa cells.

Since it was previously reported that FAM129A inhibits apoptosis in mouse embryonic fibroblasts (MEFs) [22], we analyzed if FAM129A silencing induced apoptosis in PCa cells. There was no significant change in the expression of the apoptosis markers cleaved PARP and cleaved Caspase 3 (Fig. 4E), indicating that FAM129A does not affect apoptosis in PCa cells. 
FAM129A knockdown also led to a significant decrease in the expression of the $G 2$ and $M$ phasedriving cyclins A2, B1 and B2 (Fig. 4F) and decreased expression of the transcription factors E2F1 and E2F2 (Fig. 4F) and phosphorylation of Rb (Fig. 4G), indicating that FAM129A silencing leads to partial cell cycle arrest, similar to our observation for ATF4 (Fig. 1D). Expression of the cell cycle inhibitors p21/Cip1 and p15/INK4B was increased upon FAM129A knockdown (Fig. 4H). Furthermore, we observed morphological changes upon FAM129A silencing: cells became large, flat and vacuolized, characteristics of senescence (Fig. 4I). An increase in the number of senescence-associated ßgalactosidase positive cells (Fig. 4J and S5C) and a decrease in Lamin B1 (LMNB1) expression (Fig. S5D), confirmed induction of senescence upon FAM129A silencing.

Taken together, FAM129A functions as a pro-survival factor in PCa cells by inhibiting senescence, promoting cell cycle progression, and leading to cell survival under stress.

\section{Therapeutic targeting of FAM129A inhibits PCa growth in vivo}

To assess the therapeutic potential of FAM129A inhibition, we performed xenograft experiments in immunodeficient mice. VCaP cells were subcutaneously injected into nude mice and when tumors were palpable, mice were intraperitoneally injected with nanoliposomes containing either FAM129A siRNA or empty nanoliposomes and tumor growth was monitored. As shown in Fig. 5A, whereas tumors continued to grow rapidly in mice injected with the empty nanoliposomes, there was a dramatic inhibition of tumor growth upon injection of nanoliposomes containing FAM129A-specific siRNA. These data suggest that FAM129A is a novel therapeutic target for PCa treatment.

\section{FAM129A is up-regulated in human PCa}

It was recently suggested that androgen signaling plays a role in FAM129A expression $[18,19]$. We thus assessed whether FAM129A expression was induced by androgen treatment. Indeed, there was a strong increase in FAM129A expression upon treatment of LNCaP cells with the synthetic androgen R1881 (Fig. 5B), suggesting that FAM129A plays a role in PCa and possibly in the normal prostate. Consistently, FAM129A mRNA was expressed at medium-high levels in the prostate compared to various normal human tissues (Fig. S6A). 
Previous work has shown that FAM129A expression is upregulated in a number of cancer types [2325]. In light of these findings and the data presented above, we examined human PCa specimens for FAM129A expression using immunohistochemistry. FAM129A expression was significantly increased in PCa compared with benign prostate specimens (Fig. 5C-D). Interestingly, FAM129A expression was also higher in hyperplastic areas of the tumor specimens compared with areas with slower growth (Fig. 5E). Consistently, Kaplan-Meier estimates of recurrence free survival in 463 prostate cancer patients showed that the presence of FAM129A IHC staining in luminal tumor cells was linked to poor survival (Fig. 5F). In addition, we found that ATF4 and FAM129A are colocalized in consecutive sections of prostate cancer specimens (Fig. S6B). These data, together with those presented above, suggest that FAM129A is a potential therapeutic target in PCa.

In order to further evaluate the effects of ATF4 and FAM129A on prostate cancer growth, three different LuCaP patient-derived xenograft (PDX) models (LuCaP 23.1, LuCaP 86.2 and LuCaP 136) were used to develop organoids in the presence of control, ATF4-specific or FAM129A-specific siRNA and knockdown was confirmed by Western analysis (Fig. S6C). Treatment with ATF4 or FAM129A siRNAs induced extensive cell death (Fig. 6A and 6B) in all three LuCaP organoids compared to control siRNA treated ones. These results are consistent with the data presented above and suggest that ATF4 and FAM129A are potential drivers of PCa.

\section{FAM129A differentially affects PERK and elF2 $\alpha$ phosphorylation}

Given the significant impact of FAM129A on PCa growth in vitro and in vivo, we explored the potential molecular mechanisms of its action. To investigate FAM129A intracellular localization we co-expressed FAM129A together with markers for ER, lysosomes, or mitochondria (Fig. S6D). FAM129A displayed strong colocalization with ER, but not with lysosomes and mitochondria. Interestingly, FAM129A also displayed some plasma membrane localization suggesting that it may function in different sites of the cell.

Previous work has shown that FAM129A positively affects the protein translation machinery in MEFs by regulating phosphorylation of key proteins, including elF2 $\alpha$ [17]. Consistently, FAM129A 
knockdown increased elF $2 \alpha$ phosphorylation and decreased that of eukaryotic translation initiation factor 4E-binding protein 1 (4E-BP1) in LNCaP cells (Fig. 7A). This suggests that FAM129A promotes the anabolic activity needed in a PCa tumor that is under ER stress by limiting the effects of elF2 $\alpha$ phosphorylation.

Interestingly, there was decreased protein expression of ATF4 and the ATF4 target gene CHOP upon FAM129A knockdown despite increased elF2 $\alpha$ phosphorylation (Fig. 7A). ATF4 and CHOP mRNA levels were not affected by FAM129A knockdown (Fig. S7A), suggesting that posttranscriptional effects occurring after translation initiation by elF $2 \alpha$ may be involved. This was supported by experiments using the proteasome inhibitor MG132 where ATF4 protein expression was reduced in the absence of FAM129A (Fig. S7B), suggesting that FAM129A has a role in its translation rather than proteasomal degradation.

We next considered potential molecular mechanism(s) for the feedback regulation of the elf2 $\alpha$ ATF4-FAM129A axis. Since elF2 $\alpha$ is known to be regulated by distinct protein kinases in response to various stress stimuli [26], we determined if one of the upstream elF2 $\alpha$ kinases is affected by FAM129A. We thus performed double knockdown experiments where we silenced FAM129A as well as one of the three elF2 $\alpha$ kinases - PERK, GCN2, and PKR - that are known to be expressed in PCa cells. As shown in Fig. 7B, only FAM129A+PERK knockdown led to a significant reduction in the FAM129A knockdown-mediated upregulation of elF2 $\alpha$ phosphorylation. This suggests that FAM129A effects are largely mediated by PERK. Interestingly, ectopic expression of FAM129A significantly increased p-PERK levels in a dose dependent fashion (Fig. 7C and S7C). Similar results were obtained in the CRPC cell lines 22Rv1 and C4-2B (Fig. S7D). Consistently, Tg-induced p-PERK levels significantly decreased upon FAM129A knockdown (Fig. 7D). Under conditions where p-PERK levels were increased, p-elF2 $\alpha$ levels were not significantly affected (Fig. 7C and Fig. S7E-F). These data suggest that FAM129A increases PERK phosphorylation but inhibits that of elF2awhile other UPR branches are not significantly affected by FAM129A knockdown (Fig. S7GF). 
We next considered whether there may be direct interactions between PERK and FAM129A. Predictive bioinformatics analysis supported this possibility (data not shown). Consistently, immunoprecipitation (IP)-Western experiments revealed direct PERK-FAM129A interactions in HEK293T cells, which was increased by Tg stimulation (Fig. 7E). This interaction was mediated by the N-terminal region of FAM129A (aa 1-277) (Fig. 7F and 7H), which is the most conserved region between species and therefore thought to encompass functionally important moieties [17]. Conversely, ER luminal domain of PERK (Fig. 7G) was not required for FAM129A interaction (Fig. 7H), suggesting that the FAM129A-PERK interactions occur in the cytosolic side of the ER membrane. Consistently, confocal microscopy showed that PERK and FAM129A are largely colocalized inside the cell (Fig. 7I-J). These data suggest that FAM129A physically interacts with PERK and regulates its activity.

\section{Discussion}

Stress response pathways are often activated in disease states and have critical roles in cancer biology $[1,2]$. However, the stress response is a double-edged sword leading to either survival or cell death; how this decision is made is largely unknown. Identification of factors that modulate the stress response are thus vital to design new therapeutic approaches against cancer. Here we identify the ATF4-FAM129A signaling, with feedback regulation to PERK, as an important player in this regard.

The potential role of ATF4 beyond metabolic regulation in PCa has not been studied to date. The data we present here establish ATF4 as a critical factor for PCa. In contrast to that observed in MEFs [27], amino acid supplementation and antioxidants did not rescue the effect of ATF4 depletion in PCa cells (data not shown). Thus, we hypothesized that other ATF4 target genes may contribute to its function as a survival factor in PCa and performed global transcriptomics and proteomics analysis, confirming select targets by ChIP analysis; in this process, we identified FAM129A as a direct ATF4 target gene. 
ATF4, together with C/EBP $\beta$, binds to a CARE sequence in the promoter of the FAM129A and directly regulates FAM129A transcription (Fig. 3). Combined with the observation that CEBPB gene transcription itself is activated by ATF4, this constitutes a feed-forward regulation of FAM129A. We suggest that this allows for temporal activation of target gene expression that can be controlled rapidly and precisely, such as during ER stress, so that the pro-survival, but not the pro-apoptotic aspects, are activated.

FAM129A knockout mice do not display an obvious phenotype [17] suggesting that it is not required for normal mouse growth and development, whereas it has a critical role for PCa cell growth (Fig. 1). As in HeLa cells [28], FAM129A depletion increased expression of cell cycle inhibitors, such as p21, in PCa cells (Fig. 4). While senescence is induced in PCa cells (Fig. 4), apoptosis was instead induced in HeLa cells [17]. FAM129A thus contributes to the pro-survival role of ATF4 by suppressing senescence and apoptosis, depending on the environmental ques, or the cell type. Furthermore, FAM129A was recently identified as one of the genes that displayed an inverse regulation by ATF4 and p53 in response to mitochondrial respiration chain complex III inhibition [29], suggesting that it may be involved in mitochondrial homeostasis which is supported by ATF4 [30].

Importantly, our findings also reveal that FAM129A provides feedback regulation of the PERK-elF2 $\alpha$ pathway at multiple sites in PCa cells. elF2 $\alpha$ phosphorylation activates ATF4 expression that increases FAM129A levels which in turn inhibits elF2 $\alpha$ phosphorylation (Fig. 8). Concurrently, FAM129A regulates phosphorylation of other key proteins in protein translation, S6K1 and 4E-BP1 [17], thereby counteracting the inhibitory downstream effects of stress-induced p-elF2 $\alpha$ on translation. As both elF2 $\alpha$ phosphorylation and FAM129A expression are induced by stress, FAM129A provides negative feedback on the UPR to limit cell death.

Furthermore, FAM129A activates PERK phosphorylation, directly upstream of elF2 $\alpha$ (Fig. 7C). This is surprising since phosphorylation of the PERK target elF2 $\alpha$ is not similarly changed under the same conditions, which is at odds with our current understanding of the canonical PERK-elF2 $\alpha$ pathway [1, 2]. Even so, PERK is still involved in the FAM129A knockdown-mediated upregulation of elF2 $\alpha$ 
phosphorylation (Fig. 7B). In addition, we show that FAM129A and PERK physically interact (Fig. 7 and Fig. S7). The fact that FAM129A regulates PERK phosphorylation but does not have any sequence similarity to a kinase or phosphatase (or in fact any other known functional domain), suggests that it could function as a scaffold protein. FAM129A may thus regulate phosphorylation of its "target" proteins, such as PERK, by enabling or inhibiting interactions with other proteins, such as kinases and phosphatases. The function of this complex might be regulated by phosphorylation of FAM129A itself, as reported for the interaction between FAM129A and nucleophosmin [22]. In this context, the precise role of FAM129A in regulating PERK activity requires further investigation.

Regardless of the exact mechanism of action, FAM129A emerges as a key feedback regulator of the PERK-elF2 $\alpha$-ATF4 signaling in PCa cells where it acts as a rheostat to fine-tune the activity of this canonical pathway. In this model, depending on the level of FAM129A, PERK-eIF2 $\alpha$-ATF4 signaling may either be activated or inhibited. This could be similar to that previously described for MAPK signaling, whose activation can either promote proliferation or differentiation depending on the cellular environment [31].

\section{Future perspectives}

We do not know at present the exact molecular mechanisms that FAM129A employs to modulate the PERK-eIF2 $\alpha$ axis and enable increased prostate cancer growth. This information would be essential to potentially manipulate the PERK-eIF2a-ATF4-FAM129A axis to increase cellular stress and curb prostate cancer growth. The goal of targeting the stress response pathways in PCa is to induce signaling events that lead to cell death in the tumor without significantly affecting normal tissue. It is known that ATF4 can trigger both growth and apoptosis depending on the cellular context [5]. Thus, it may be more advantageous if one could identify ATF4 targets that are directly involved in growth and survival pathways. FAM129A fulfills this criterion as its targeting in a preclinical PCa model was remarkably effective to inhibit tumor growth, while the FAM129A knockout mice are healthy with only mild effects [17]. Alternatively, one could aim to specifically inhibit ATF4 in the tumor that could be achieved by siRNAs against ATF4 encapsulated in nanoparticles by systemic delivery. Targeted 
versions of these nanoparticles are desirable to increase efficacy and to limit potential side effects in future clinical applications for PCa. In addition, ATF4 and FAM129A can potentially synergize with other therapies currently used in the clinic which could open up for combinatorial targeting approaches. Our previous work has identified another canonical UPR signaling pathway, IRE1-XBP-1S, as being critical for PCa growth in vitro and in vivo $[9,32]$. Together with our current results, and the emerging importance of co-targeting of multiple pathways for successful anti-tumor activity (e.g. [33]; for a review, see [34]), it would be important to co-target both ATF4-FAM129A and IRE1-XBP-1S signaling to potentially achieve better tumor killing efficacy. Given the crosstalk of ATF4-FAM129A with the mTOR pathway, they could also constitute another pair for a combinatorial targeting approach. Additional work will be required to test these possibilities.

\section{Materials and Methods}

\section{Cell culture}

293T cells and the human PCa cell lines LNCaP, VCaP and 22Rv1 were purchased from the American Type Culture Collection (Rockville, MD) and cultured as described previously [35]. C4-2B cells were a gift from Dr. Lelund Chung. Where indicated, cells were treated with the ER stress inducers Thapsigargin (Tg), Tunicamycin (Tm), or Brefeldin A (Bfa) (Sigma-Aldrich, \#T9033, \#T7765, \#B6542). For experiments analyzing effects of ATF4 and FAM129A, cells were treated with 30nM Tg for 5h, unless stated otherwise. Detailed treatment information for other experiments is listed in supplemental methods.

\section{RNA interference}

Small interfering RNAs (siRNAs) were used to silence ATF4 (Dharmacon, \#L-005125), CEBPB (Qiagen, \#SI02777292+SI03058062+SI03022341+SI00073640), FAM129A (Qiagen, \#SI04167219+custom-made accordingly to [28]), PERK, GCN2, and PKR (Santa Cruz, \#36213, \#45644, \#36263). Allstar Negative 
Control siRNA (Qiagen, \#SI03650318) was used as a negative control. siRNA sequences and detailed method information are shown in supplemental methods.

Generation of inducible ATF4 knockdown and FAM129A overexpressing cells

pTRIPZ inducible short hairpin RNA (shRNA) vectors targeting ATF4 were purchased from Dharmacon (\#RHS4740-EG468). Scrambled control vector was generated from pTRIPZ by replacing the ATF4 shRNA sequence with that of GFP. FAM129A ORF clones were purchased from Dharmacon (\#MHS6278-202801471) and cloned into pTRIPZ. Lentivirus particles were produced in 293T cells using the Trans-Lentiviral ${ }^{\mathrm{TM}}$ shRNA Packaging Kit (Thermo Scientific, \#TLP5913) or Lipofectamine 3000 reagent (Invitrogen, \#L3000), respectively. LNCaP cells were transduced with the lentiviral particles. ATF4 knockdown or FAM129A overexpression was induced by doxycycline $(0.1-2.0 \mu \mathrm{g} / \mathrm{mL})$ (Sigma, \#D9891).

Ectopic expression of ATF4 and FAM129A

To obtain cells stably expressing ATF4, Precision lentiORF ATF4 and RFP vectors were purchased from Open Biosystems, Dharmacon (\#OHS5897-202616733, \#OHS5832). Virus was produced with TransLentiviral $^{\mathrm{TM}}$ Packaging kit (Thermo Fisher Scientific, \#TLP5919) and used for transduction of LNCaP cells. Ectopic expression of FAM129A was carried out by using an inducible FAM129A overexpressing cell line or by transfecting LNCaP cells with a plasmid vector specifying FAM129A-FLAG expression using Lipofectamine 3000 reagent.

\section{Cell proliferation assay}

Cells were cultured for the indicated times, trypsinized, stained with trypan blue, and counted using a hemocytometer. The data shown are representative of at least three independent experiments performed in triplicate.

\section{Colony formation assay}

Cells were trypsinized, seeded at a density of 5,000 cells per well in a 6-well plate, and cultured for 2-3 weeks. The cells were then fixed with methanol and stained with $0.4 \%$ crystal violet. Colonies 
were quantified by extracting crystal violet in $10 \%$ acetic acid and measuring the absorbance at $590 \mathrm{~nm}$. The data shown are representative of at least two independent experiments performed in triplicate.

Microarray analysis

LNCaP cells were transfected with control siRNA or an ATF4-specific siRNA, and 4 days later treated with $300 \mathrm{nM} \mathrm{Tg}$ for $5 \mathrm{~h}$. Total RNA was isolated as described previously [9]. Illumina Human HT-12 expression Bead-Chips (Illumina) were used for global transcriptome analysis according to the manufacturer's protocol. The experiment was performed in triplicates.

\section{Mass spectrometry}

LNCaP cells were transfected with control siRNA or an ATF4-specific siRNA, and 4 days later treated with either $300 \mathrm{nM}$ Tg for $5 \mathrm{~h}$ (MS-I) or 30nM Tg for $12 \mathrm{~h}$ (MS-II). Protein identification was performed by LC-MS after tryptic digestion. Proteins were identified using the protein identification software Mascot_v2.4.0 (Matrix Sciences) and mass spectra were searched against the Swiss-Prot database. The experiment was performed in triplicate.

\section{Quantitative PCR}

RNA extraction, cDNA synthesis, and quantitative PCR were performed as described previously [9]. Details are provided in Supplemental Methods. The data shown are representative of at least two independent experiments performed in triplicates.

\section{Western blot analysis}

Whole-cell extracts and Western analyses were performed by standard methods as described previously [9]. The data shown are representative of at least three independent experiments.

\section{Chromatin immunoprecipitation (ChIP)}

ChIP experiments were carried out according to a standard protocol (Upstate Biotechnology). Details are provided in Supplemental Methods The data shown are representative of at least two independent experiments performed in triplicates. 
Senescence-associated $\beta$-galactosidase staining

Senescence-associated $\beta$-galactosidase (SA- $\beta$-gal) activity was assessed using the SA- $\beta$-gal Staining Kit (Cell Signaling, \#9860) according to manufacturer's recommendations. At least 500 randomly selected cells were evaluated per sample and the percentage of positive cells is presented. The data shown are representative of two independent experiments performed in triplicates.

Co-immunoprecipitation (Co-IP)

293T cells were transfected with either FAM129A-FLAG and/or PERK-MYC plasmids (GeneScript, OHu10615C, OHu22427C) using Lipofectamine 3000 reagent. Cells were lysed in RIPA buffer and $500 \mu \mathrm{g}$ lysate was used for the IP with $2 \mu \mathrm{g}$ anti-FLAG (Cell Signaling Technology, 14793) and 50 $\mu \mathrm{L}$ of a 1:1 mixture of Surebeads ProteinA and ProteinG (BioRad, \#161-4013, \#161-4023). The eluted samples were analyzed by Western analysis using anti-FLAG (GeneScript, \#A00187) and anti-MYC (Cell Signaling Technology, \#2276). FAM129A and PERK mutants were generated by Q5 Site-Directed Mutagenesis Kit (NEB, \#E0554) or by restriction enzyme digestion (NEB), DNA fragment gel isolation (Zymogen) and Quick Ligation (NEB). The data shown are representative of at least two independent experiments.

Nanoliposomal siRNA targeting in PCa xenografts

In vivo therapeutic targeting of ATF4 and FAM129A by systemically administered nanoliposomal siRNA was carried out as described previously [11,35]. 6 animals were used per group. The animals were cage-wise allocated to experimental groups while the investigator was blinded to the group allocation during the whole experiment by pseudonymization of the siRNA identity. The experiments were performed in compliance with ethical regulations approved by the Norwegian Food Safety Authority.

Immunohistochemistry (IHC)

All radical prostatectomy specimens were obtained from Vancouver Prostate Centre Tissue bank with signed informed consent using a protocol approved by the Clinical Research Ethics Board of the 
University of British Columbia and the BC Cancer Agency. For ATF4, 3 tissue microarrays (TMAs) were used that were constructed from 298 patients, which 102 of those patients were subjected to neoadjuvant hormone therapy (NHT) prior to surgery. For FAM129A, 7 TMAs were stained that were constructed from 505 untreated specimens arrayed by their Gleason grade. The sets have complete clinical data with outcome information. IHC staining was conducted by Ventana autostainer model Discover $\mathrm{XT}^{\mathrm{TM}}$ (Ventana Medical System, Tuscan, Arizona) with enzyme labeled biotin streptavidin system and solvent resistant DAB Map kit using antibodies against ATF4 (Proteintech Group, \#108351-AP) and FAM129A (Signalway Antibody, \#21401).

In addition to general expression, luminal expression of FAM129A was determined. Following exclusion of 227 benign tumors and 9 patients who lack diagnosis or recurrence date annotations, remaining 463 patients with $(n=155)$ or without $(n=308)$ luminal FAM129A expression were investigated for their recurrence-free survival using IBM SPSS statistical software (v.25, IBM Corporation, NY, United States).

\section{Statistical analysis}

Mean and standard deviation values were calculated using Microsoft Excel software. The treatment effects in each experiment were compared by two-sided t-test. One-way analysis of variance (ANOVA) with a post hoc test was used for comparing multiple independent variables. Differences between groups were considered significant at $p<0.05$. All experiments were repeated three times. In animal experiments, we used 6 animals per group. For in vitro experiments, the results of one representative experiment are shown as mean values \pm SD or \pm SEM as indicated in the respective figure legends.

\section{Acknowledgements}

We would like to thank Veronica F. Blihovde, Xia Sheng and Deane Stryker for experimental help and members of the FS laboratory for helpful discussions. We would like to thank Jeffrey Petro for 
excellent technical support and Drs. Eva Corey and Colm Morrissey of University of Washington for providing LuCaP PDX lines. This work was supported by Norwegian Research Council grant 193337, Norwegian Cancer Society grant 419204, and Health South East Norway grant 36024. The microarray gene expression service was provided by the Genomics Core Facility (GCF), Norwegian University of Science and Technology (NTNU). GCF is funded by the Faculty of Medicine at NTNU and Central Norway Regional Health Authority. The LC-MS analyses were performed by the proteomics core facility at the Department of Biosciences, University of Oslo.

\section{Conflict of interest}

The authors declare that they have no conflict of interest.

\section{Author contributions}

$N P, M L$, and FS designed the study and experiments. NP and ML performed and analyzed most of the experiments. MT conducted ChIP experiments. HZN performed IP-Western experiments. EA and JS performed colocalization studies. YiJ conducted nanoliposomal siRNA delivery experiments. DK contributed to growth assays. PB and AU conducted PDX-derived organoid experiments. LF and PR provided tissue microarrays of $\mathrm{PCa}$, performed IHC analyses, and their interpretation. $\mathrm{BO}, \mathrm{NK}$, and HMM provided nanoliposomal siRNAs. YaJ performed IHC analyses on consecutive slides and helped with supervision. OFK did statistical analysis of IHC data. MP scored TMAs. HD provided expertise in IHC interpretation. FS supervised the project. NP, ML, and FS wrote the manuscript.

\section{Data Availability}

Supplementary information is available at Oncogene's website. The microarray data discussed in this publication have been deposited in NCBI's Gene Expression Omnibus [36] and are accessible through 
GEO

Series

accession

number

GSE125826

(http://www.ncbi.nlm.nih.gov/geo/query/acc.cgi?acc=GSE125826).

The mass spectrometry proteomics data discussed in this publication have been deposited to the ProteomeXchange Consortium (http://proteomecentral.proteomexchange.org) via the PRIDE partner repository [37].

\section{References}

1 Clarke HJ, Chambers JE, Liniker E, Marciniak SJ. Endoplasmic reticulum stress in malignancy. Cancer Cell 2014; 25: 563-573.

2 Wang M, Kaufman RJ. The impact of the endoplasmic reticulum protein-folding environment on cancer development. Nat Rev Cancer 2014; 14: 581-597.

3 Chevet E, Hetz C, Samali A. Endoplasmic reticulum stress-activated cell reprogramming in oncogenesis. Cancer Discov 2015; 5: 586-597.

$4 \quad$ Ameri K, Harris AL. Activating transcription factor 4. Int J Biochem Cell Biol 2008; 40: 14-21.

5 Wortel IMN, van der Meer LT, Kilberg MS, van Leeuwen FN. Surviving Stress: Modulation of ATF4-Mediated Stress Responses in Normal and Malignant Cells. Trends Endocrinol Metab 2017; 28: 794-806.

6 Han J, Back SH, Hur J, Lin YH, Gildersleeve R, Shan J et al. ER-stress-induced transcriptional regulation increases protein synthesis leading to cell death. Nat Cell Biol 2013; 15: 481-490.

7 Attard G, Parker C, Eeles RA, Schröder F, Tomlins SA, Tannock I et al. Prostate cancer. Lancet 2016; 387: 70-82.

8 Storm M, Sheng X, Arnoldussen YJ, Saatcioglu F. Prostate cancer and the unfolded protein response. Oncotarget 2016; 7: 54051-54066.

9 Sheng X, Arnoldussen YJ, Storm M, Tesikova M, Nenseth HZ, Zhao S et al. Divergent androgen regulation of unfolded protein response pathways drives prostate cancer. EMBO Mol Med 2015; 7: 788-801.

10 Ye J, Kumanova M, Hart LS, Sloane K, Zhang H, De Panis DN et al. The GCN2-ATF4 pathway is critical for tumour cell survival and proliferation in response to nutrient deprivation. EMBO J 2010; 29: 2082-2096.

11 Jin Y, Qu S, Tesikova M, Wang L, Kristian A, Maelandsmo GM et al. Molecular circuit involving KLK4 integrates androgen and mTOR signaling in prostate cancer. Proc Natl Acad Sci U S A 2013; 110: E2572-2581. 
12 Mills IG. Maintaining and reprogramming genomic androgen receptor activity in prostate cancer. Nat Rev Cancer 2014; 14: 187-198.

13 Shan J, Zhang F, Sharkey J, Tang TA, Ord T, Kilberg MS. The C/ebp-Atf response element (CARE) location reveals two distinct Atf4-dependent, elongation-mediated mechanisms for transcriptional induction of aminoacyl-tRNA synthetase genes in response to amino acid limitation. Nucleic Acids Res 2016; 44: 9719-9732.

14 Kilberg MS, Balasubramanian M, Fu L, Shan J. The transcription factor network associated with the amino acid response in mammalian cells. Adv Nutr 2012; 3: 295-306.

15 Yu K, Mo D, Wu M, Chen H, Chen L, Li M et al. Activating transcription factor 4 regulates adipocyte differentiation via altering the coordinate expression of CCATT/enhancer binding protein beta and peroxisome proliferator-activated receptor gamma. FEBS J 2014; 281: 23992409.

16 Cohen DM, Won KJ, Nguyen N, Lazar MA, Chen CS, Steger DJ. ATF4 licenses C/EBPbeta activity in human mesenchymal stem cells primed for adipogenesis. Elife 2015; 4: e06821.

17 Sun GD, Kobayashi T, Abe M, Tada N, Adachi H, Shiota A et al. The endoplasmic reticulum stress-inducible protein Niban regulates elF2alpha and S6K1/4E-BP1 phosphorylation. Biochem Biophys Res Commun 2007; 360: 181-187.

18 Shaw GL, Whitaker H, Corcoran M, Dunning MJ, Luxton H, Kay J et al. The Early Effects of Rapid Androgen Deprivation on Human Prostate Cancer. Eur Urol 2016; 70: 214-218.

19 Massie CE, Lynch A, Ramos-Montoya A, Boren J, Stark R, Fazli L et al. The androgen receptor fuels prostate cancer by regulating central metabolism and biosynthesis. EMBO J 2011; 30: 2719-2733.

20 Thomas BC, Kay JD, Menon S, Vowler SL, Dawson SN, Bucklow $L$ et al. Whole blood mRNA in prostate cancer reveals a four-gene androgen regulated panel. Endocr Relat Cancer 2016; 23: 797-812.

21 Halterman MW, De Jesus C, Rempe DA, Schor NF, Federoff HJ. Loss of c/EBP-beta activity promotes the adaptive to apoptotic switch in hypoxic cortical neurons. Mol Cell Neurosci 2008; 38: 125-137.

22 Ji H, Ding Z, Hawke D, Xing D, Jiang BH, Mills GB et al. AKT-dependent phosphorylation of Niban regulates nucleophosmin- and MDM2-mediated p53 stability and cell apoptosis. EMBO Rep 2012; 13: 554-560.

23 Adachi H, Majima S, Kon S, Kobayashi T, Kajino K, Mitani $\mathrm{H}$ et al. Niban gene is commonly expressed in the renal tumors: a new candidate marker for renal carcinogenesis. Oncogene 2004; 23: 3495-3500.

24 Ito S, Fujii H, Matsumoto T, Abe M, Ikeda K, Hino O. Frequent expression of Niban in head and neck squamous cell carcinoma and squamous dysplasia. Head Neck 2010; 32: 96-103.

25 Kannangai R, Diehl AM, Sicklick J, Rojkind M, Thomas D, Torbenson M. Hepatic angiomyolipoma and hepatic stellate cells share a similar gene expression profile. Hum Pathol 2005; 36: 341-347. 
Pakos-Zebrucka K, Koryga I, Mnich K, Ljujic M, Samali A, Gorman AM. The integrated stress response. EMBO Rep 2016; 17: 1374-1395.

27 Harding HP, Zhang Y, Zeng H, Novoa I, Lu PD, Calfon M et al. An integrated stress response regulates amino acid metabolism and resistance to oxidative stress. Mol Cell 2003; 11: 619633.

28 Yuki R, Aoyama K, Kubota S, Yamaguchi N, Kubota S, Hasegawa $\mathrm{H}$ et al. Overexpression of zinc-finger protein 777 (ZNF777) inhibits proliferation at low cell density through downregulation of FAM129A. J Cell Biochem 2015; 116: 954-968.

29 Evstafieva AG, Kovaleva IE, Shoshinova MS, Budanov AV, Chumakov PM. Implication of KRT16, FAM129A and HKDC1 genes as ATF4 regulated components of the integrated stress response. PLoS One 2018; 13: e0191107.

30 Quirós PM, Prado MA, Zamboni N, D'Amico D, Williams RW, Finley D et al. Multi-omics analysis identifies ATF4 as a key regulator of the mitochondrial stress response in mammals. J Cell Biol 2017; 216: 2027-2045.

31 Marshall CJ. Specificity of receptor tyrosine kinase signaling: transient versus sustained extracellular signal-regulated kinase activation. Cell 1995; 80: 179-185.

32 Sheng $\mathrm{X}$, Nenseth $\mathrm{HZ}$, Qu S, Kuzu OF, Frahnow T, Simon L et al. IRE1 $\alpha-X B P 1 s$ pathway promotes prostate cancer by activating c-MYC signaling. Nat Commun 2019; 10: 323.

33 Brijwani N, Jain M, Dhandapani M, Zahed F, Mukhopadhyay P, Biswas M et al. Rationally cotargeting divergent pathways in KRAS wild-type colorectal cancers by CANscript technology reveals tumor dependence on Notch and Erbb2. Sci Rep 2017; 7: 1502.

34 Nevedomskaya E, Baumgart SJ, Haendler B. Recent Advances in Prostate Cancer Treatment and Drug Discovery. Int J Mol Sci 2018; 19.

35 Jin Y, Wang L, Qu S, Sheng X, Kristian A, Maelandsmo GM et al. STAMP2 increases oxidative stress and is critical for prostate cancer. EMBO Mol Med 2015; 7: 315-331.

36 Edgar R, Domrachev M, Lash AE. Gene Expression Omnibus: NCBI gene expression and hybridization array data repository. Nucleic Acids Res 2002; 30: 207-210.

37 Vizcaíno JA, Côté RG, Csordas A, Dianes JA, Fabregat A, Foster JM et al. The PRoteomics IDEntifications (PRIDE) database and associated tools: status in 2013. Nucleic Acids Res 2013; 41: D1063-1069. 


\section{Figure Legends}

Figure 1. ATF4 promotes PCa cell growth in vitro and in vivo and its expression is upregulated in human PCa specimens.

A) LNCaP cells were transfected with either control siRNA (siCtrl) or two independent ATF4specific siRNAs (siATF4-1 and -2). ATF4 expression was assessed by qPCR and Western analysis upon Tg stimulation (300nM). ${ }^{*} p<0,0001$.

B) ATF4 knockdown decreases PCa cell growth. LNCaP cells transfected with either control siRNA or two independent ATF4-specific siRNAs were cultured for the indicated times and cell numbers were determined by trypan blue staining. ${ }^{*} p<0.05$.

C) ATF4 knockdown decreases colony formation. LNCaP cells were transfected with either control siRNA or two independent ATF4-specific siRNAs and cultured for three weeks. Colonies formed were stained and quantified as described in Materials and Methods. ${ }^{*} p<$ 0.05 .

D) ATF4 knockdown decreases cell cycle related gene expression. LNCaP cells transfected with either control siRNA or two independent ATF4-specific siRNAs were synchronized for $48 \mathrm{~h}$ and then released into the cell cycle for 24h. Expression of cell cycle related genes was analyzed by Western analysis upon $\mathrm{Tg}$ stimulation.

E) ATF4 knockdown increases apoptosis sensitivity of PCa cells. LNCaP cells transfected as described in C were treated with TRAIL and LY294002 for 6h. Cleaved PARP and cleaved Caspase 3 levels were determined by Western analysis.

F) Therapeutic targeting of ATF4 by systemically injected nanoliposomal ATF4-specific siRNA dramatically inhibits growth of VCaP-derived xenograft tumors in vivo. VCaP cells were implanted subcutaneously into athymic male nude mice. Once tumors were visible, mice $(n=$ 6 per group) were given either empty nanoliposomes or ATF4-specific siRNA as described in Materials and Methods. Tumor volumes were measured at the indicated time points. Error bars indicate SEM. ${ }^{*} p<0,05$.

G) Immunohistochemistry was used to assess ATF4 expression in normal and malignant human prostate specimens. Tissue microarrays (TMAs) with normal prostate $(n=90)$, hormone naïve prostate tumors $(n=316)$ and neoadjuvant hormone treated $(N H T)$ tumors $(n=130)$ were analyzed by immunohistochemistry as described in Materials and Methods. Representative images are shown.

H) Quantification of ATF4 staining intensity in TMAs described in A is shown. Error bars indicate SEM. ${ }^{*} p<0.000001$. 
Figure 2. Global transcriptomic and proteomic gene expression analysis of ATF4 targets during ER stress.

A) Flowchart describing the methods used to identify novel ATF4 target genes in PCa cells.

B) LNCaP cells were transfected with control or an ATF4-specific siRNA and subjected to global transcriptomic (MA) and proteomic (MS-I and MS-II)) gene expression analysis. The Venn diagram depicts the number of overlapping downregulated genes/proteins between the three different arrays. MA and MS-I analyses were performed after $5 \mathrm{~h}$ of Tg treatment, while MS-II analysis was after $12 \mathrm{~h}$ of Tg treatment.

C) ATF4 re-expression rescues gene expression related to amino acid and tRNA enzymes and transporters. PSAT1, SLC1A5, GARS and XPO5 are shown as examples per group. LNCaP cells stably expressing the open reading frame (ORF) of ATF4 or Red Fluorescent Protein (RFP) as control were transfected with either control siRNA or two independent siRNAs targeting the ORF (siATF4-1) or the 5'-UTR (siATF4-2) of the ATF4 mRNA and gene expression was analyzed by qPCR upon Tg stimulation. ${ }^{*} \mathrm{p}<0,01$.

D) ATF4 knockdown decreases CEBPB expression. LNCaP cells were transfected with either control siRNA or two independent ATF4-specific siRNAs, treated with $\operatorname{Tg}(300 \mathrm{nM})$, and CEBPB expression was analyzed by $q P C R .{ }^{*} p<0,001$.

E) ChIP analysis of ATF4 binding to the CEBPB gene. LNCaP cells were transfected with either control siRNA or an ATF4-specific siRNA, treated with vehicle or $\mathrm{Tg}$, and ChIP assay was performed as described in Materials and Methods using an ATF4-specific antibody. ${ }^{*} \mathrm{p}<$ 0,000001 .

Figure 3. FAM129A is a novel ATF4 target gene that is induced in PCa cells upon ER stress.

A) FAM129A expression is increased upon ER stress. LNCaP cells were treated with 100nM Tg, $5 \mu \mathrm{g} / \mathrm{mL}$ Tunicamycin (Tm) or 50 $\mu \mathrm{M}$ Brefeldin A (Bfa) for up to 8h and FAM129A expression was analyzed by qPCR. ${ }^{*} p<0,01$.

B) ATF4 knockdown decreases FAM129A expression. LNCaP cells were transfected with either control siRNA or two independent ATF4-specific siRNAs, treated with Tg, and FAM129A expression was analyzed by qPCR after $5 \mathrm{~h}$ of $\mathrm{Tg}$ induction (300nM) and by Western analysis after $12 \mathrm{~h}$ of Tg induction (30nM). * $\mathrm{p}<0,0001$.

C) Downregulation of FAM129A upon ATF4 knockdown is rescued by ATF4 re-expression. LNCaP cells stably expressing the ORF of ATF4 or RFP were transfected with either control siRNA or two independent siRNAs targeting the ORF (siATF4-1) or the 5'-UTR (siATF4-2) of the ATF4 gene and FAM129A expression was analyzed by qPCR upon Tg stimulation. LN-ATF4 cells 
were protected against ATF4 knockdown mediated downregulation of FAM129A by siATF4-2, but not by siATF4-1. ${ }^{*} p<0,01$.

D) Same experiment as in B, but protein levels were determined by Western analysis. R: LN-RFP cells, A: LN-ATF4 cells.

E) ChIP analysis shows ATF4 binding to a CARE sequence in the intronic region of the FAM129A gene. LNCaP cells were transfected with either control siRNA or an ATF4-specific siRNA, treated with vehicle or Tg, fixed, and ChIP assay was performed as described in Materials and Methods using an ATF4-specific antibody. ${ }^{*} \mathrm{p}<0,000001$.

F) Schematic overview and details of the CARE sequence within a C/EBP $\beta$ binding site in the human FAM129A gene.

Figure 4. FAM129A depletion inhibits cell growth and induces senescence in PCa cells.

A) LNCaP cells were transfected with either control siRNA or two independent FAM129Aspecific siRNAs (siFAM-1 and -2). FAM129A expression was assessed by qPCR and Western analysis upon Tg stimulation. ${ }^{*} p<0,01$.

B) FAM129A knockdown decreases PCa cell growth. LNCaP cells transfected with either control siRNA or two independent FAM129A-specific siRNAs were cultured for the indicated times, and cell numbers were determined by trypan blue staining. ${ }^{*} p<0.05$.

C) FAM129A knockdown decreases colony formation. LNCaP cells were transfected with either control siRNA or two independent FAM129A-specific siRNAs and cultured for three weeks. Colonies formed were stained and quantified as described in Materials and Methods. ${ }^{*} p<$ 0,01 .

D) FAM129A knockdown decreases expression of proliferative markers. LNCaP cells transfected with either control siRNA or two independent FAM129A-specific siRNAs were synchronized by starvation for $48 \mathrm{~h}$ and then released into the cell cycle for $24 \mathrm{~h}$. Expression of Ki67 and PCNA mRNA levels was analyzed by qPCR 6 days after transfection upon Tg stimulation. ${ }^{*} p<$ 0,001 .

E) FAM129A knockdown does not significantly affect apoptosis sensitivity. LNCaP cells transfected as described in A were treated with TRAIL for 12 h, 6 days after. Cleaved PARP and cleaved Caspase 3 levels were determined by Western analysis.

F) Expression of $G 2$ and $M$ phase-driving cyclins and E2Fs is reduced upon FAM129A knockdown. LNCaP cells transfected with either control siRNA or two independent FAM129Aspecific siRNAs were synchronized by starvation for $48 \mathrm{~h}$ and then released into the cell cycle 
for 24 h. Expression of cyclin A2, B1, B2, E2F1 and E2F2 was analyzed 6 days after transfection upon Tg stimulation. ${ }^{*} \mathrm{p}<0,01$.

G) FAM129A knockdown decreases Rb phosphorylation. LNCaP cells were transfected with either control siRNA or two independent FAM129A-specific siRNAs. Six days later, $\mathrm{Rb}$ phosphorylation was analyzed by Western analysis upon $\mathrm{Tg}$ stimulation.

H) FAM129A depletion increases expression of cell cycle inhibitors. LNCaP cells were transfected with either control siRNA or two independent FAM129A-specific siRNAs. Six days later of $p 21 p 15$ expression was analyzed by qPCR. ${ }^{*} p<0,001$.

I) FAM129A knockdown affects cell morphology. LNCaP cells treated as described in G were stained with hematoxylin-eosin and analyzed under the microscope. Red arrowheads mark cells showing a change in morphology.

J) FAM129A depletion increases SAßGal activity. LNCaP cells treated as described in G were fixed, SAßGal assay was performed and positive cells quantified. ${ }^{*} p<0,0000001$.

Figure 5. Targeting FAM129A in a preclinical PCa model inhibits tumor growth and its expression is increased in human PCa specimens.

A) In vivo therapeutic targeting of FAM129 by nanoliposomal FAM129A-specific siRNA dramatically blocks growth of VCaP-derived tumors in vivo. VCaP cells were implanted subcutaneously into athymic male nude mice. Once tumors were visible, mice $(n=6$ per group) were given empty nanoliposomes (same experiment as in Fig. 1F) or FAM129Aspecific siRNA as described in Materials and Methods. Tumor volumes were measured the indicated time points. Error bars indicate SEM. ${ }^{*} p<0,05$.

B) Androgen treatment induces FAM129A expression. LNCaP cells were treated with 1nM R1881 for the indicated times and FAM129A expression was determined by Western analysis.

C) Immunohistochemistry was used to assess FAM129A expression in normal and malignant human prostate specimens. TMAs with normal prostate $(n=219)$ and prostate tumors $(n=$ 863) were analyzed by IHC as described in Materials and Methods. Representative images are shown.

D) Quantification of FAM129A staining in TMAs described in C is shown. The graph depicts the total score, the multiplication product of staining intensity and percentage of stained cells. Error bars indicate SEM. ${ }^{*} p=8,77393 \mathrm{E}-16$.

E) Representative images showing stronger FAM129A staining by IHC in hyperplastic areas of prostate tumors described in C. 
F) Kaplan-Meier estimates of recurrence free survival of 463 PCa patients bearing tumors with or without luminal FAM129A expression. P values represent Mantel-Cox test of equality of survival distributions for the two groups.

Figure 6. ATF4 and FAM129 knockdown significantly inhibit PCa PDX organoid viability.

A) LuCaP PDX organoids were grown on Nunclon Sphera ultra low attachment 6-well plates and transfected with 100nM of control, ATF4 or FAM129A siRNAs for 7 days. Live and dead cells were labeled with fluorescent reagents calcein-AM and ethidium homodimer-1, respectively. A robust increase in cell death was observed after ATF4 and FAM129A siRNA treatment compared to control siRNA. LuCaP 23.1 is shown as an example.

B) Fluorescent images of dead cells were quantitated and mean values $(-/+\mathrm{SE})$ were presented in bar graphs. Groups were compared by Student's T test.

Figure 7. FAM129A differentially affects PERK and elF2 $\alpha$ activities.

A) FAM129A knockdown affects phosphorylation of proteins involved in translation. LNCaP cells were transfected either with control siRNA or two independent FAM129A-specific siRNAs. Six days later 4E-BP1 and elF2 $\alpha$ phosphorylation, as well as ATF4 and CHOP expression, were analyzed by Western analysis upon a $5 \mathrm{~h}$ Tg stimulation.

B) PERK is involved in FAM129A depletion-mediated phosphorylation of elF2 $\alpha$. LNCaP cells were transfected with either control, PERK- (siPERK), GCN2- (siGCN2) or PKR-specific (siPKR) siRNA alone or in combination with FAM129A-specific siRNA. 3 days later, knockdown of the respective proteins was confirmed and elF $2 \alpha$ phosphorylation was analyzed by western analysis.

C) FAM129A increases p-PERK levels. LNCaP cells were transfected with the indicated amounts of a FAM129A expression vector. After six days, the expression of FAM129A, p-PERK, and pelF2 $\alpha$ was analyzed by Western analysis upon a $5 \mathrm{~h} \mathrm{Tg}$ treatment.

D) FAM129A knockdown decreased Tg-induced p-PERK levels. Expression and phosphorylation of PERK in LNCaP cells treated as described in A was analyzed by Western analysis 6 days after transfection.

E) FAM129A interacts with PERK. A MYC-tagged PERK expression vector (PERK-MYC) was transiently expressed in 293T cells with a FLAG-tagged FAM129A expression vector (FAM129A-FLAG). Immunoprecipitation upon Tg treatment was performed with a FLAGspecific antiserum as described in Materials and Methods. The lysates and 
immunoprecipitates were then subjected to Western analysis with MYC- and FLAG-specific antiserum.

F) Schematic depiction of the FAM129A mutants used for IP experiments. The numbers indicate the amino acid residues which were deleted in the mutants. F=FLAG tag.

G) Schematic depiction of the PERK mutant used for IP experiments. M= MYC tag, TM= transmembrane domain, Sensor= ER sensor domain, Kinase= kinase domain.

H) Determination of the domains involved in FAM129A and PERK interactions. PERK-MYC full length or deletion mutant constructs were transiently expressed in 293T cells together with FAM129A-FLAG full length or the deletion mutants as indicated. Immunoprecipitations were performed with a FLAG-specific antiserum which were then subjected to Western analysis with a MYC-specific antibody.

I) FAM129A colocalizes with PERK. LNCaP cells ectopically expressing FAM129A-FLAG (FAM129A-F) and PERK-MYC (PERK-M) were examined by confocal immunofluorescence microscopy. Representative images are shown. The scale bar is $5 \mu \mathrm{m}$.

J) Quantification of FAM129A-PERK colocalization determined by the Pearson's correlation method.

Figure 8. Model for the role of ATF4-FAM129A signaling on PERK pathway in PCa cells.

Once its expression is activated by ER stress, ATF4 induces FAM129A expression by direct binding to its promoter. FAM129A then inhibits senescence/apoptosis. In addition, through both positive and negative feedback loops, it regulates the PERK-eIF2 $\alpha$ pathway that would allow the cell to fine tune its response to incoming stress depending on the context. 
A

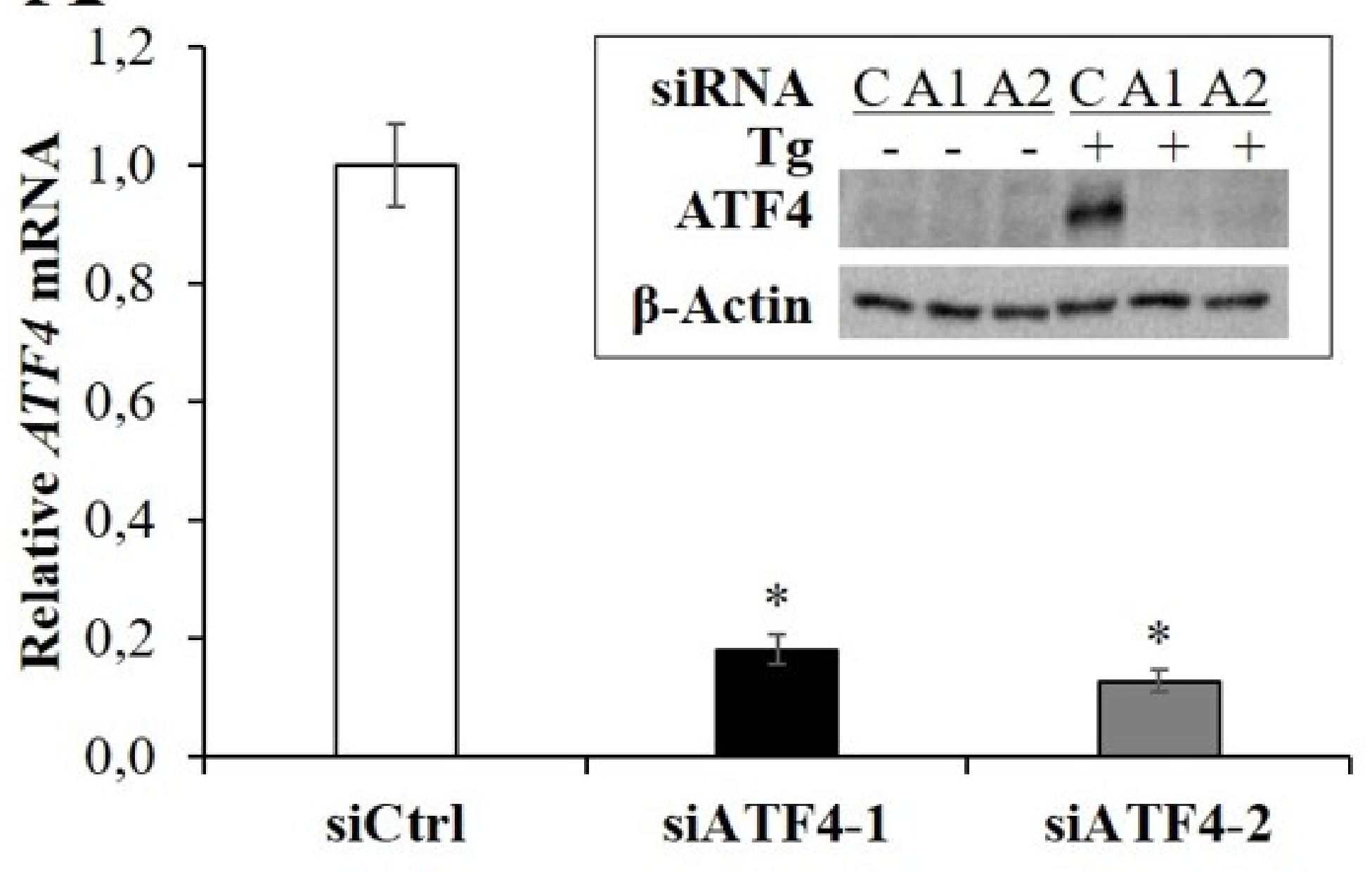

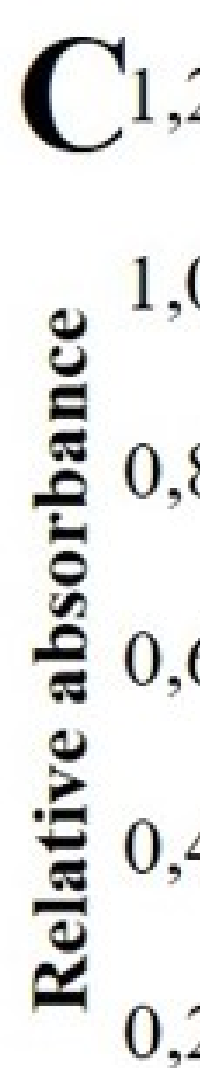

0,0

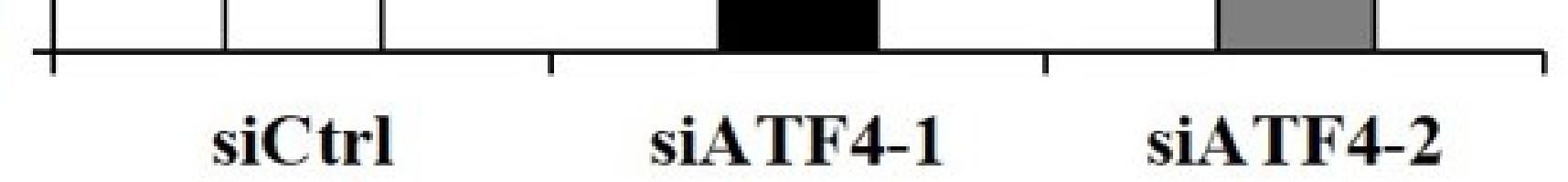

B

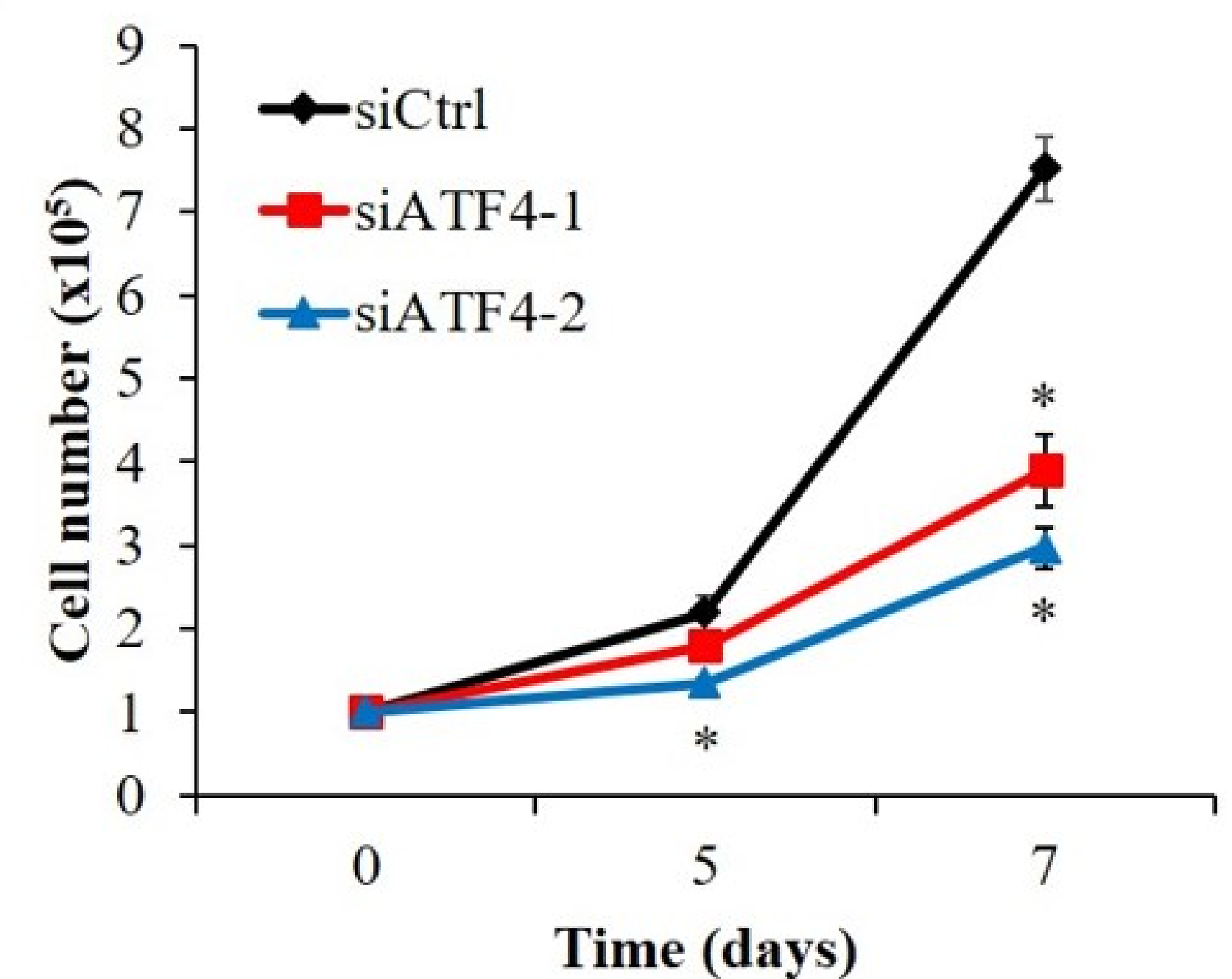

D

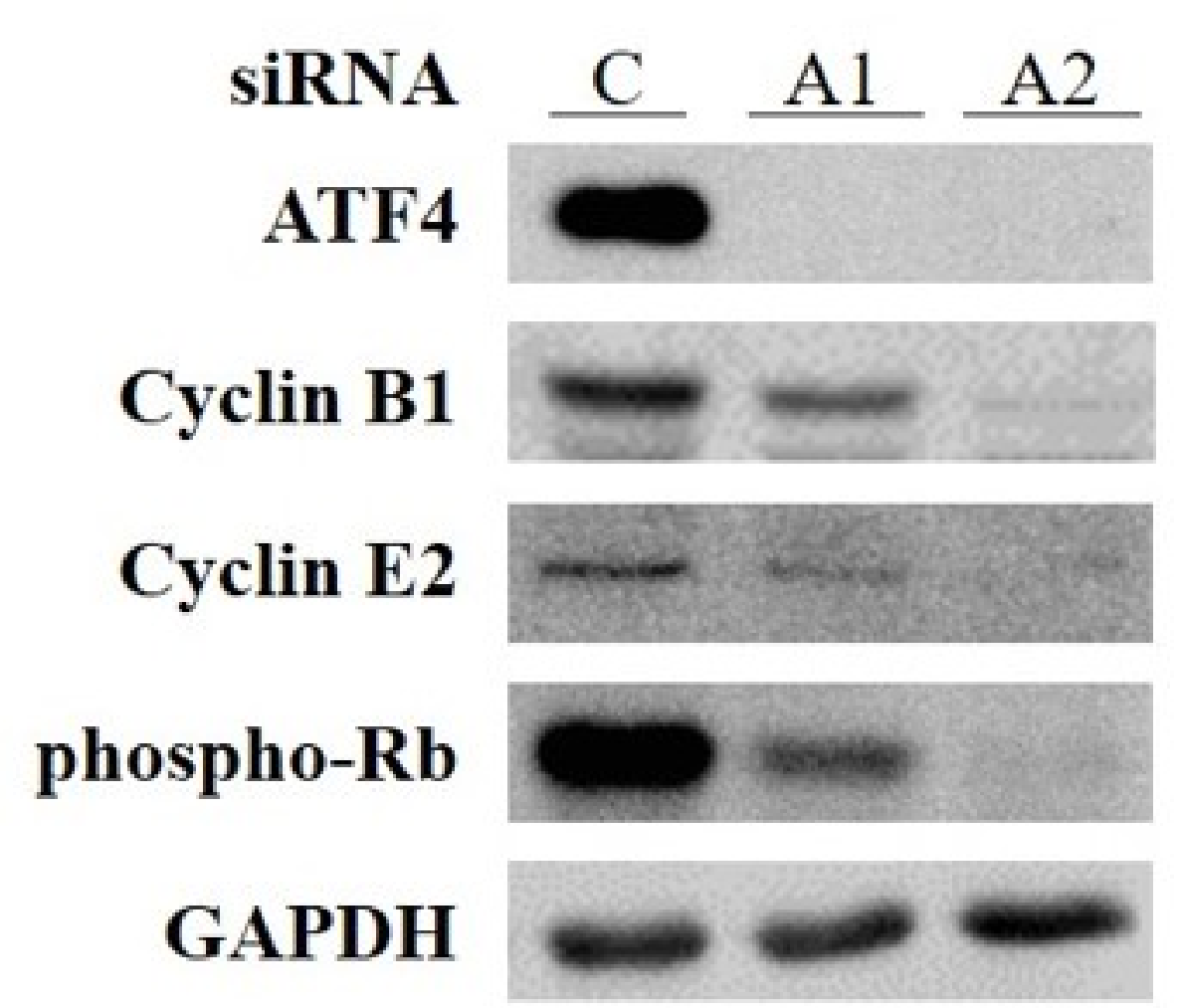

$\mathbf{E}$

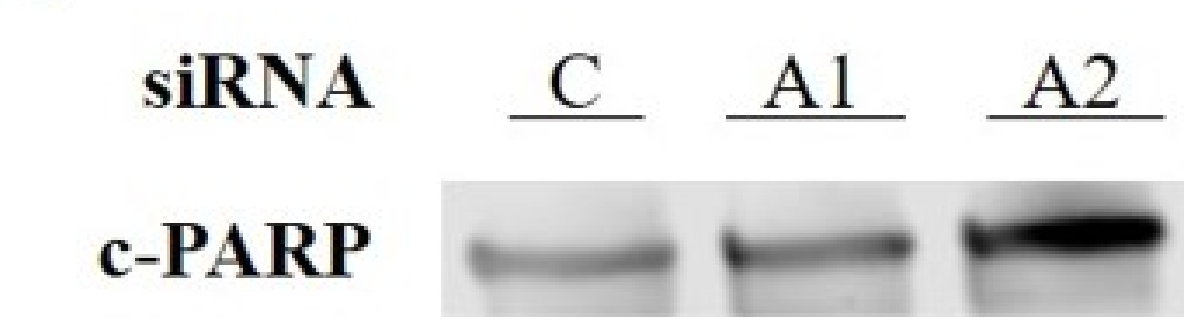

c-Caspase 3

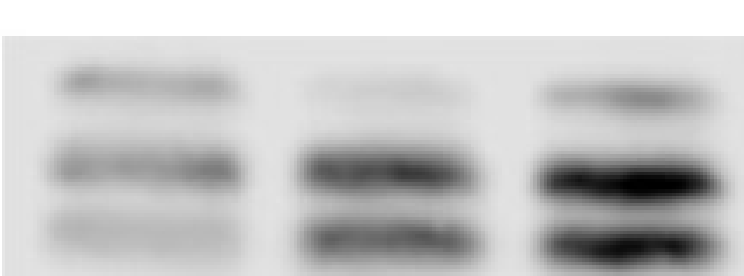

GAPDH
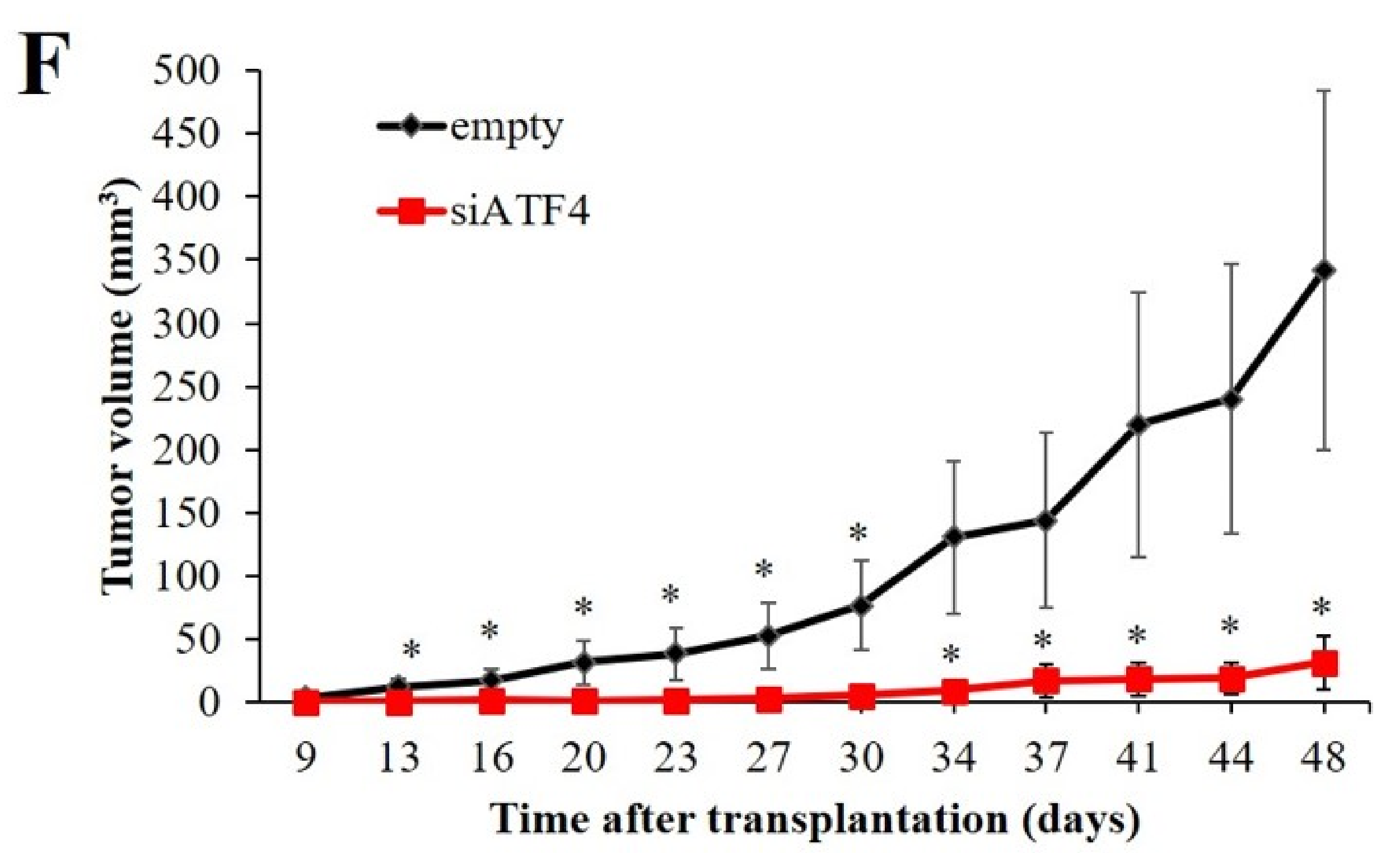

\section{G}

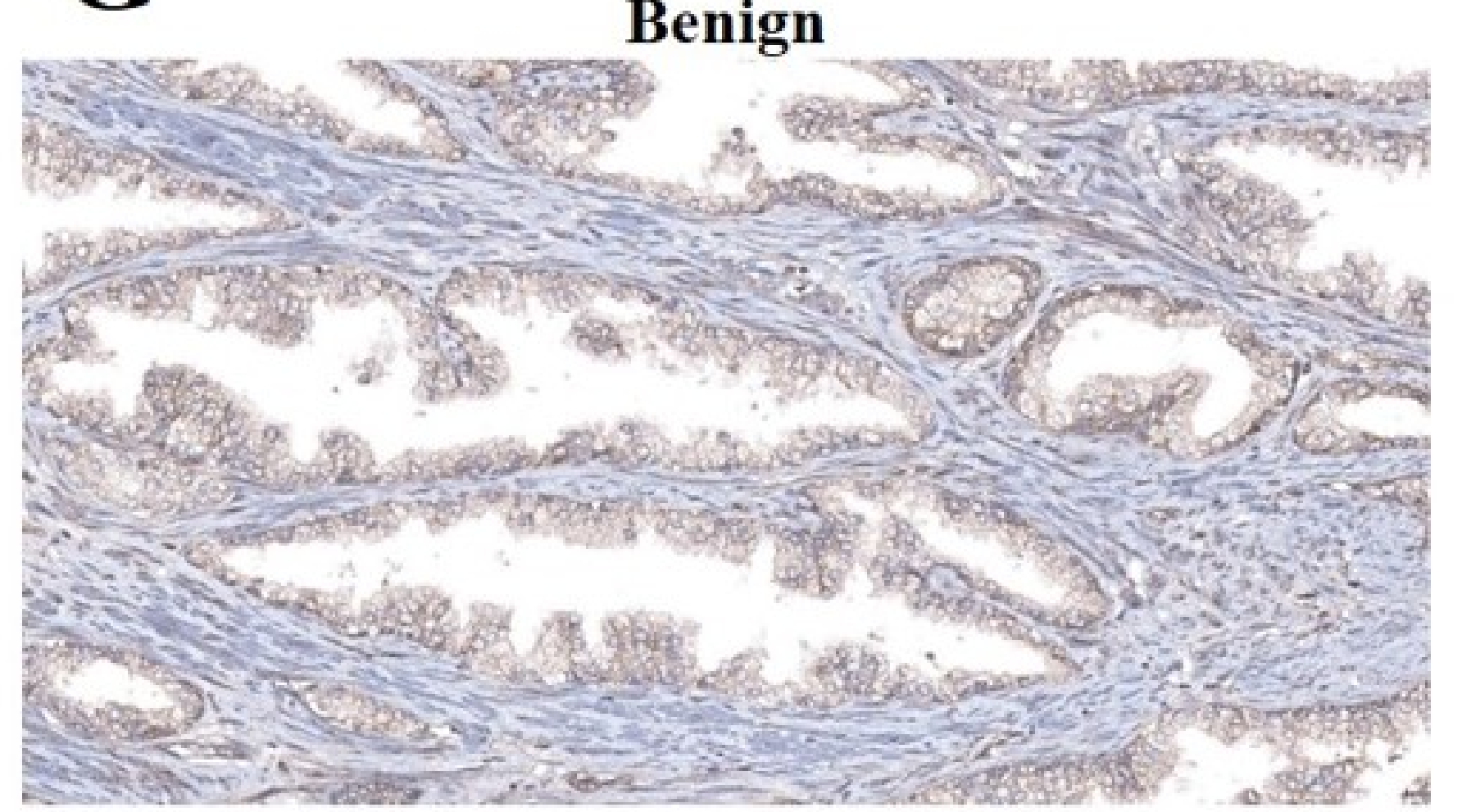

NHT-treated
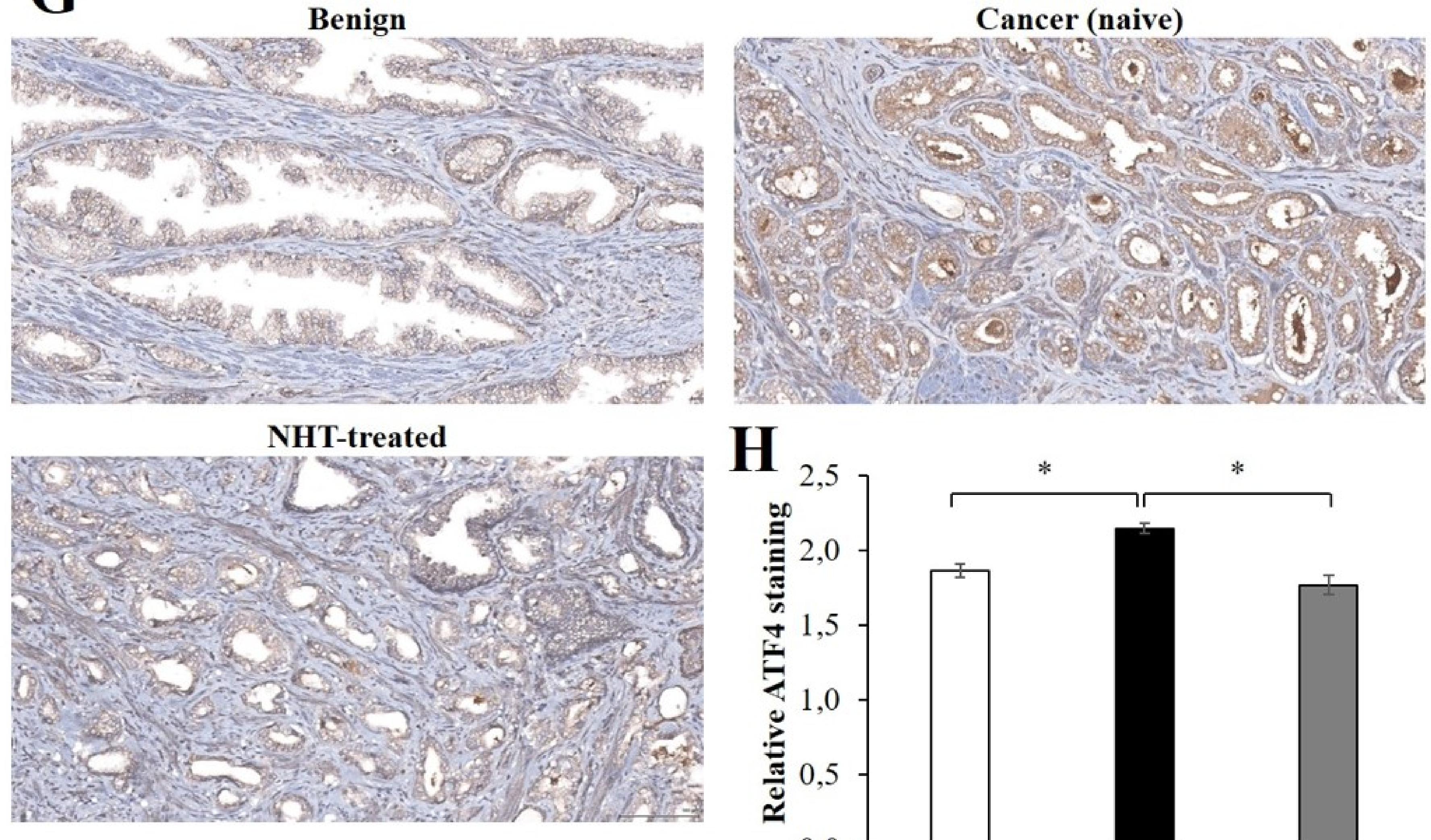

H

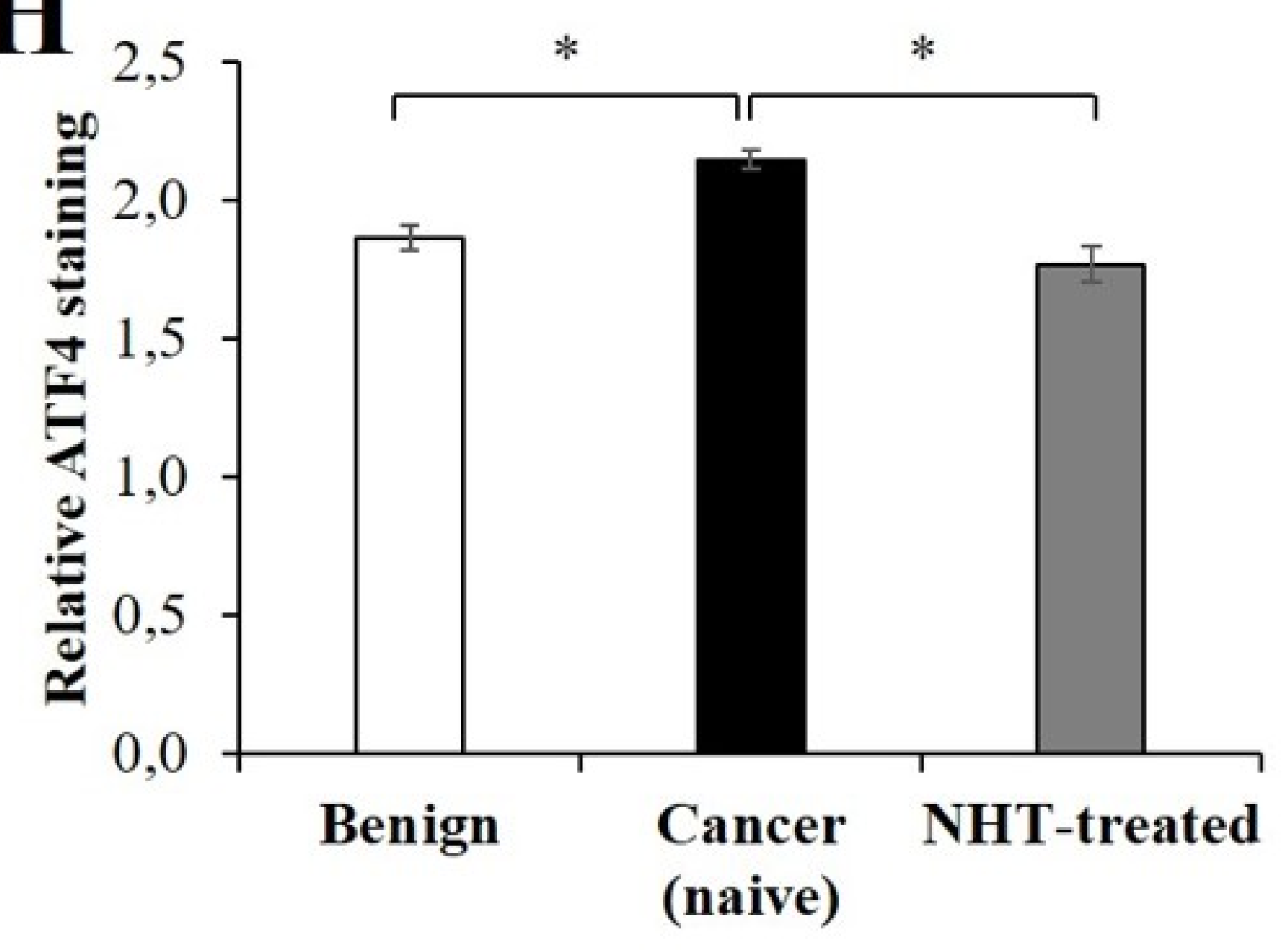


knockdown

Transcriptome Proteome analysis (MA) analysis (MS)

Possible ATF4 target genes

Rescue experiment

ChIP experiment

Novel ATF4 target gene
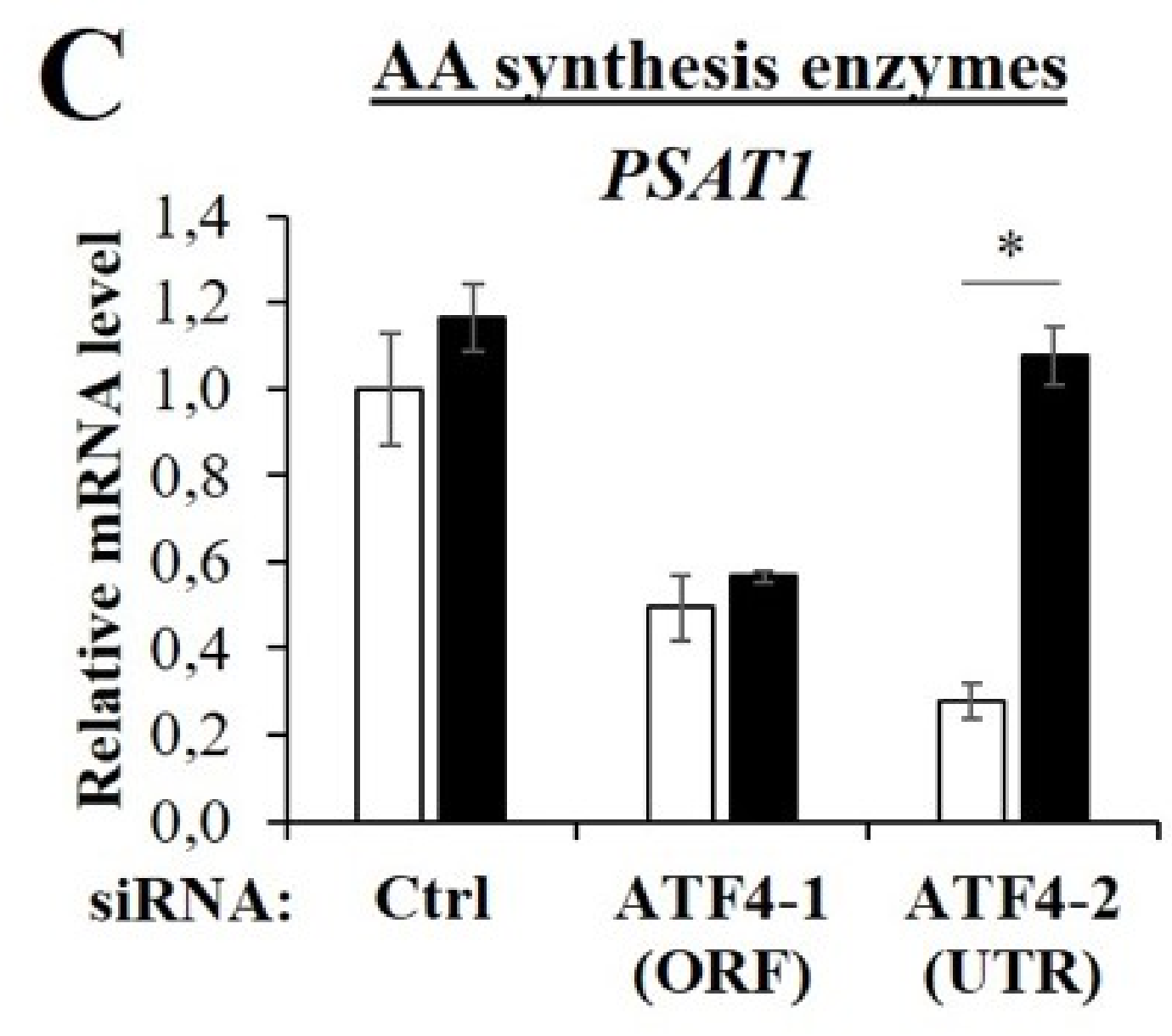

$\underline{\text { tRNA ligases }}$

GARS

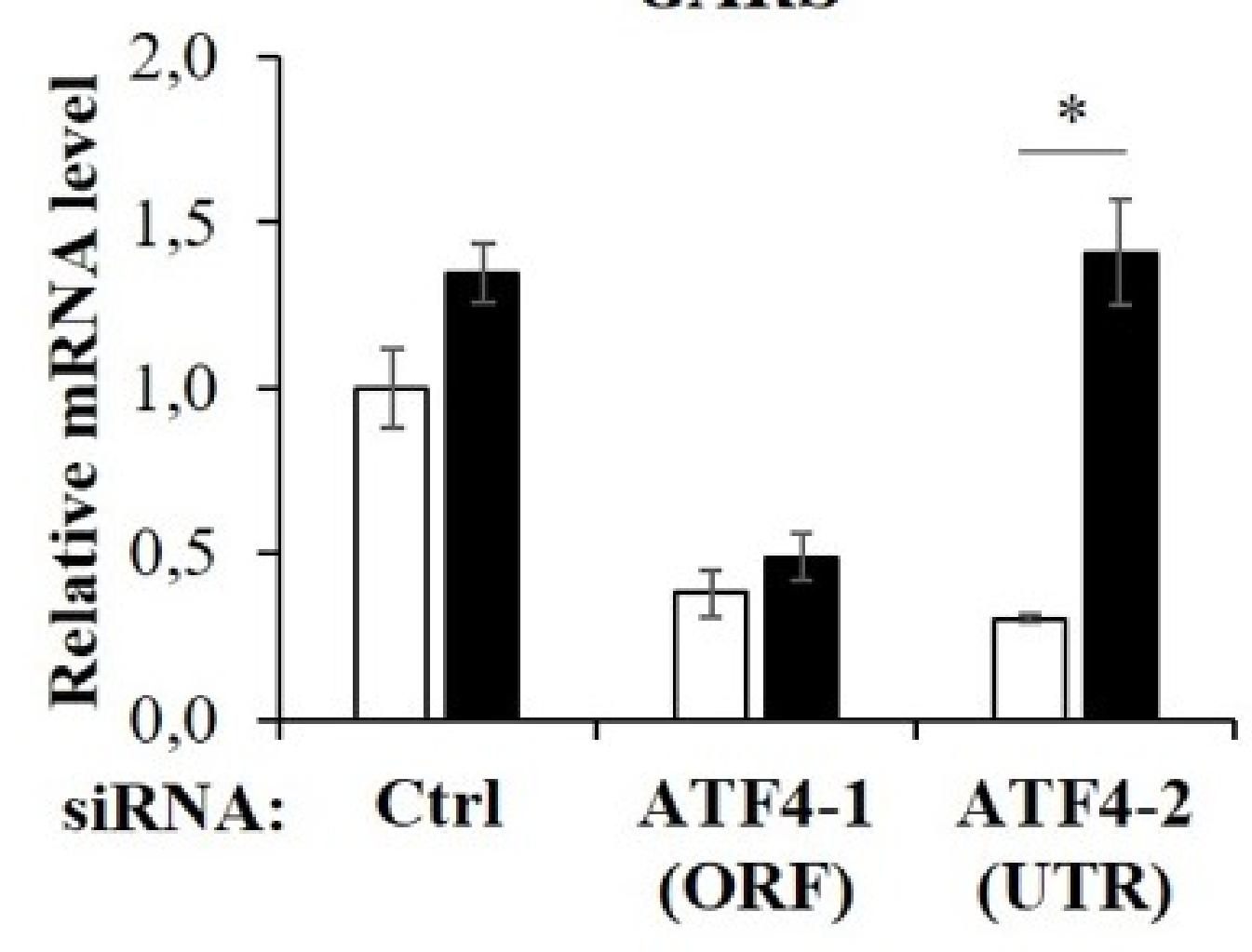

D

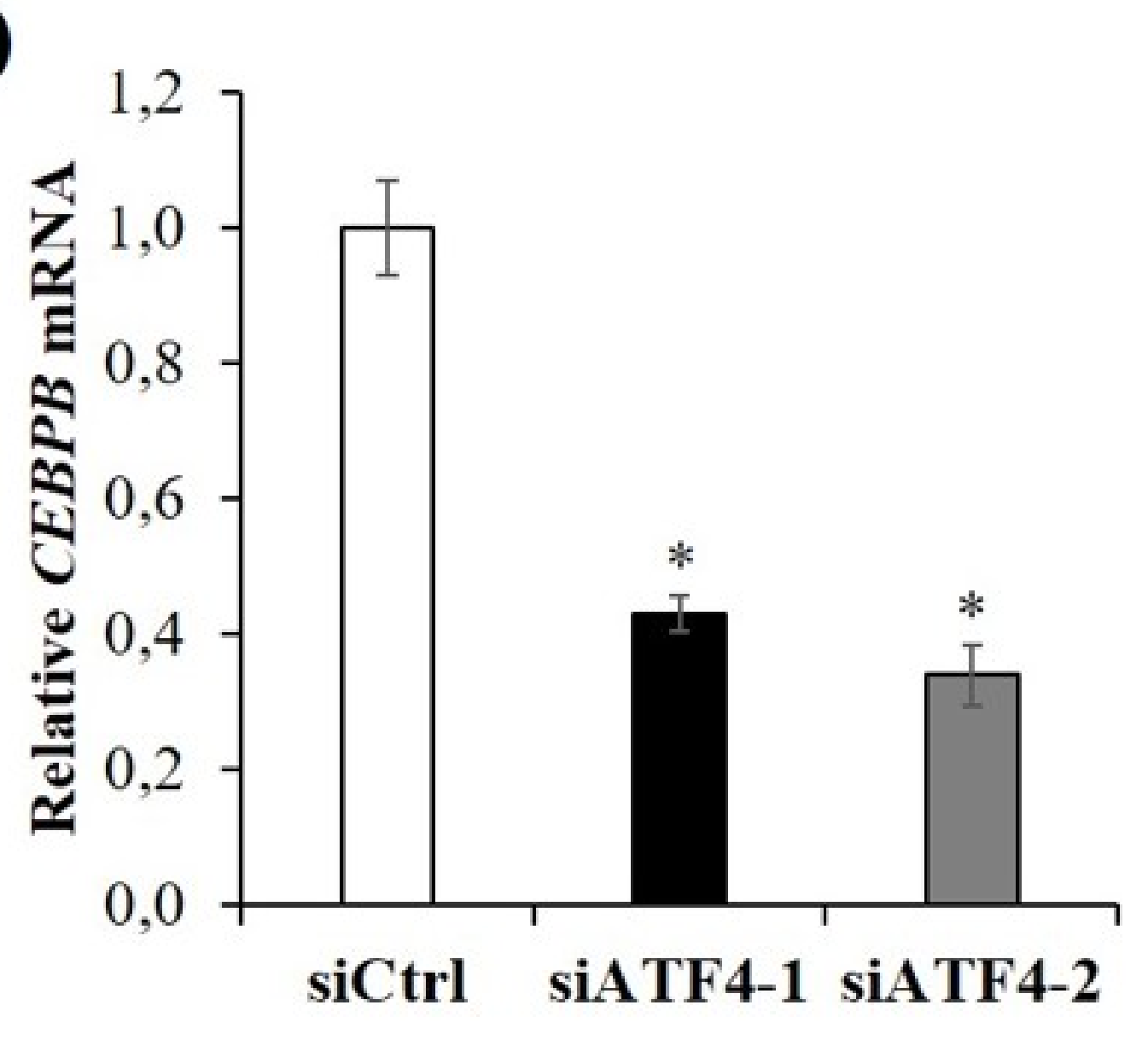

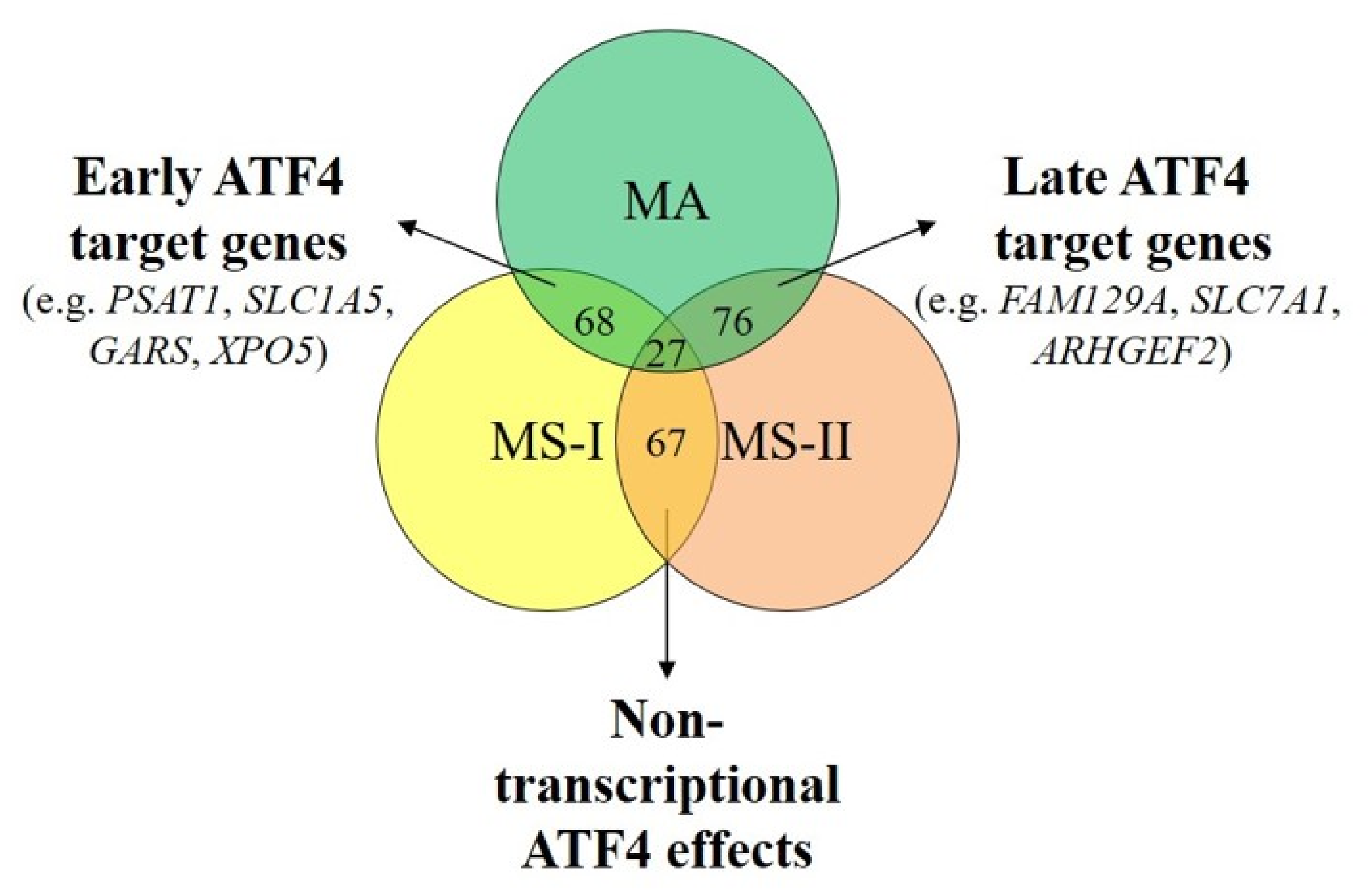

$\underline{\text { AA transporter }}$

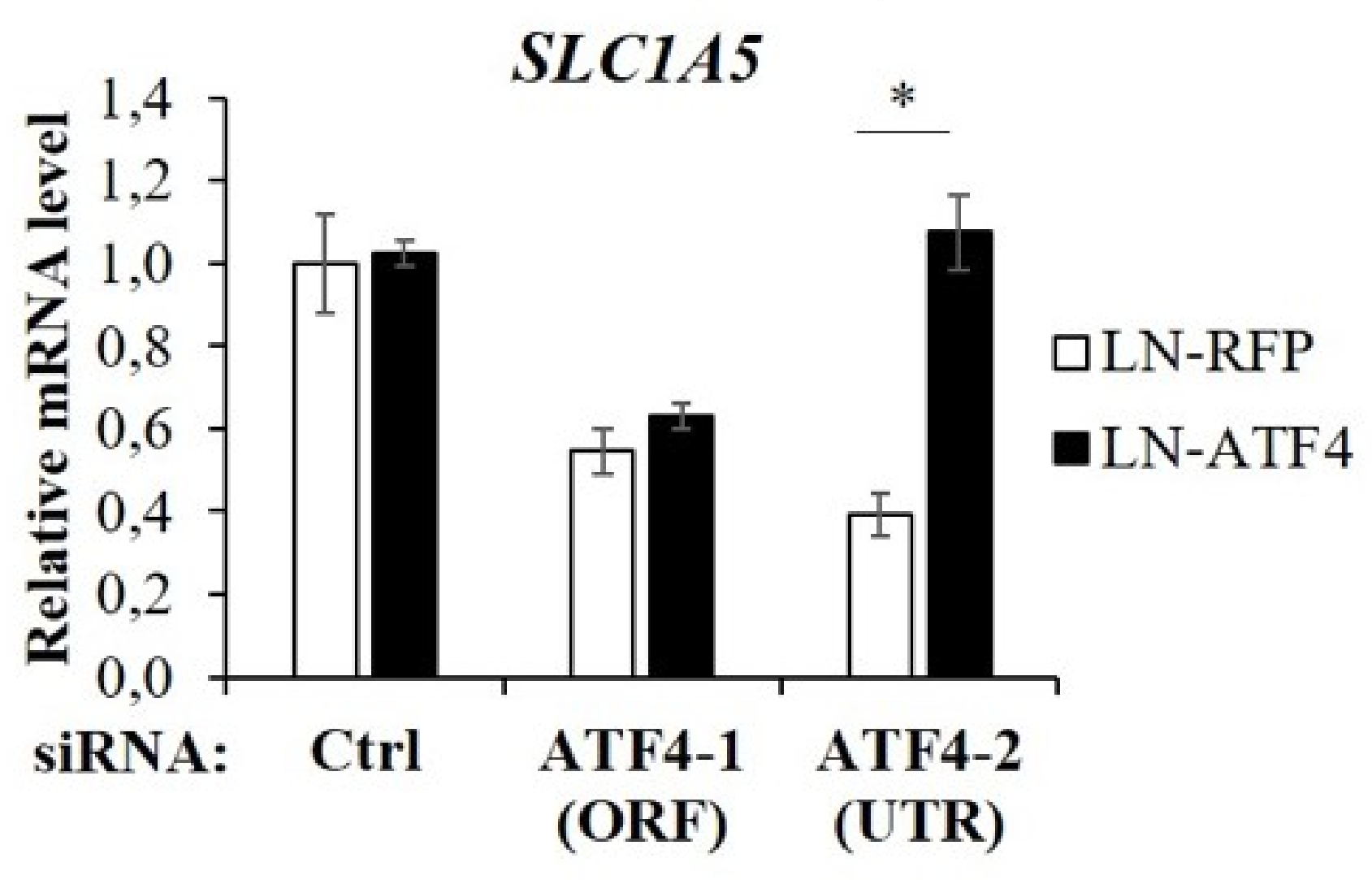

tRNA exporter

XPO5

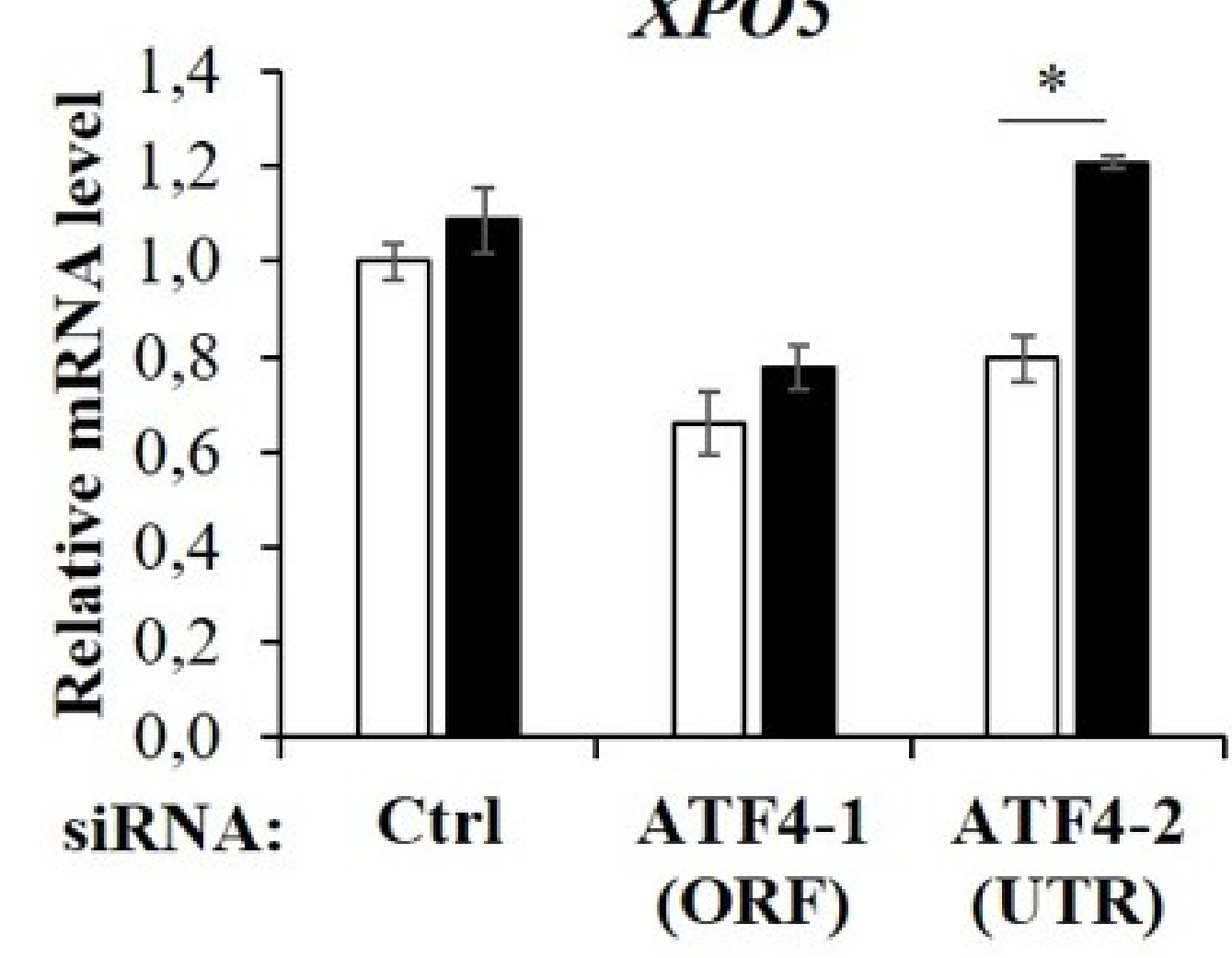

E

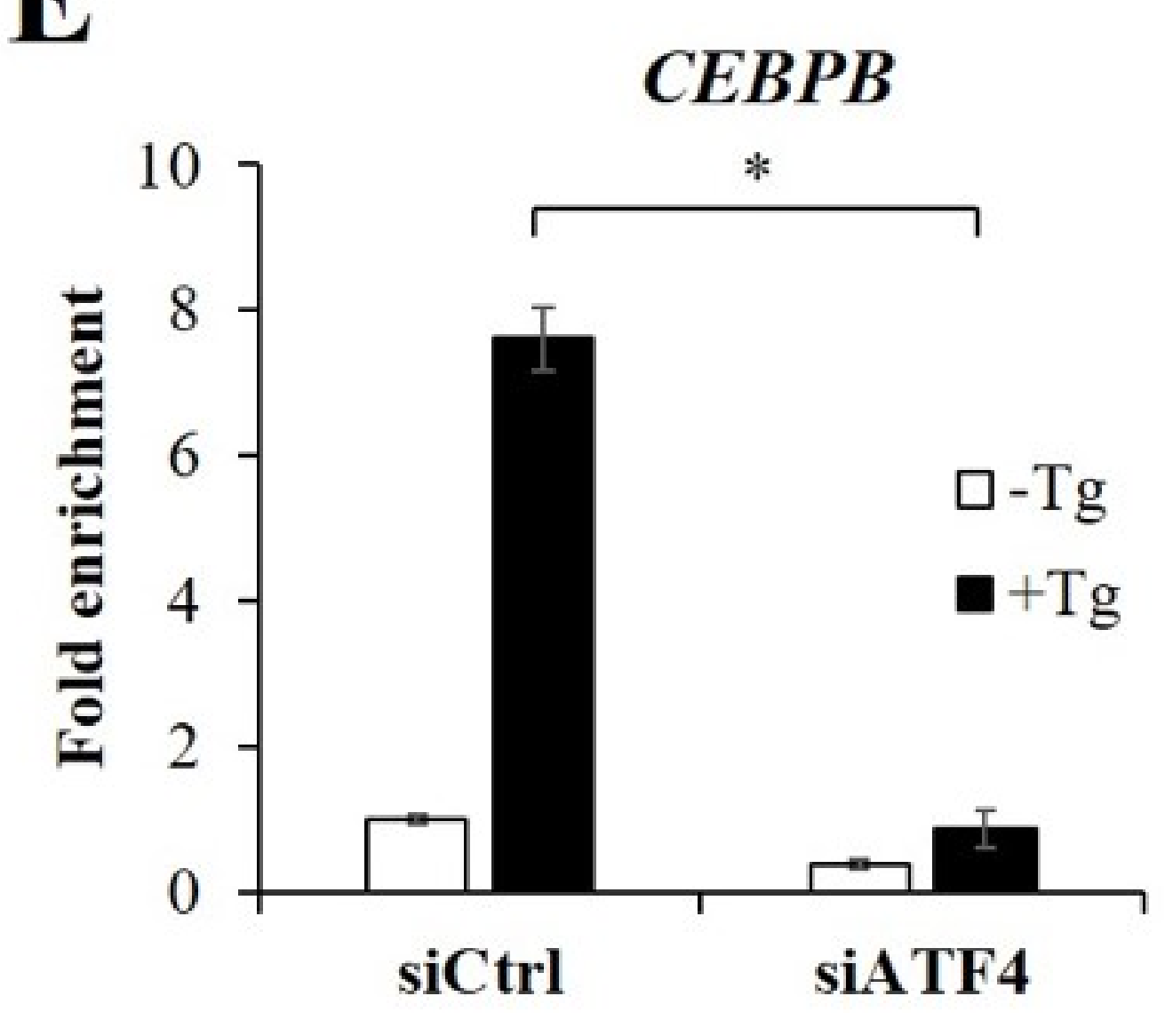


A

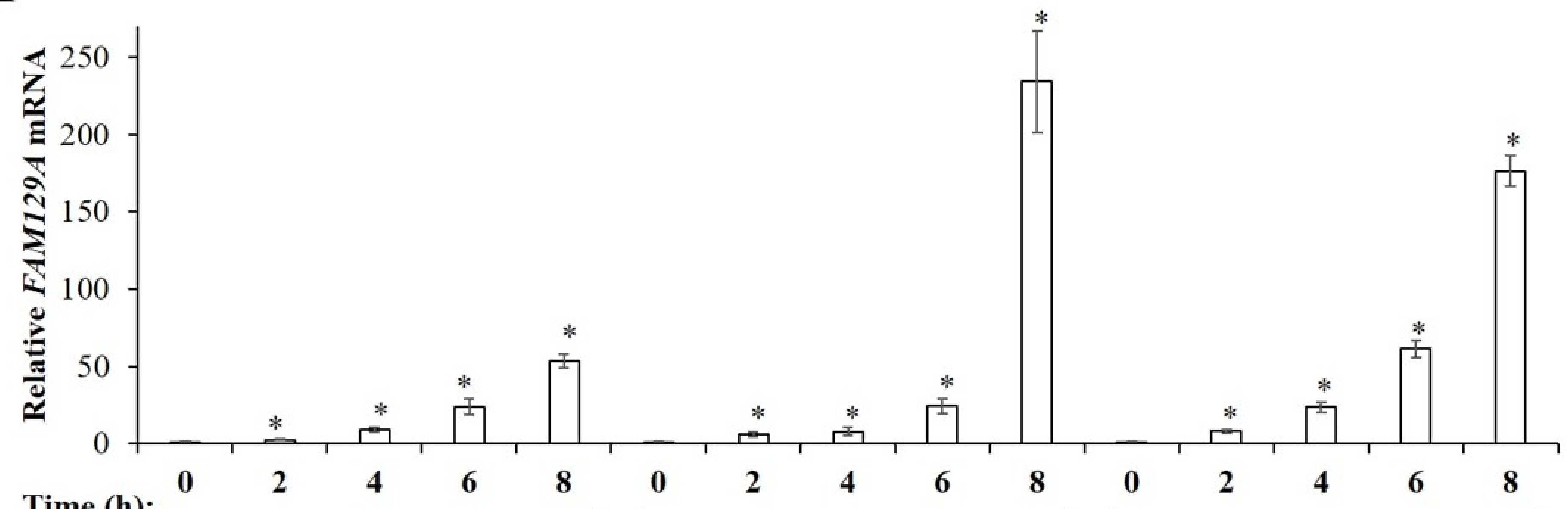

Time (h):

$100 \mathrm{nMTg}$

B

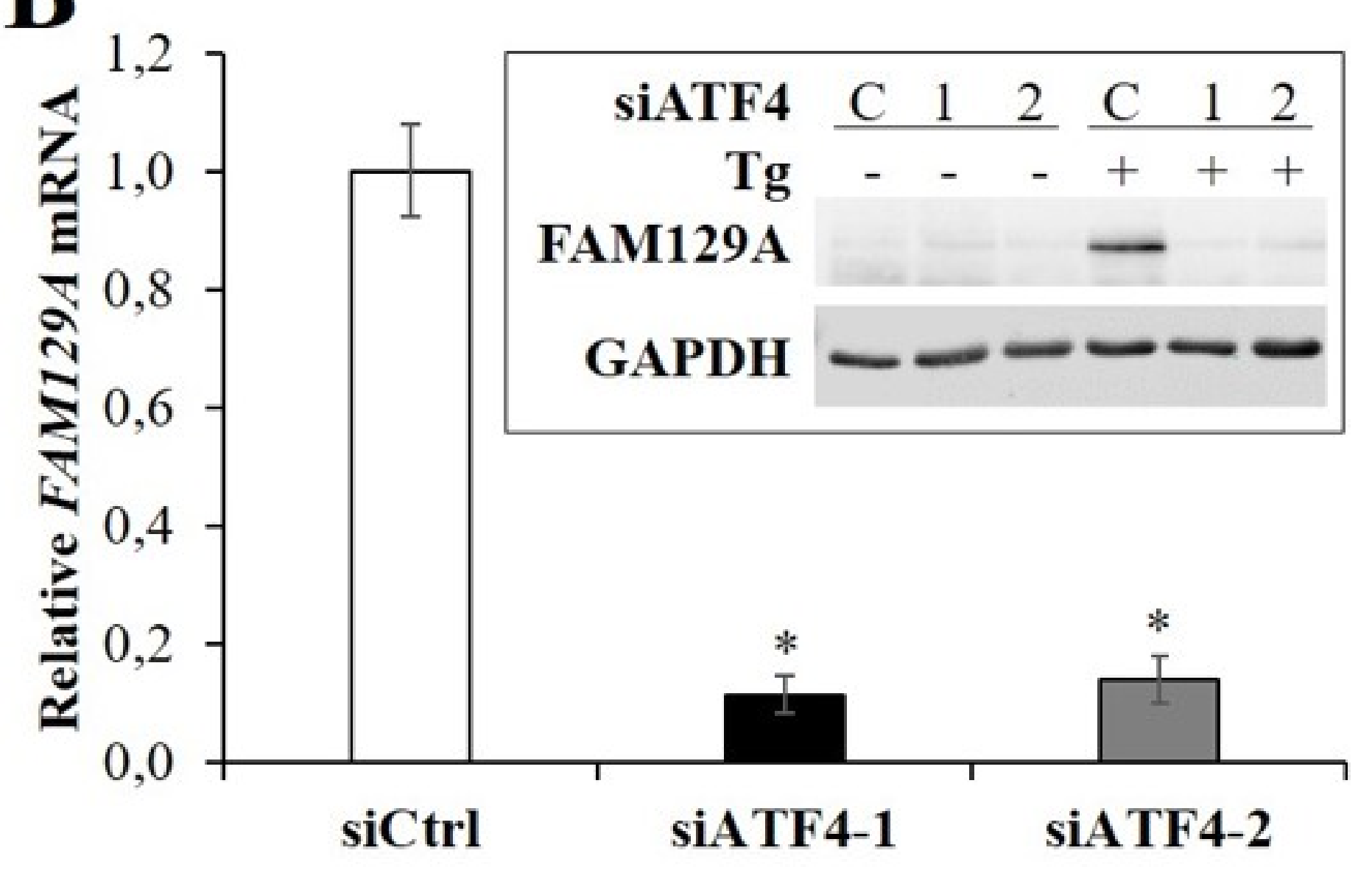

D

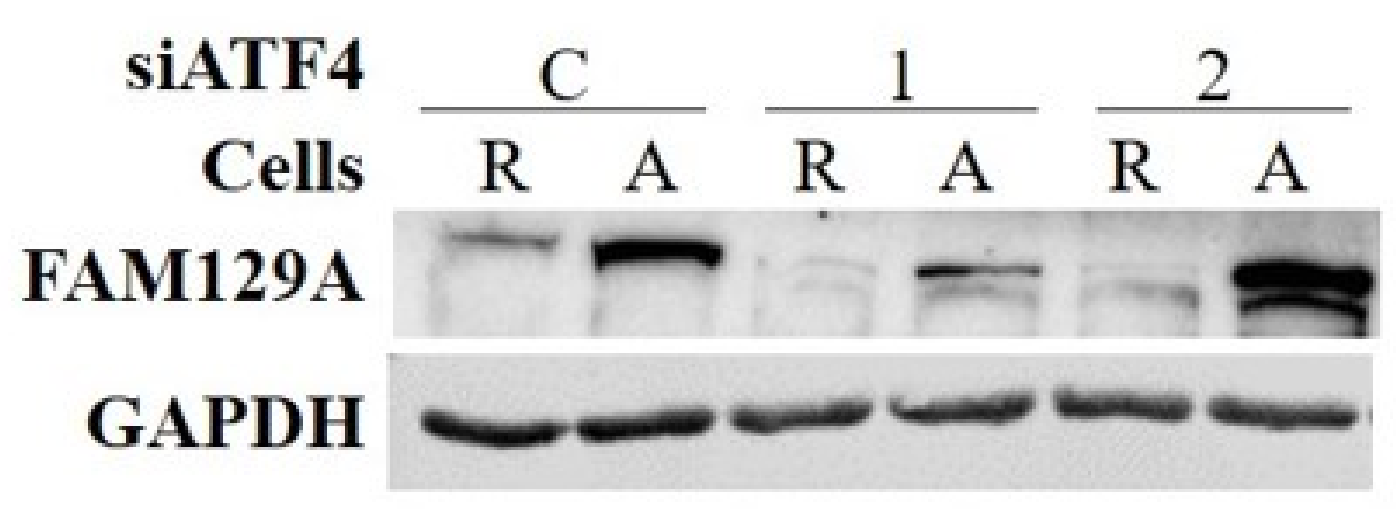

$5 \mu \mathrm{g} / \mathrm{mL}$ Tm

$50 \mu \mathrm{M} \mathrm{Bfa}$

C

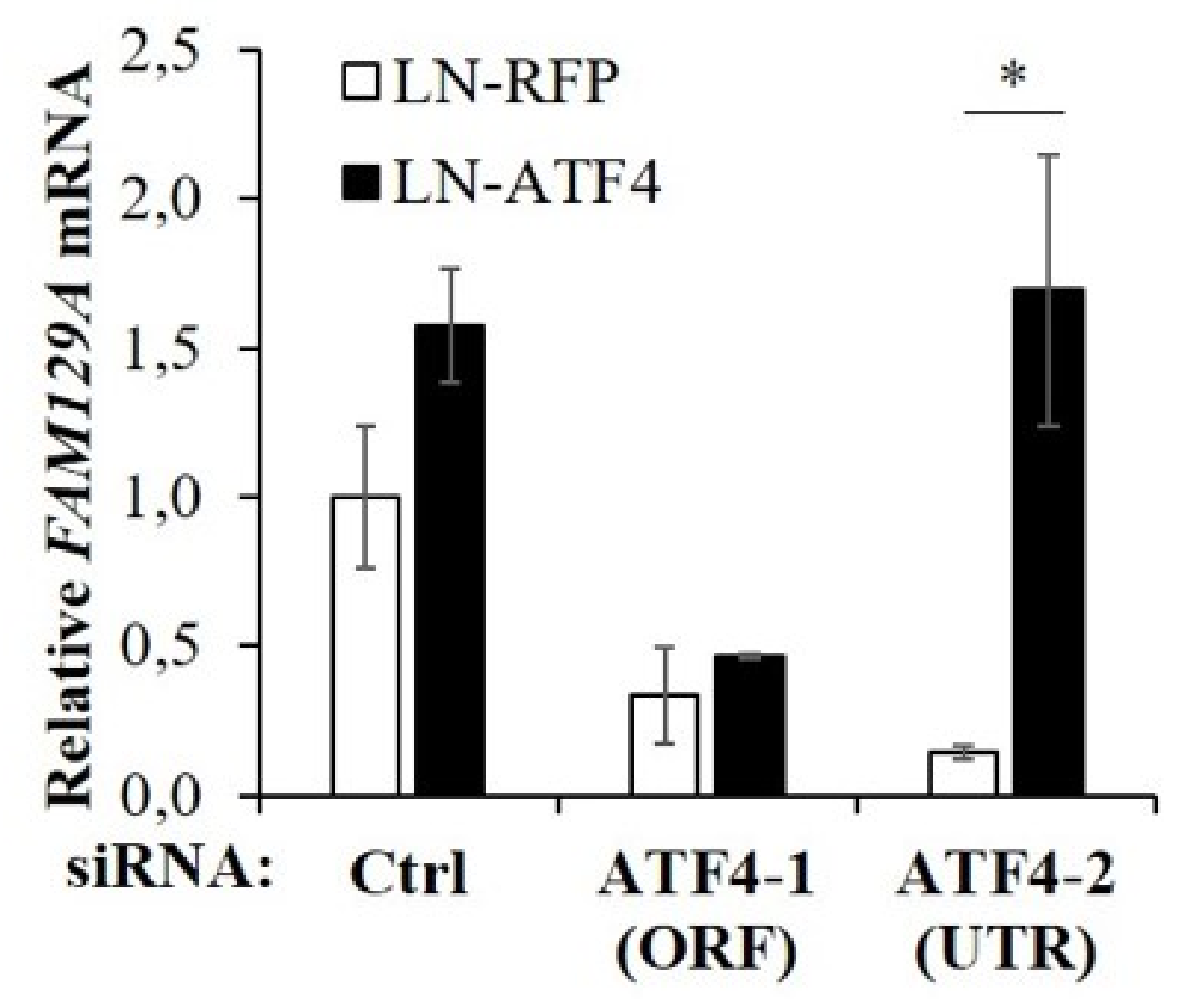

$\mathbf{E}$ FAM129A

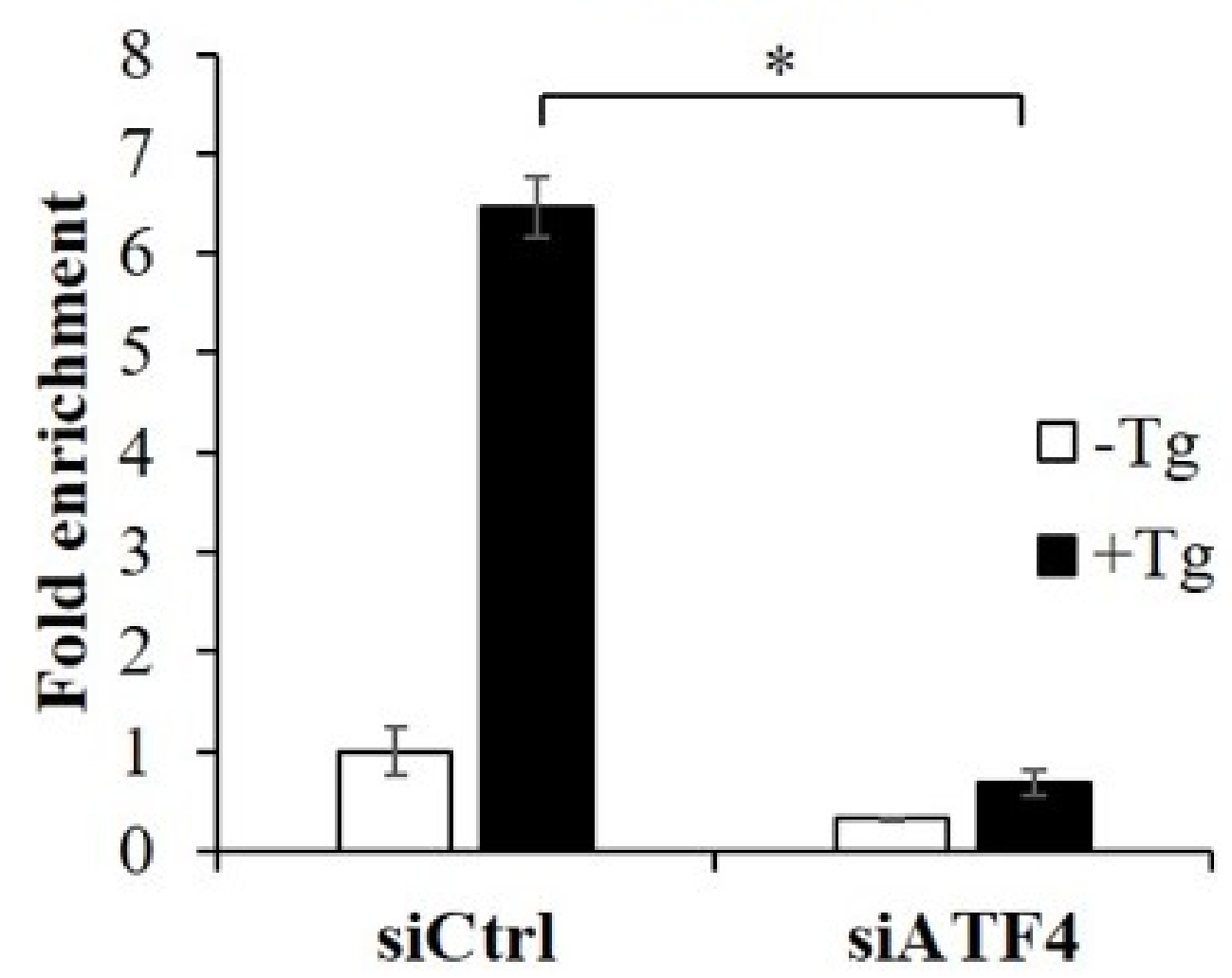

F

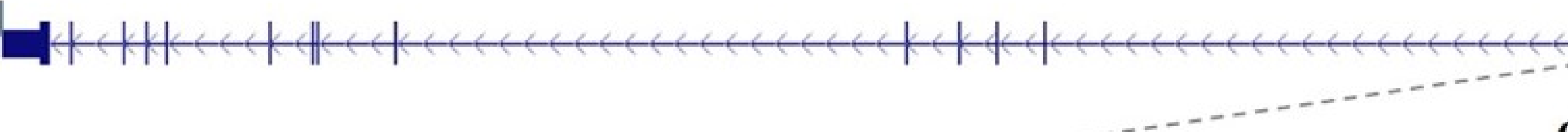


A
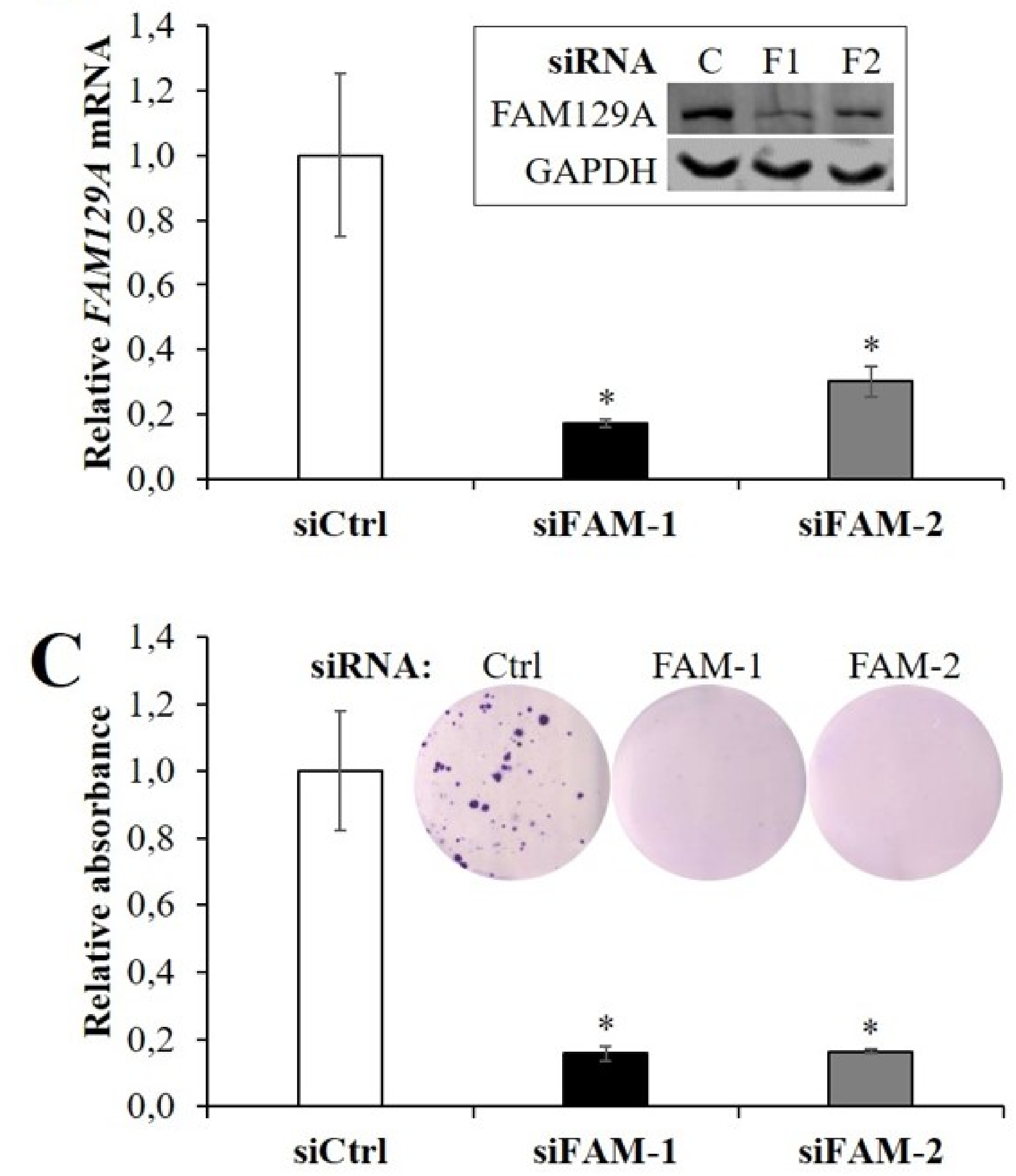

B

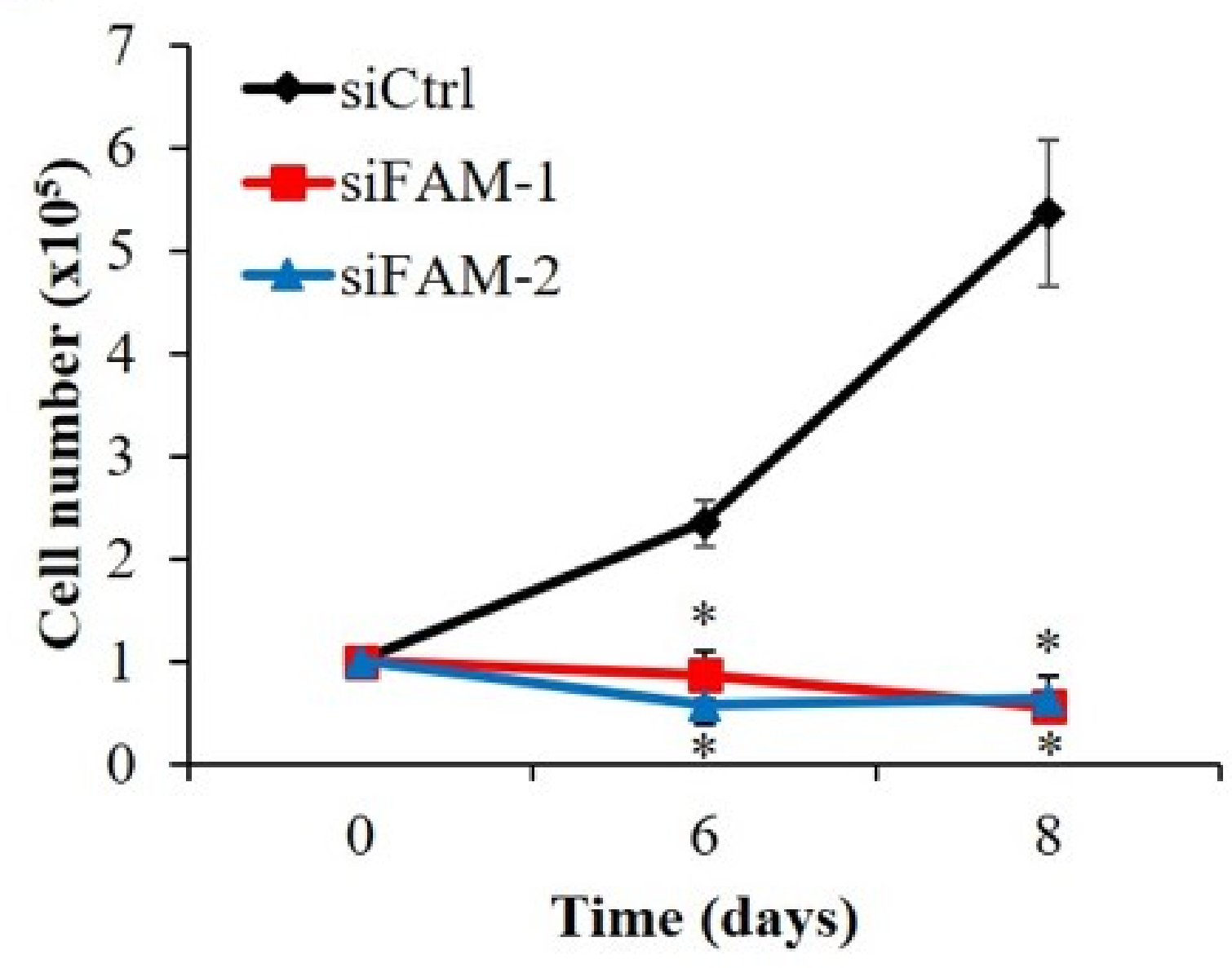

D

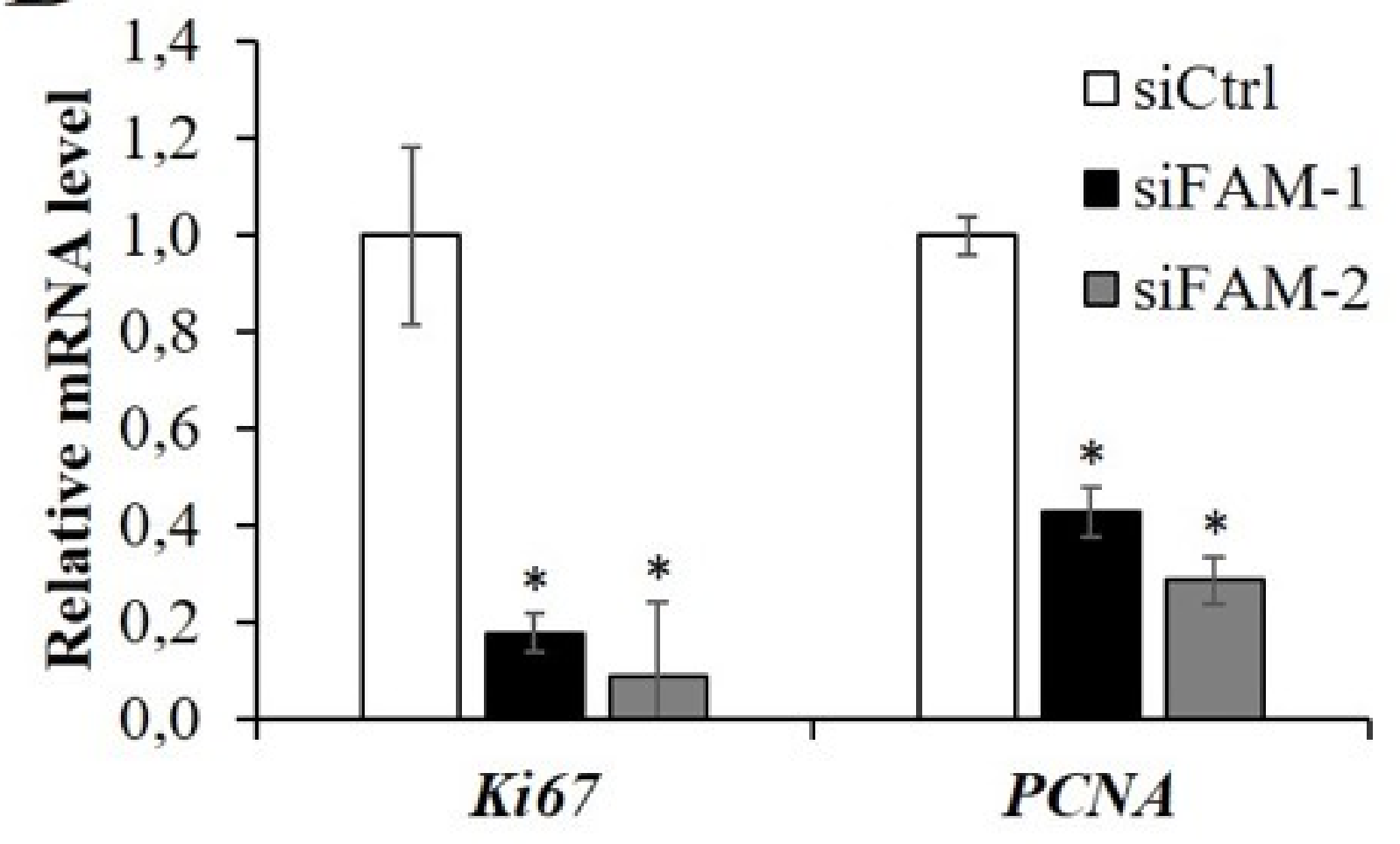

E

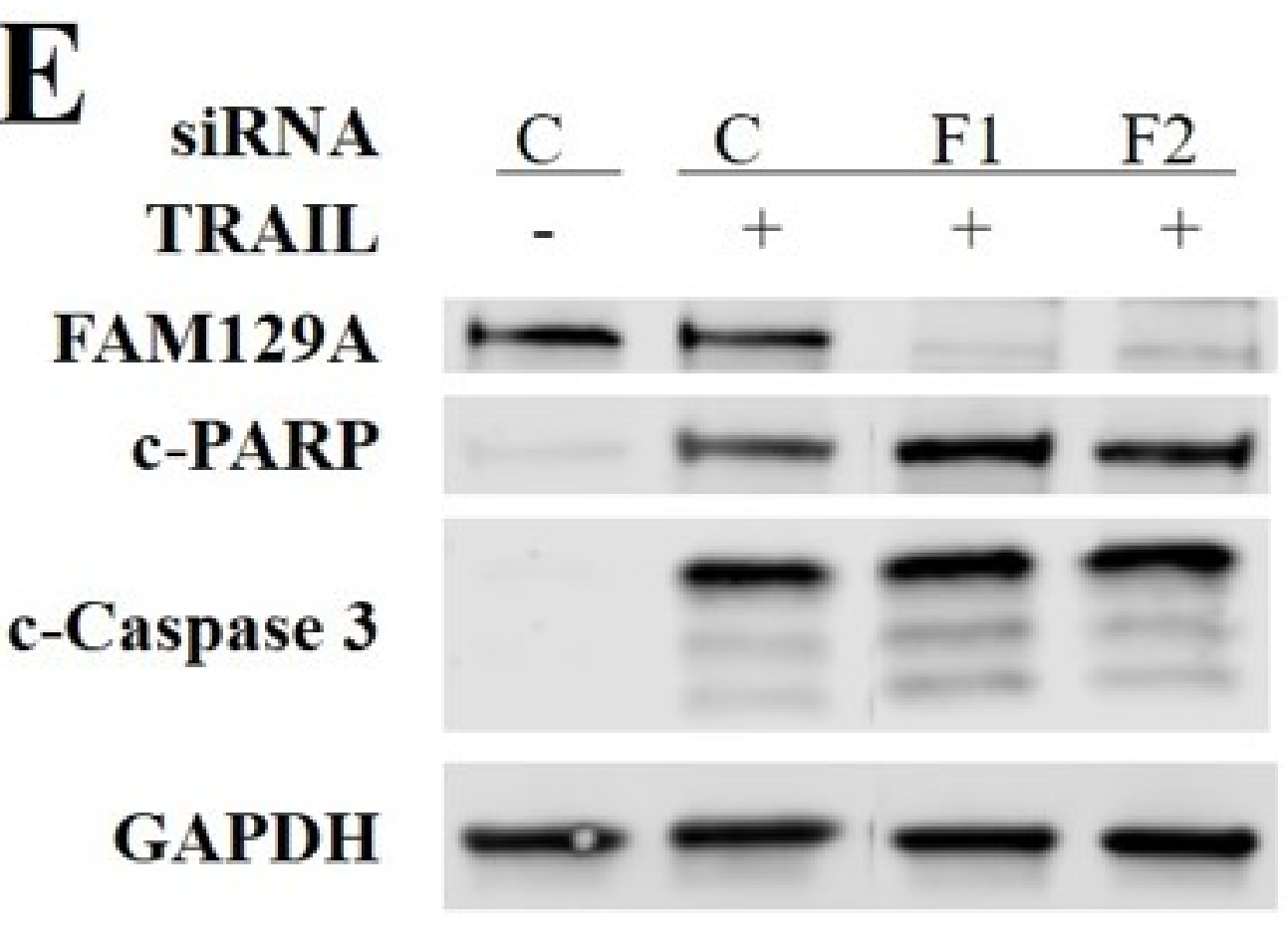

F

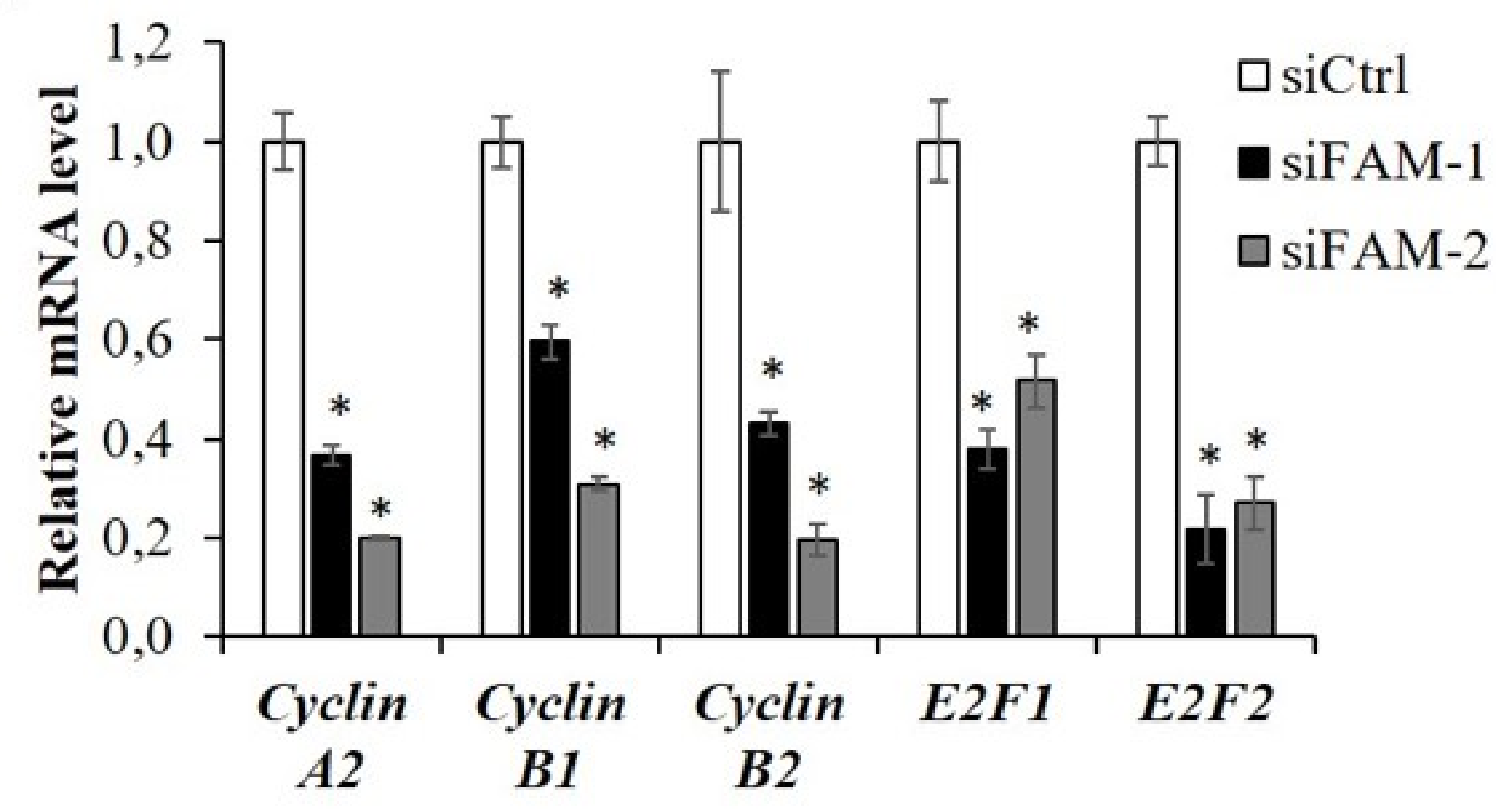

G

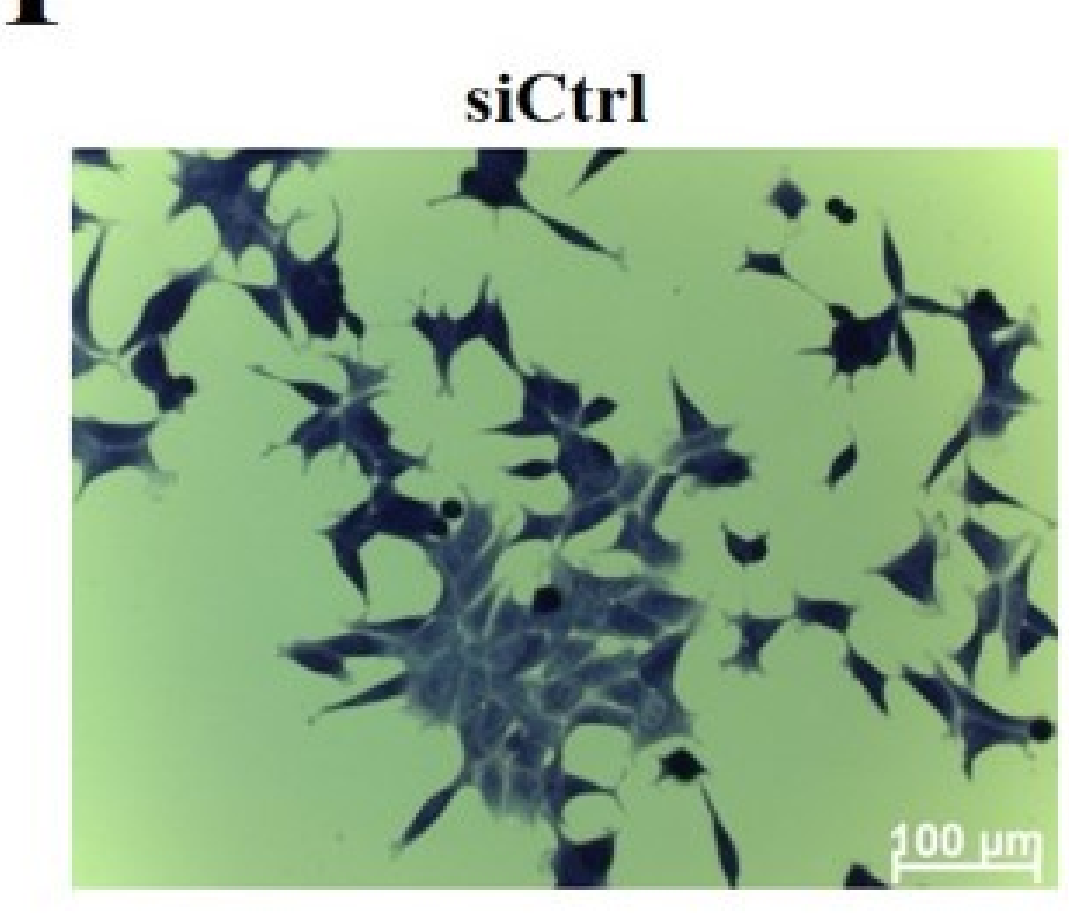

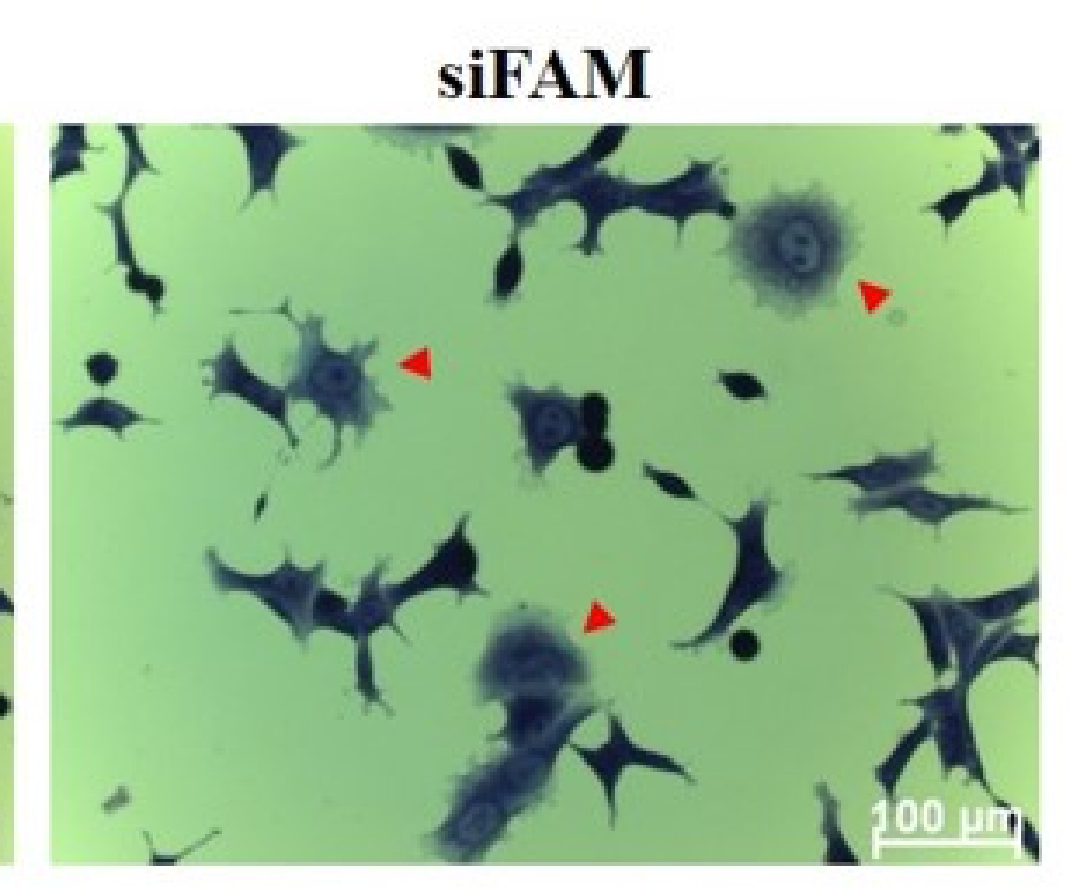

H

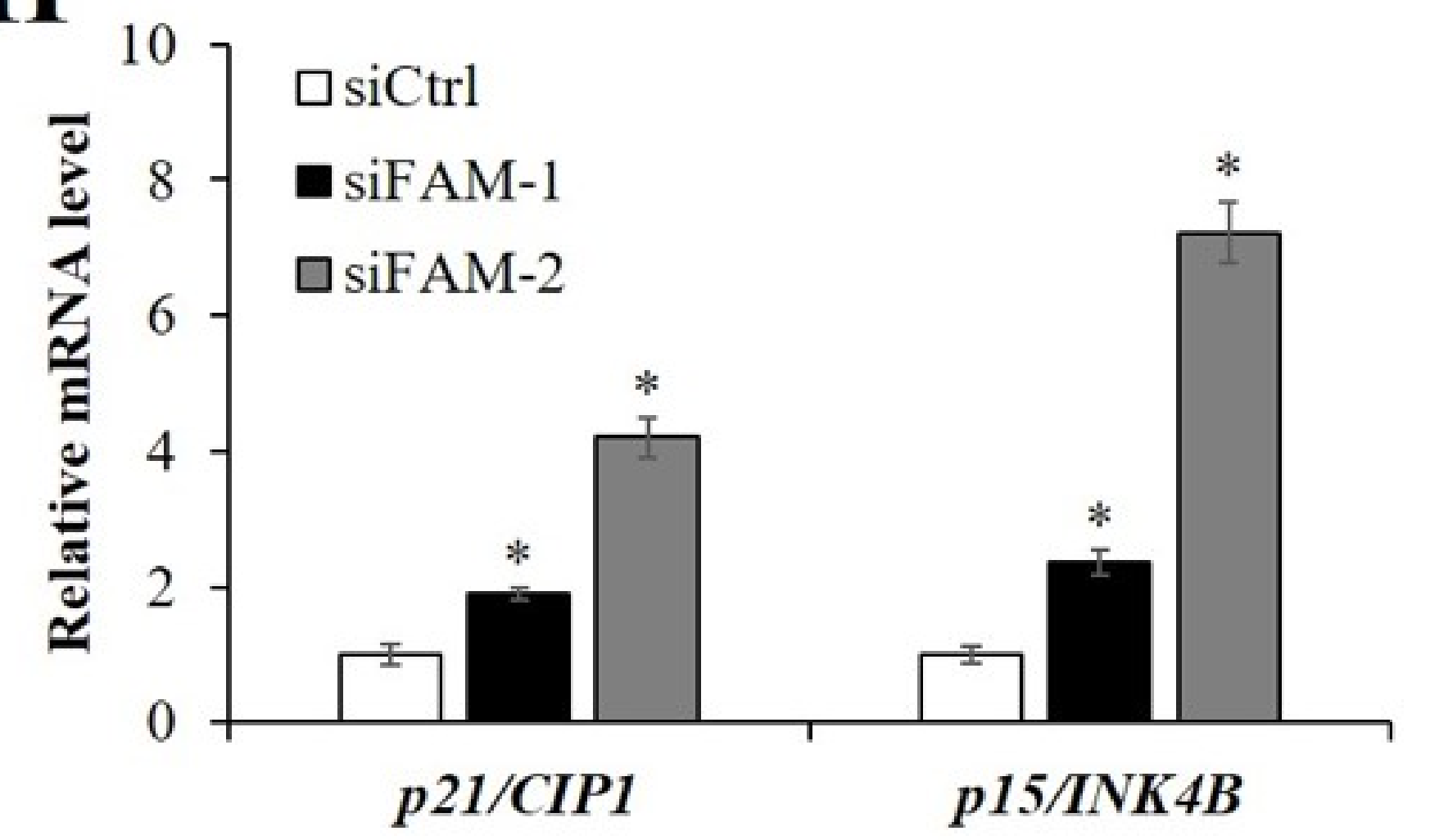

J

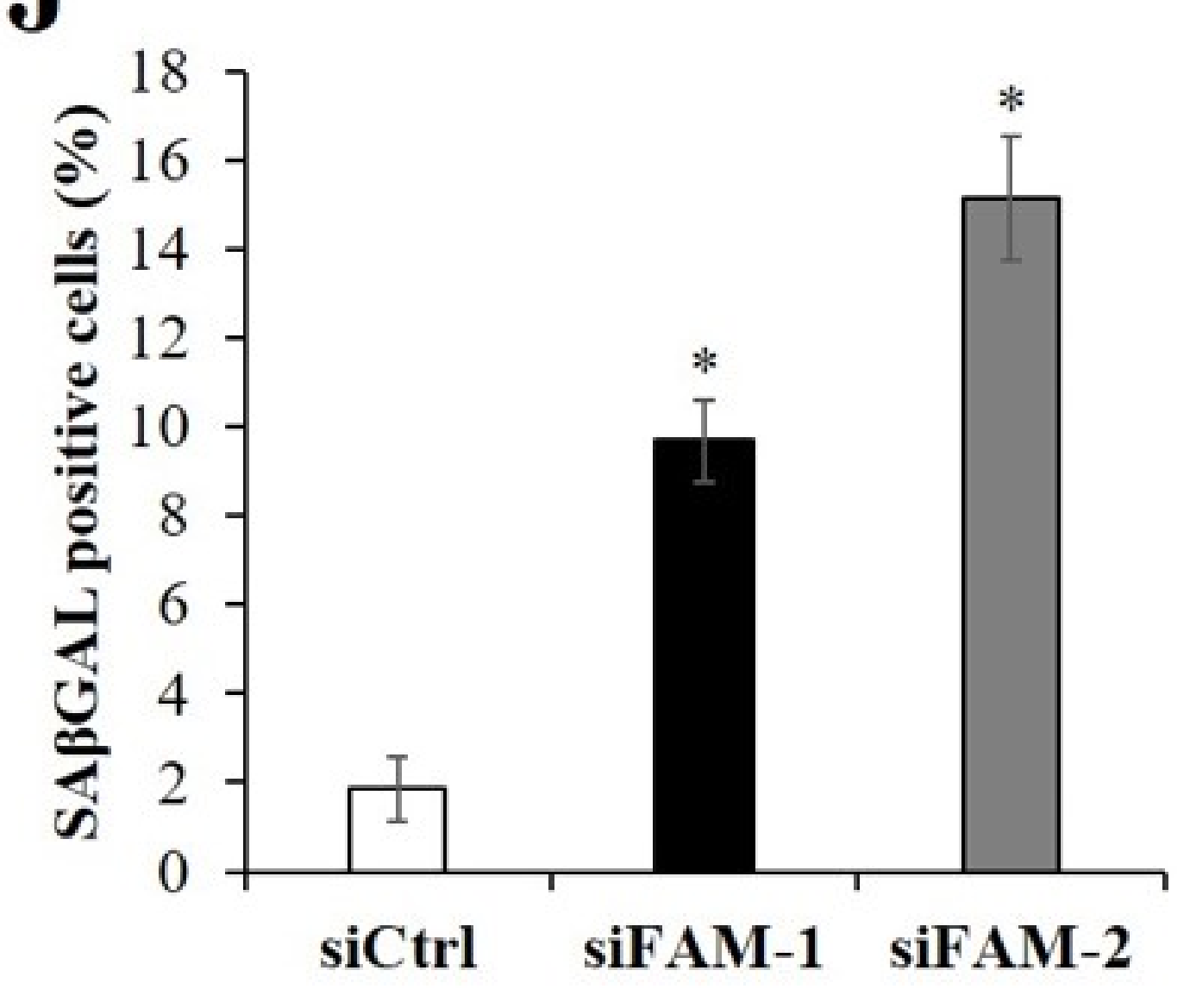




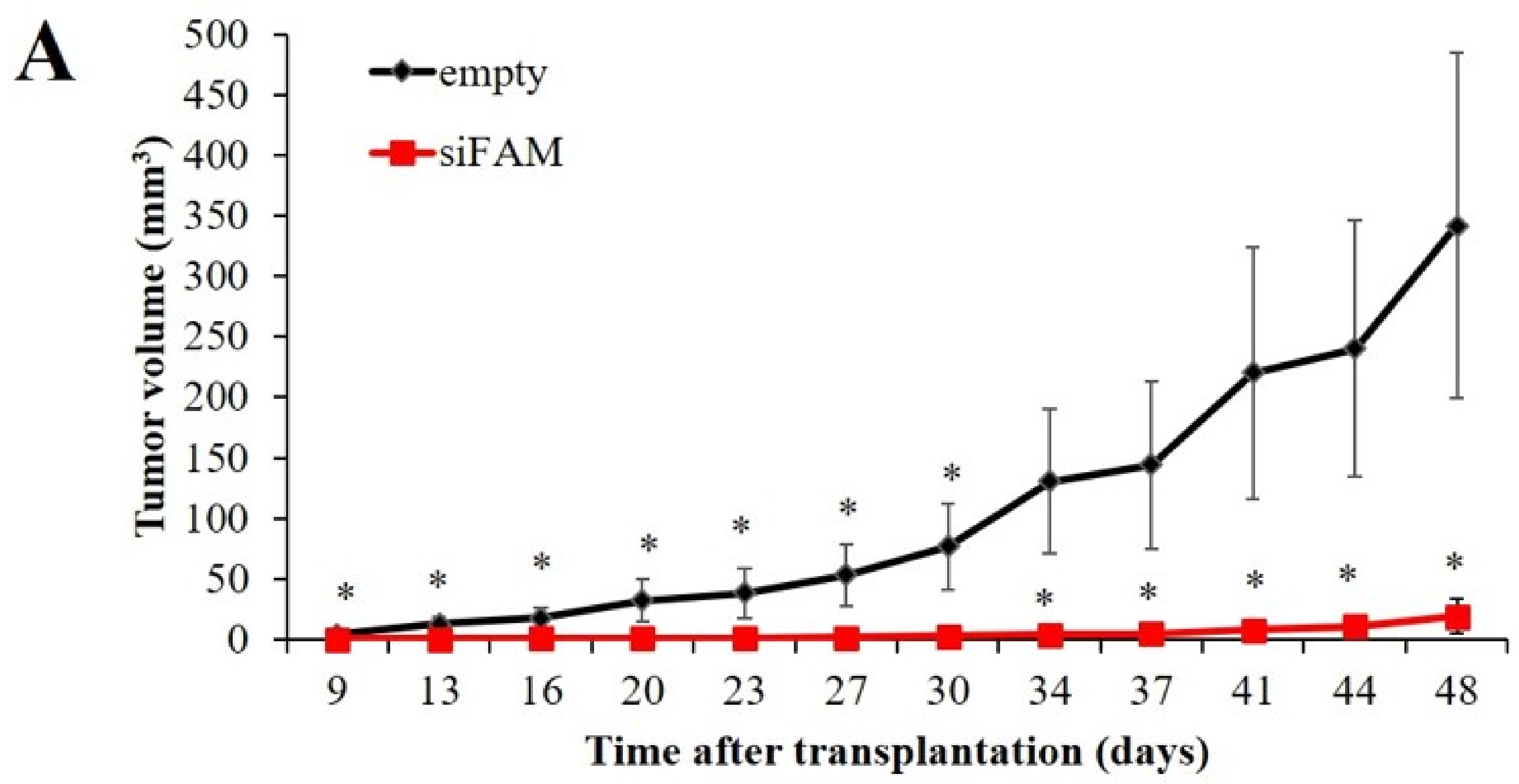

B

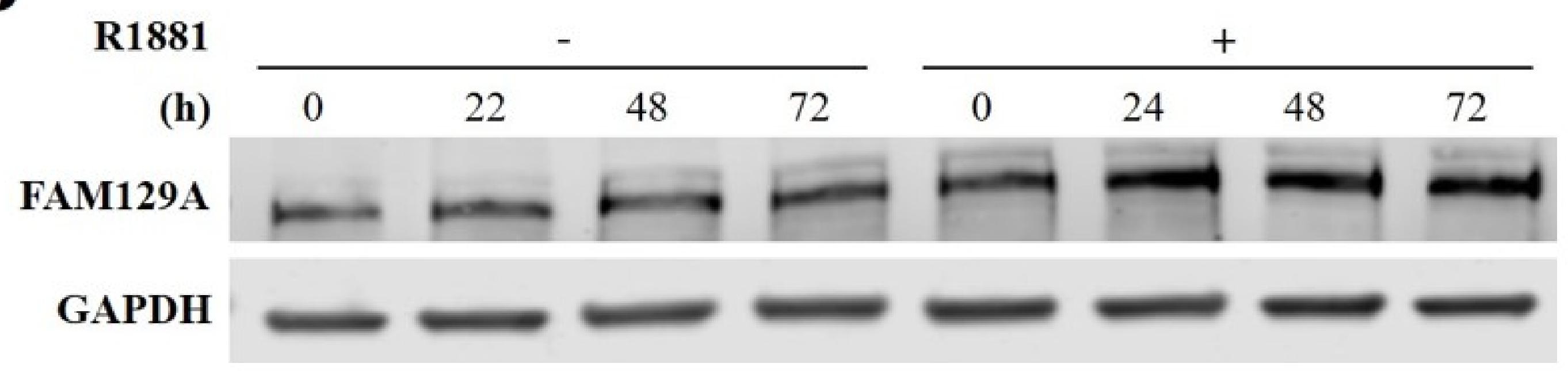

C
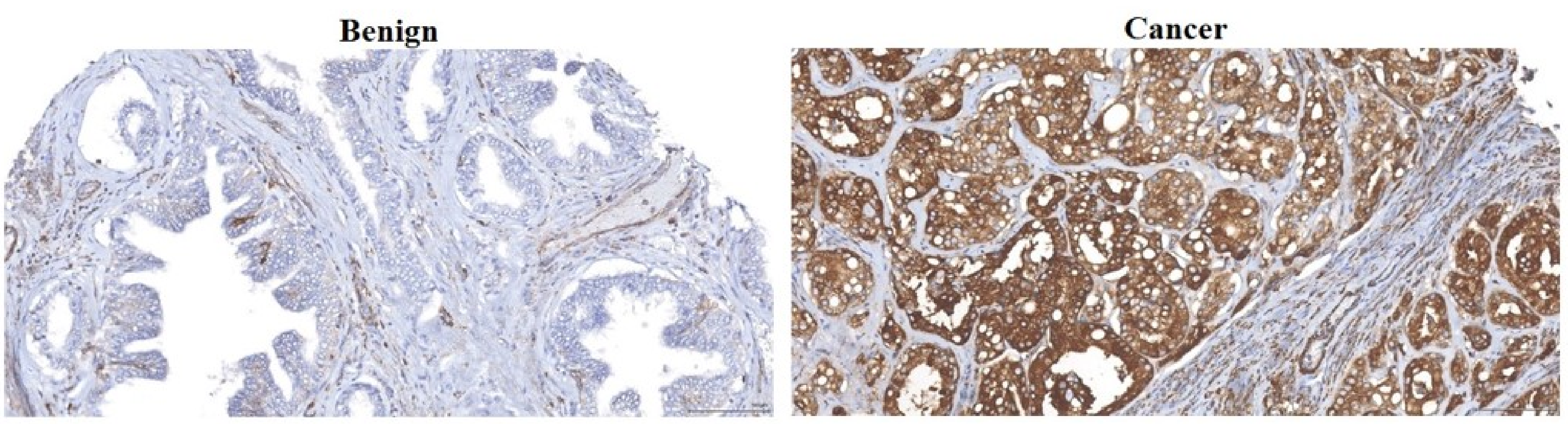

D

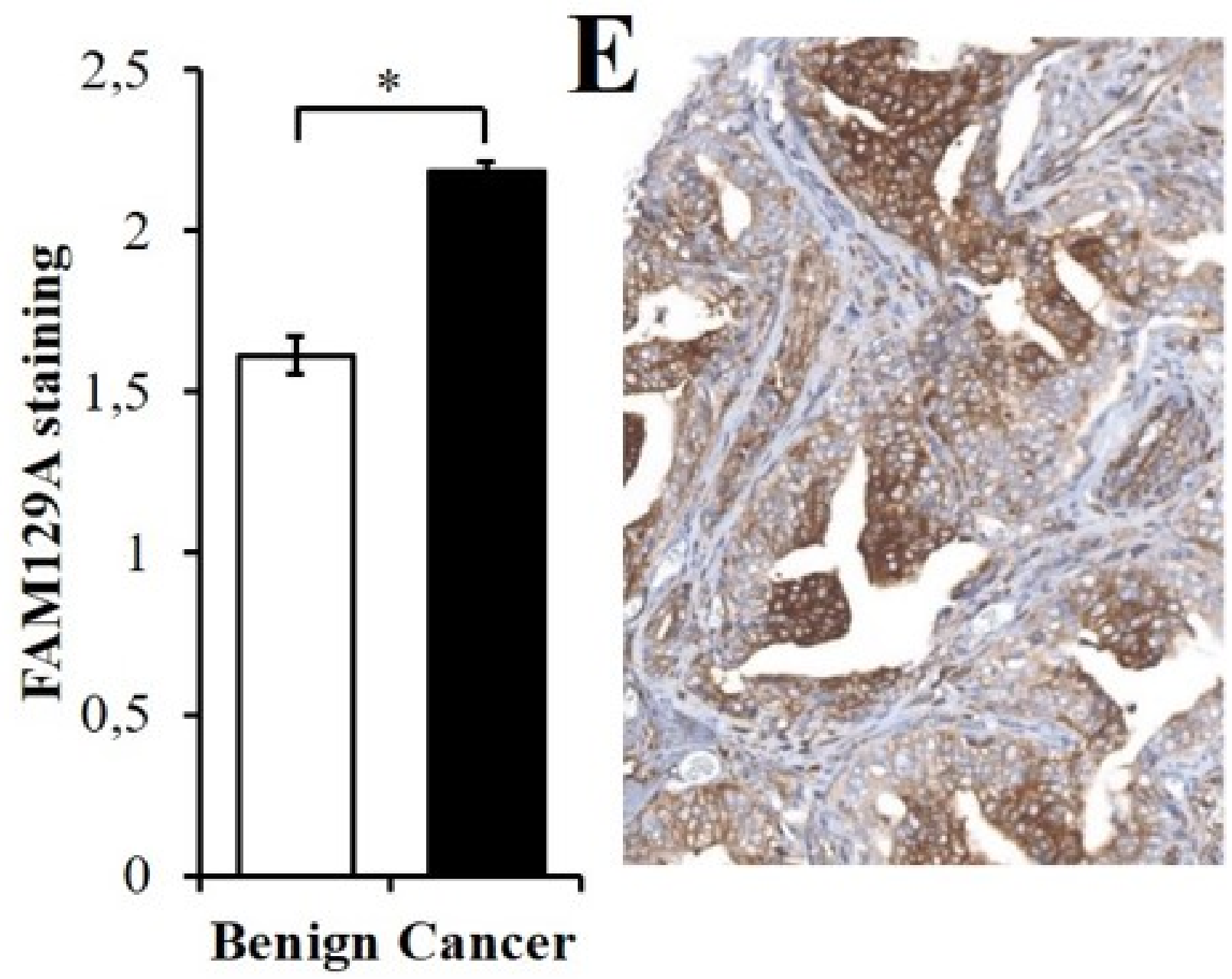

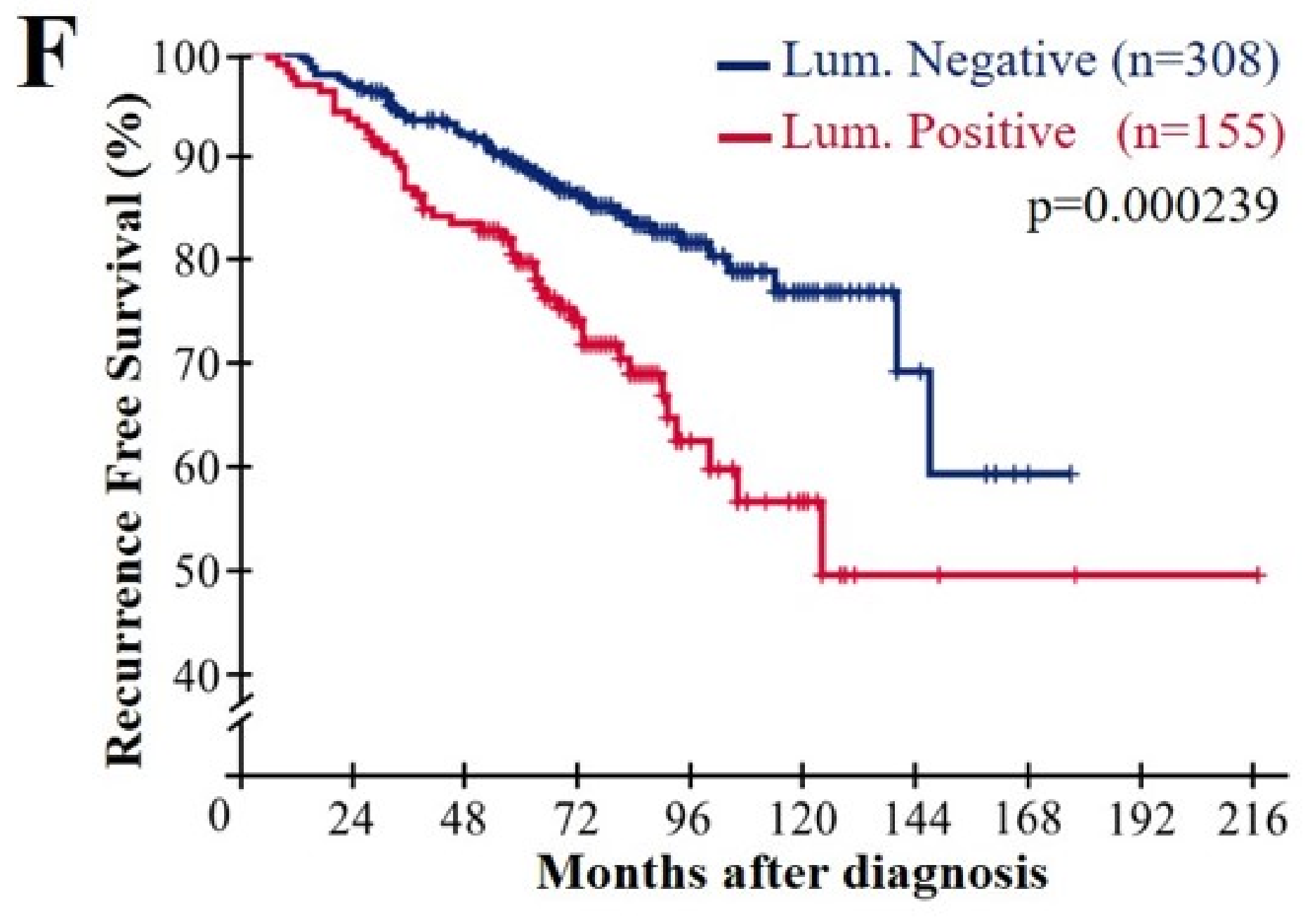



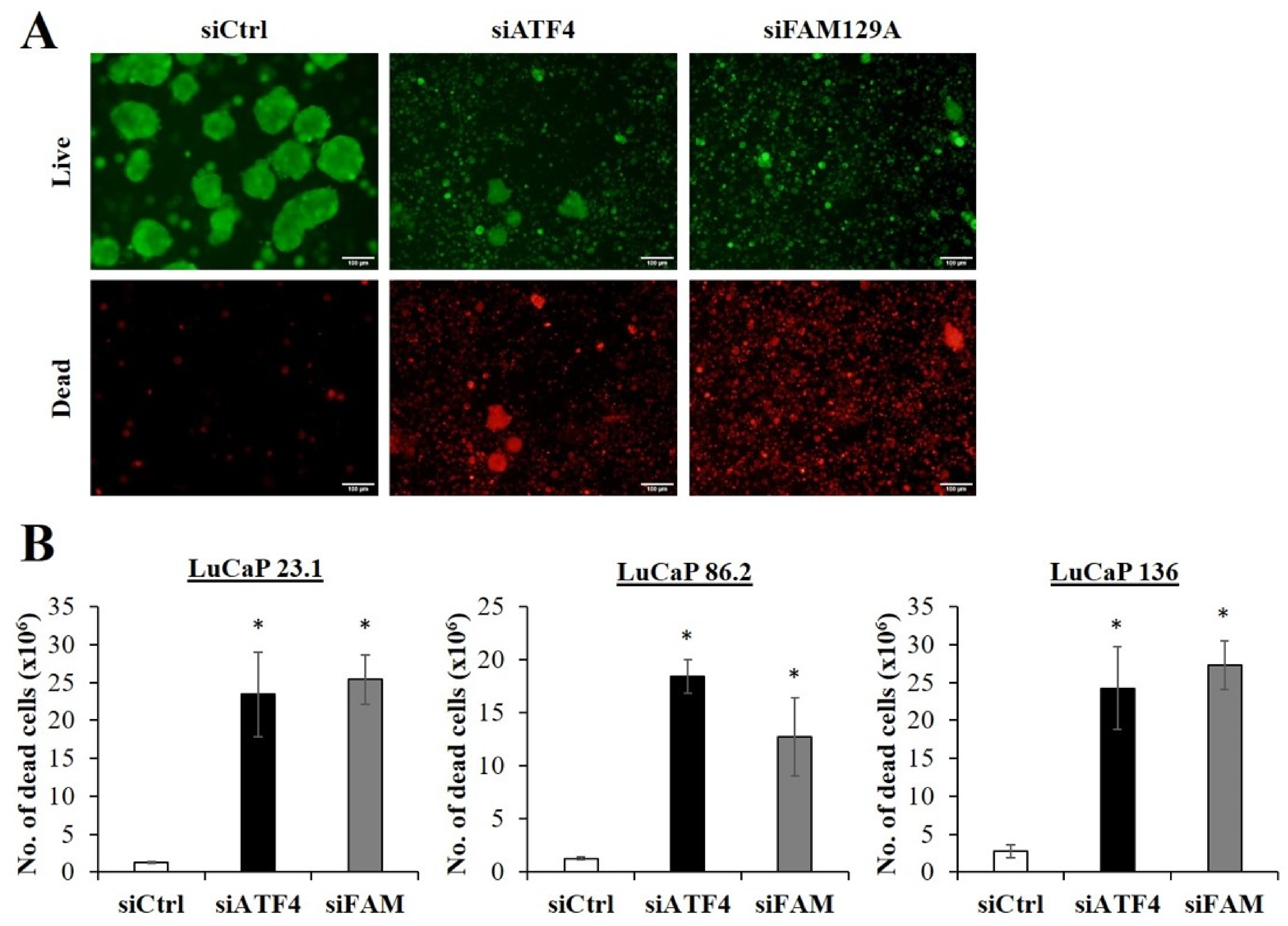
\begin{tabular}{lllllll} 
SiRNA & $\mathrm{C}$ & $\mathrm{F} 1$ & $\mathrm{~F} 2$ & $\mathrm{C}$ & $\mathrm{F} 1$ & $\mathrm{~F} 2$ \\
\hline
\end{tabular}

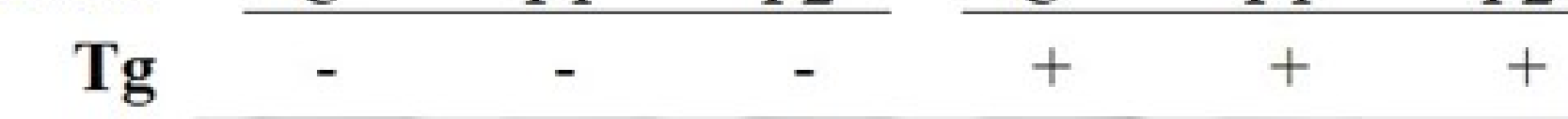

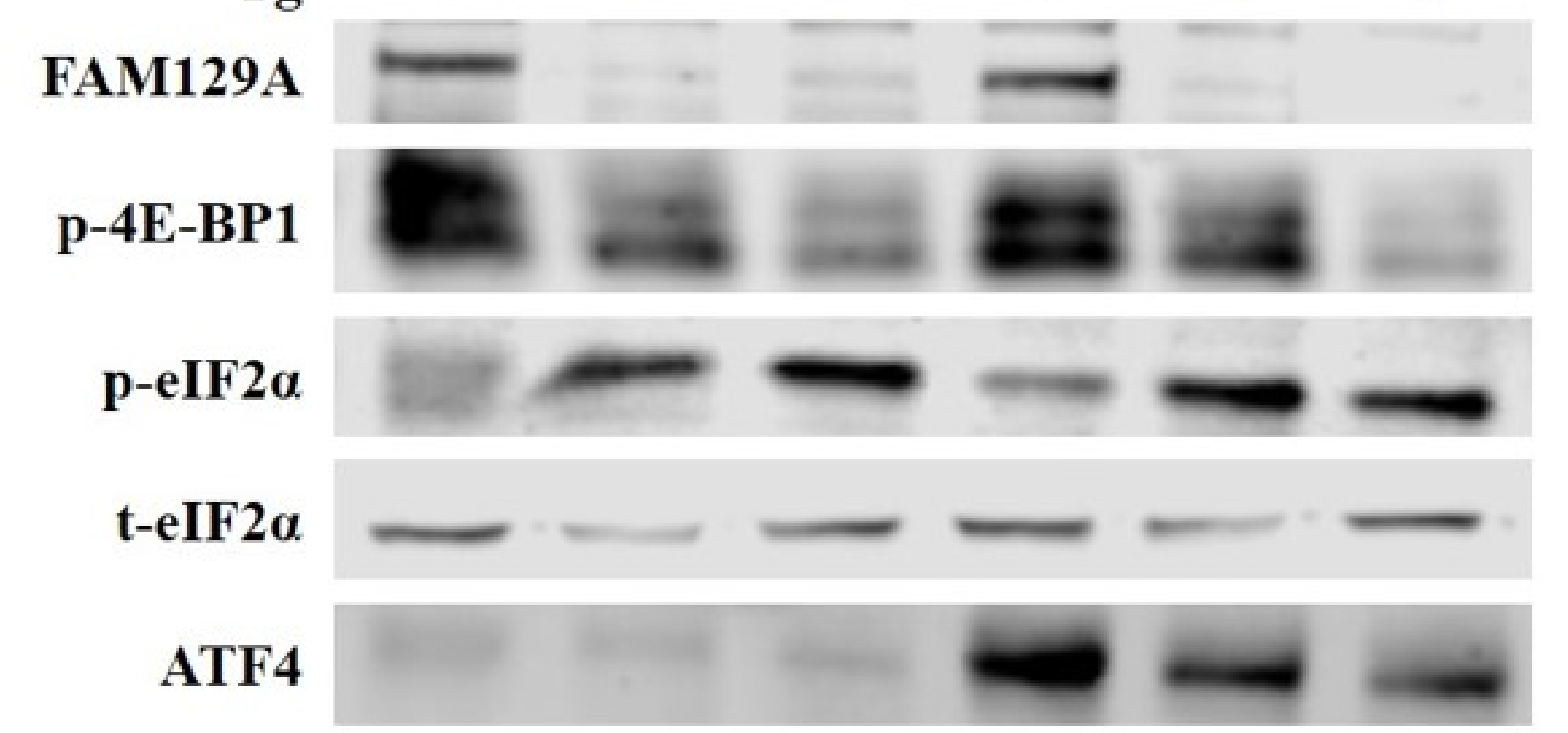

CHOP

GAPDH

C

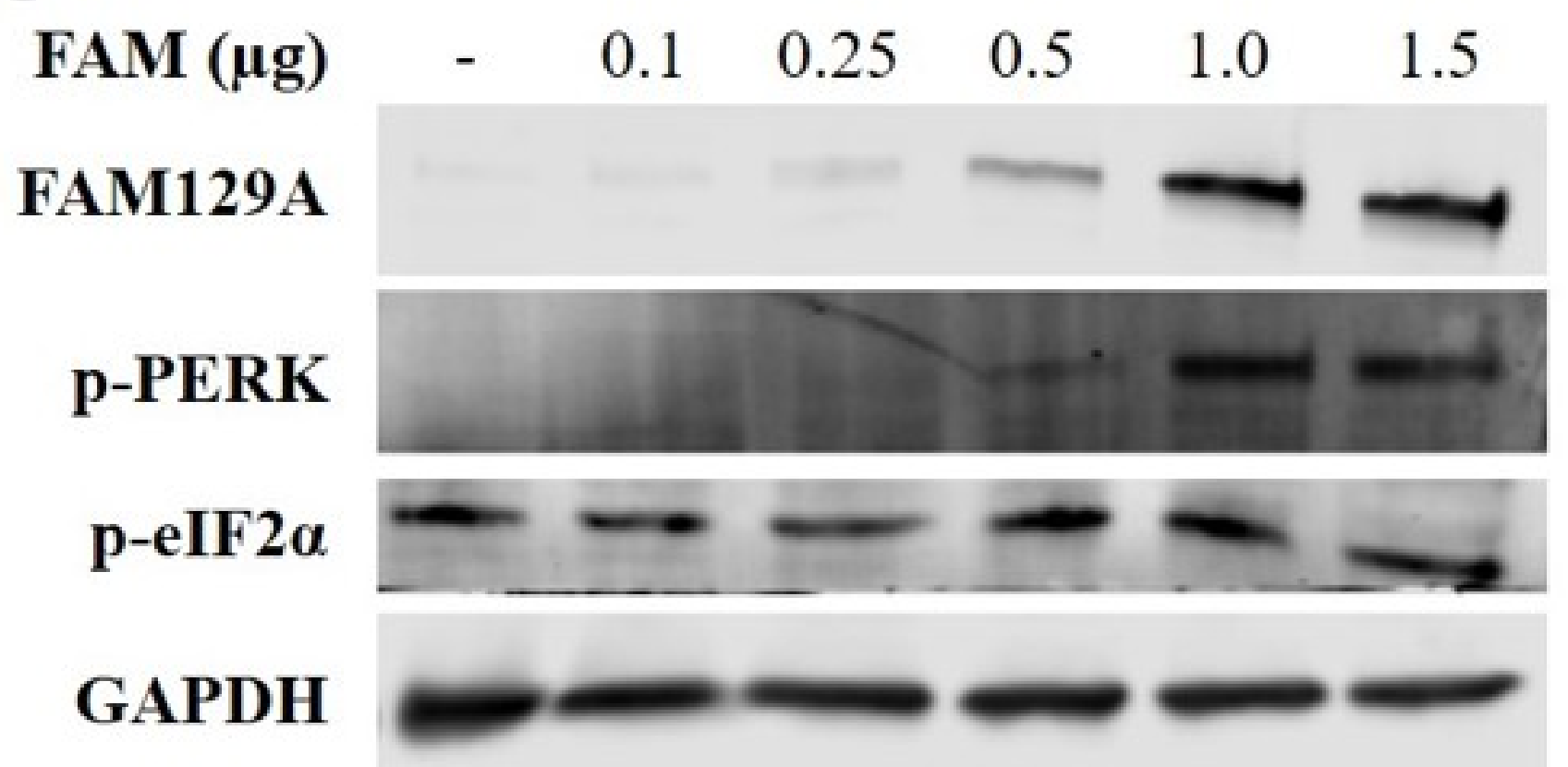

D

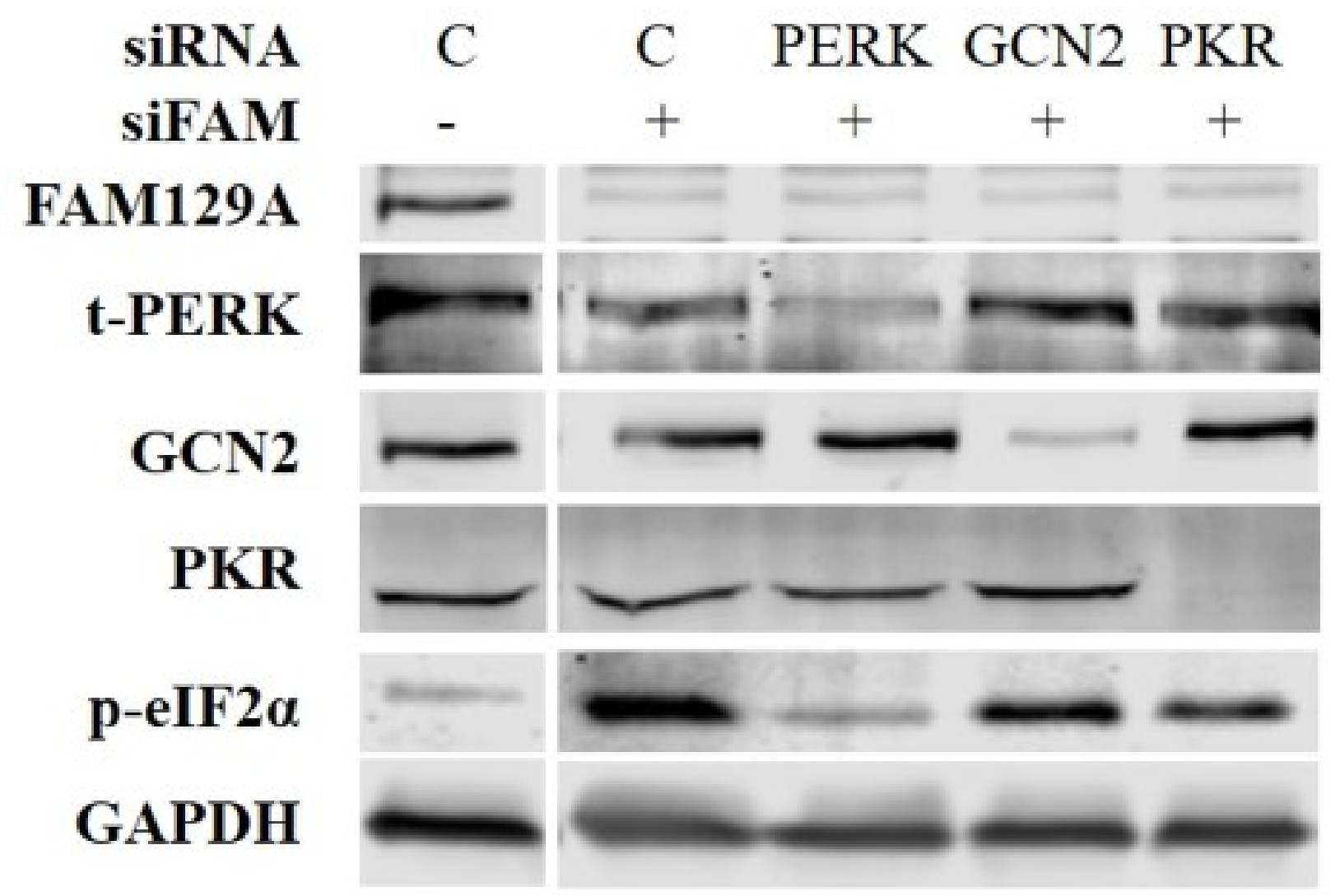

E

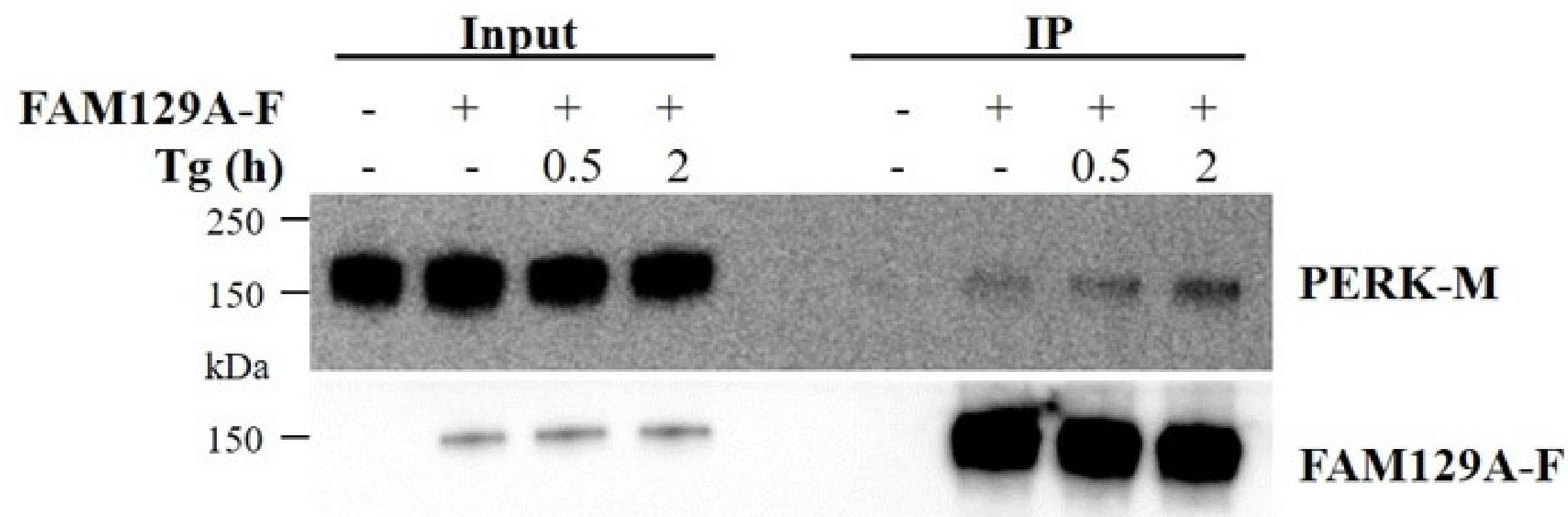

F

G

PERK
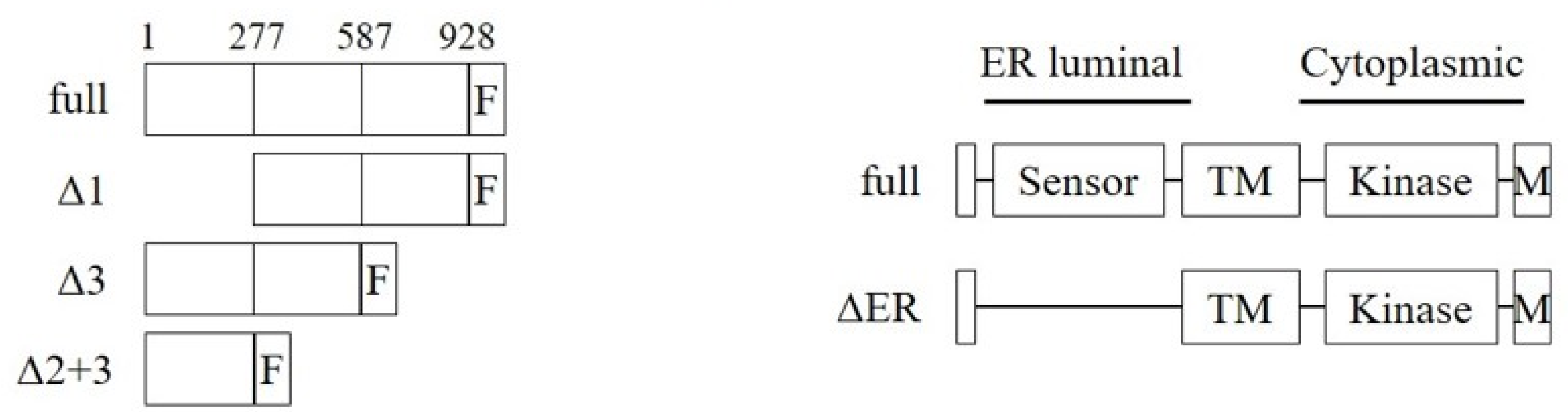

H

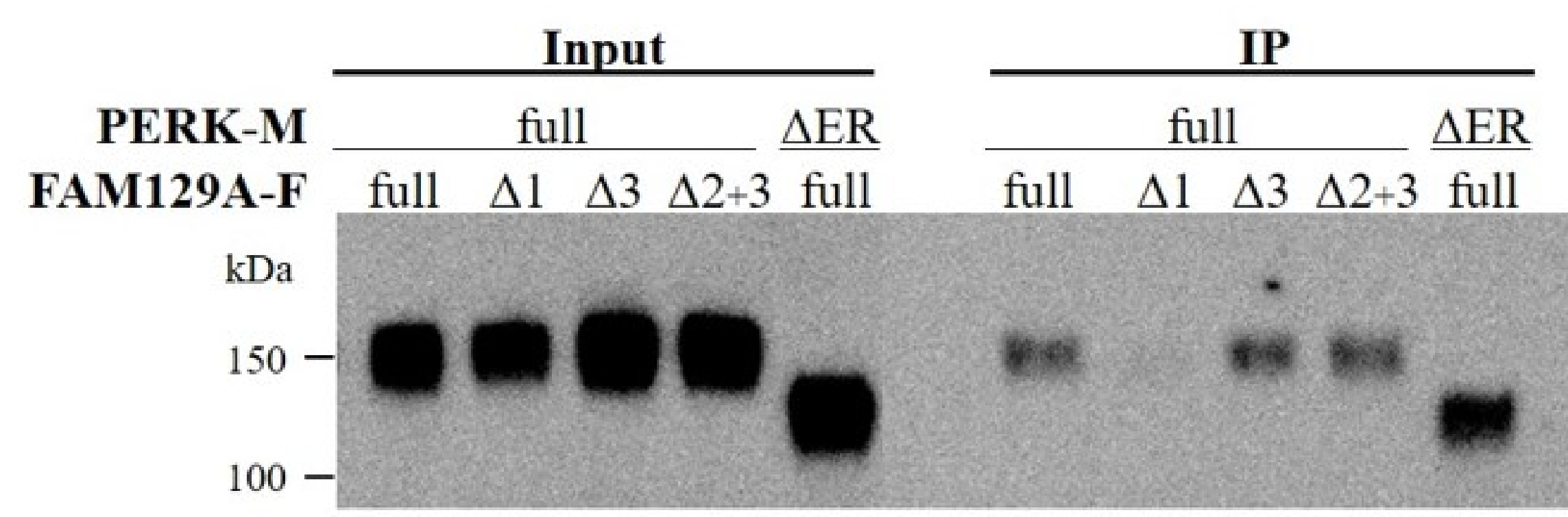

I
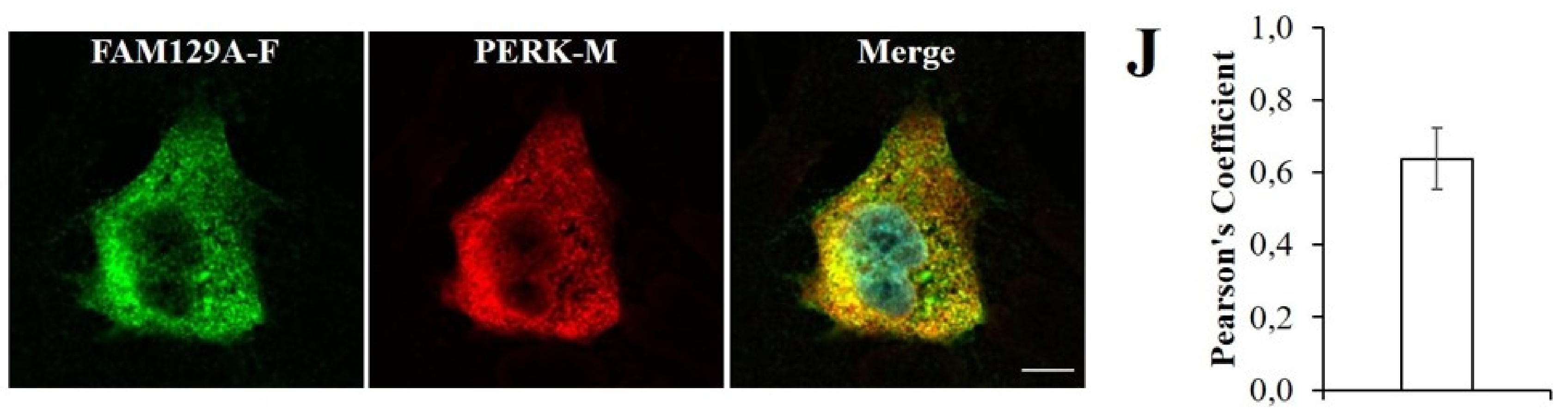


\section{ER stress}

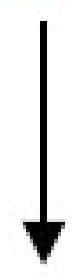

p-PERK

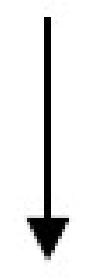

p-eIF $2 \alpha$

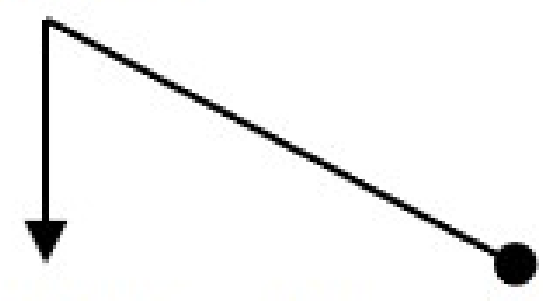

ATF4 Translation

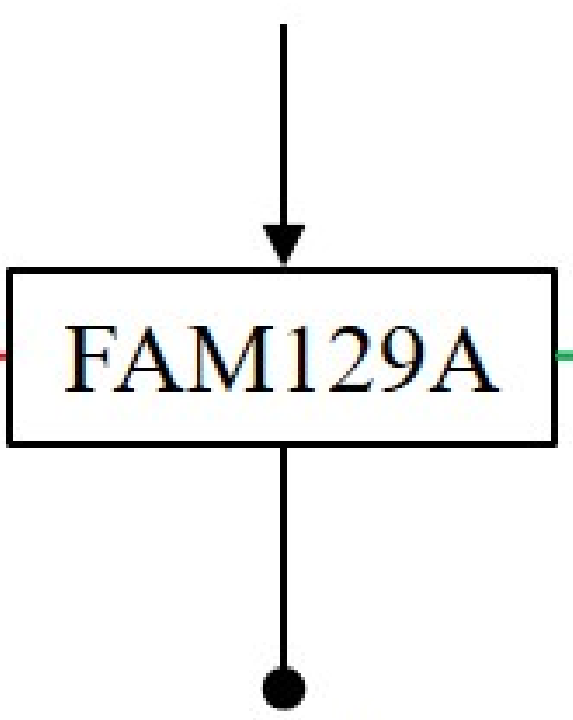

Senescence/Apoptosis 


\section{Supplementary Information}

- Supplemental Figures and their Legends S1-S7

- Supplemental Tables and their Legends S1-S2

- Supplemental Methods 
Supplemental Figures with Legends

A

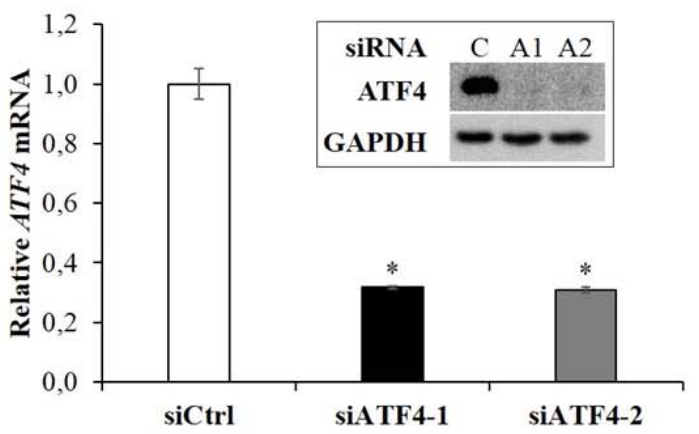

C

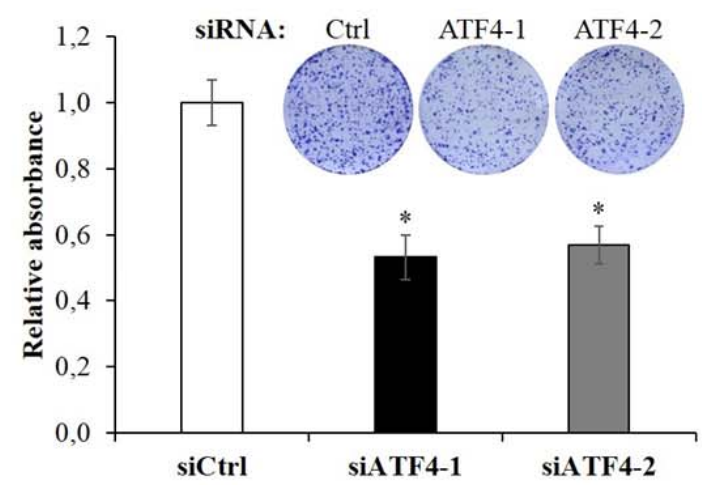

D
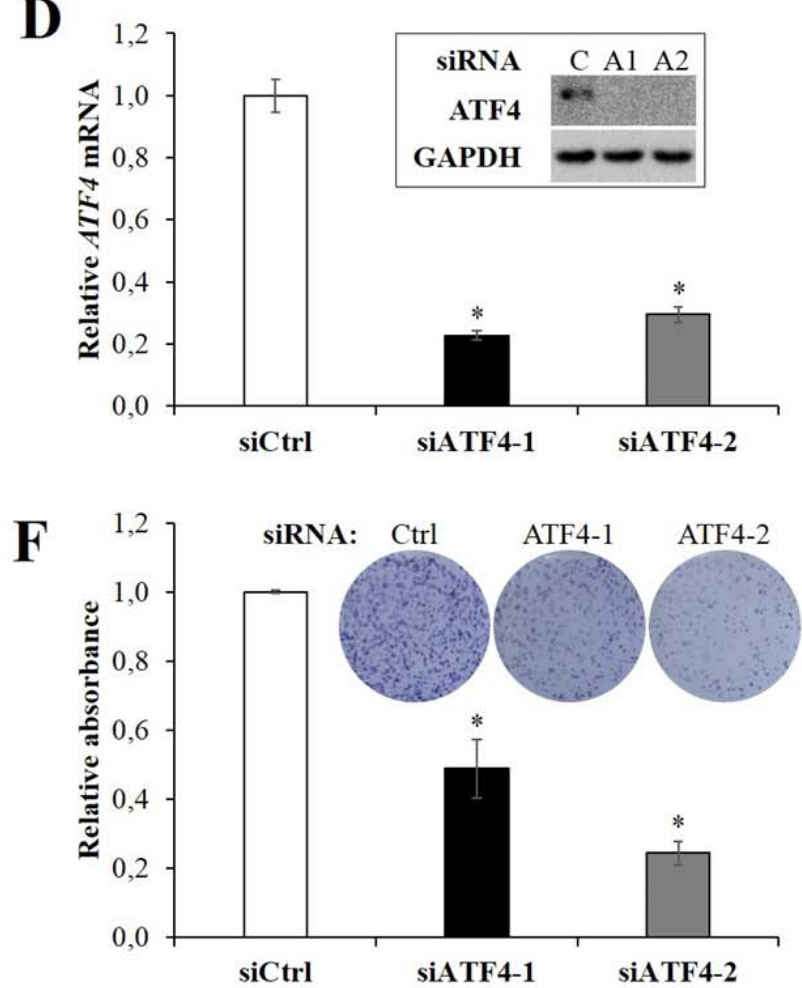

B

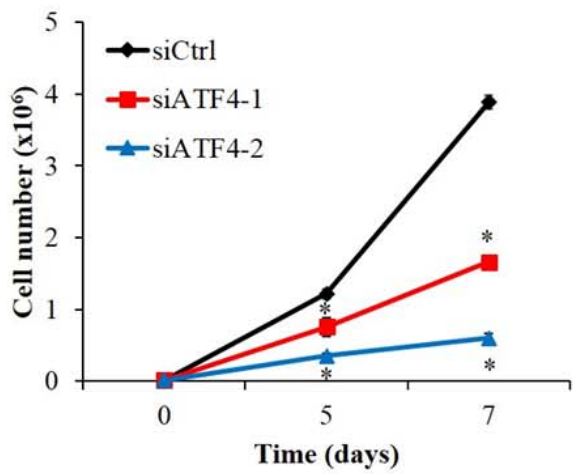

E

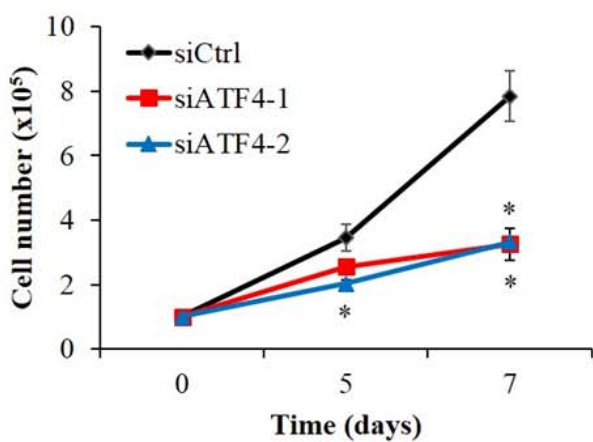


G

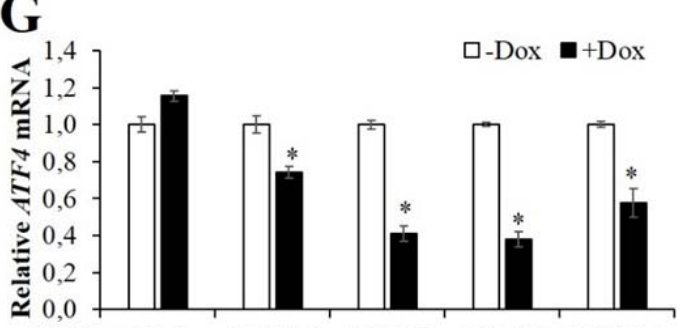

H

shRNA: Ctrl ATF4-1 ATF4-2 ATF4-3 ATF4-4

I

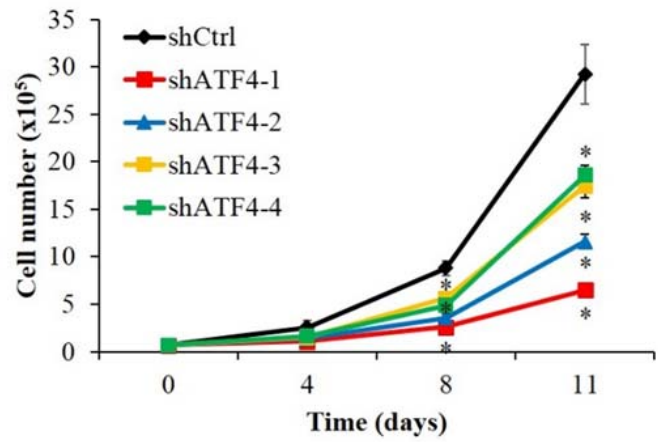

J shRNA: Ctrl ATF4-1 ATF4-2 ATF4-4

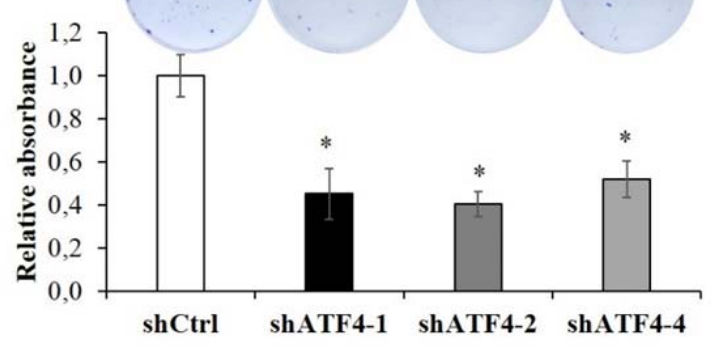

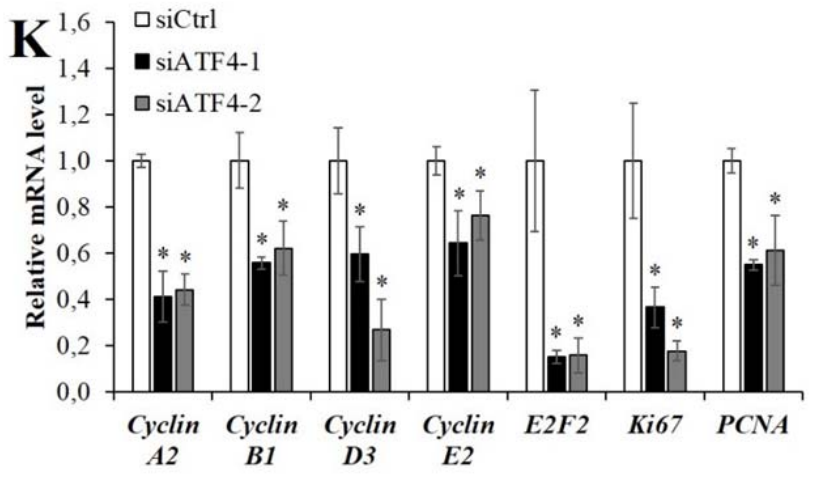


Supplemental Figure S1. ATF4 depletion inhibits growth of PCa cells in vitro.

A) VCaP cells were transfected with either control siRNA or two independent ATF4-specific siRNAs. ATF4 knockdown was confirmed by qPCR and Western analysis upon Tg stimulation. $* p<0,001$.

B) VCaP cells transfected with either control siRNA or two independent ATF4-specific siRNAs were cultured for the indicated times, and cell numbers were determined by trypan blue staining. ${ }^{*} p<0.01$.

C) VCaP cells were transfected with either control siRNA or two independent ATF4-specific siRNAs and cultured for three weeks. Colonies formed were stained and quantified as described in Materials and Methods. ${ }^{*} p<0.05$.

D) 22Rv1 cells were transfected with either control siRNA or two independent ATF4-specific siRNAs. ATF4 knockdown was confirmed by qPCR and Western analysis upon Tg stimulation. $* p<0,0001$.

E) 22Rv1 cells transfected with either control siRNA or two independent ATF4-specific siRNAs were cultured for the indicated times, and cell numbers were determined by trypan blue staining. ${ }^{*} p<0.05$.

F) 22Rv1 cells were transfected with either control siRNA or two independent ATF4-specific siRNAs and cultured for two weeks. Colonies formed were stained and quantified as described in Materials and Methods. ${ }^{*} \mathrm{p}<0.05$.

G) LNCaP cells were transduced with either doxycycline-inducible control shRNA (shCtrl) or four independent doxycycline-inducible ATF4-specific shRNAs (shATF4-1, -2, -3, and -4). Cells were treated with $1.0 \mu \mathrm{g} / \mathrm{mL}$ Dox for $72 \mathrm{~h}$ and ATF4 knockdown was confirmed by qPCR upon $\mathrm{Tg}$ stimulation. ${ }^{*} p<0,01$.

H) ATF4 knockdown in LNCaP cells transduced and treated as described in G was confirmed by Western analysis upon $\mathrm{Tg}$ stimulation.

I) LNCaP cells stably expressing inducible shRNA against ATF4 or control shRNA were treated with Dox, cultured for the indicated times, and cell numbers were determined by trypan blue staining. ${ }^{*} p<0.01$.

J) LNCaP cells stably expressing inducible shRNA against ATF4 or control shRNA were treated with Dox and cultured for three weeks. Colonies formed were stained and quantified as described in Materials and Methods. ${ }^{*} p<0.05$.

K) ATF4 knockdown decreases cell cycle related gene expression. LNCaP cells that were transfected with either control siRNA or two independent ATF4-specific siRNAs were synchronized by starvation for $48 \mathrm{~h}$ and then released into the cell cycle for $24 \mathrm{~h}$. Expression of cell cycle related genes was analyzed by qPCR upon $\operatorname{Tg}$ stimulation. ${ }^{*} p<0,01$. 
A MA data

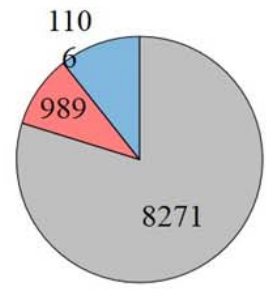

MS data I

3551 proteins

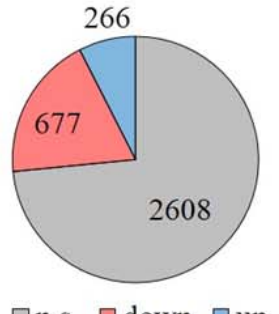

$\square$ n.s. $\square$ down $\square$ up
MS data II

3379 proteins

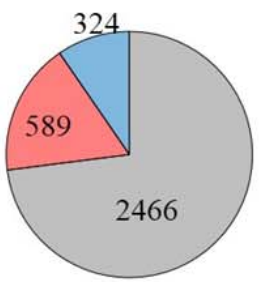

C
B

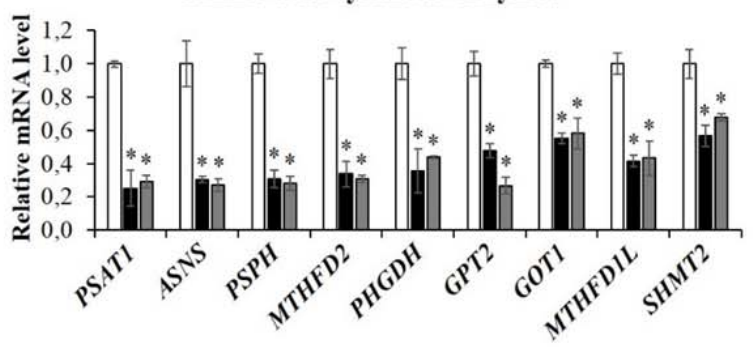

Amino acid transporter

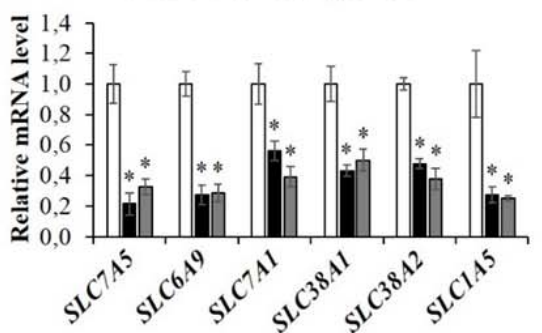

D

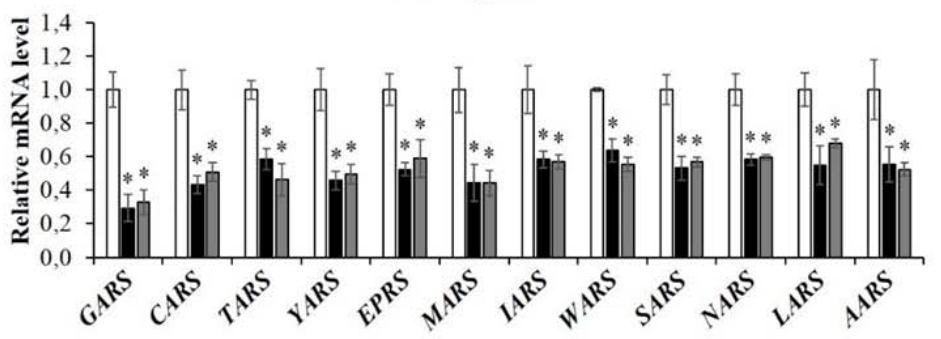

E

F

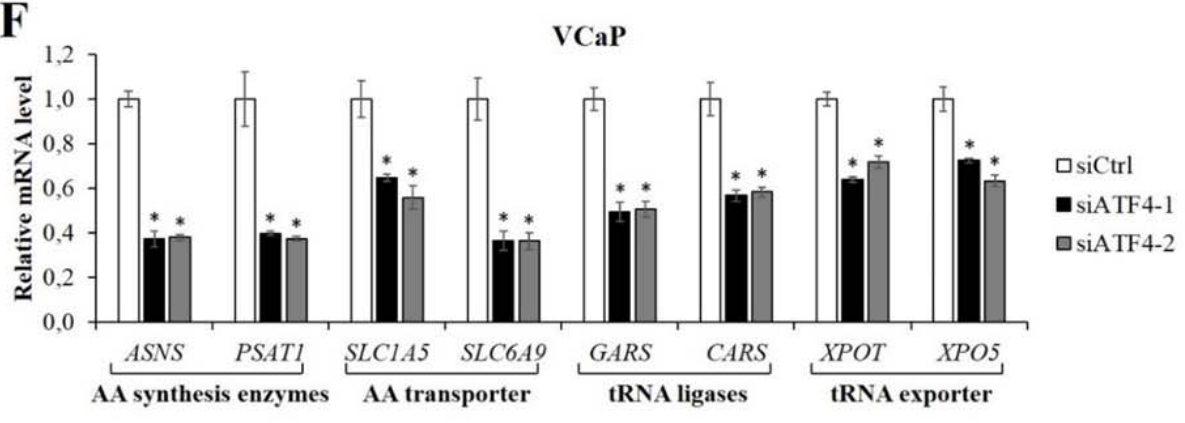

G

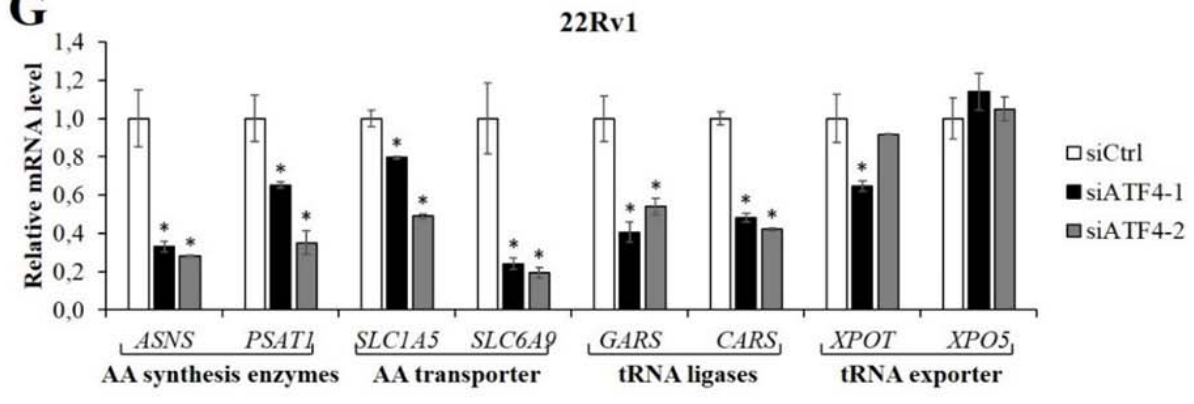




\section{H}

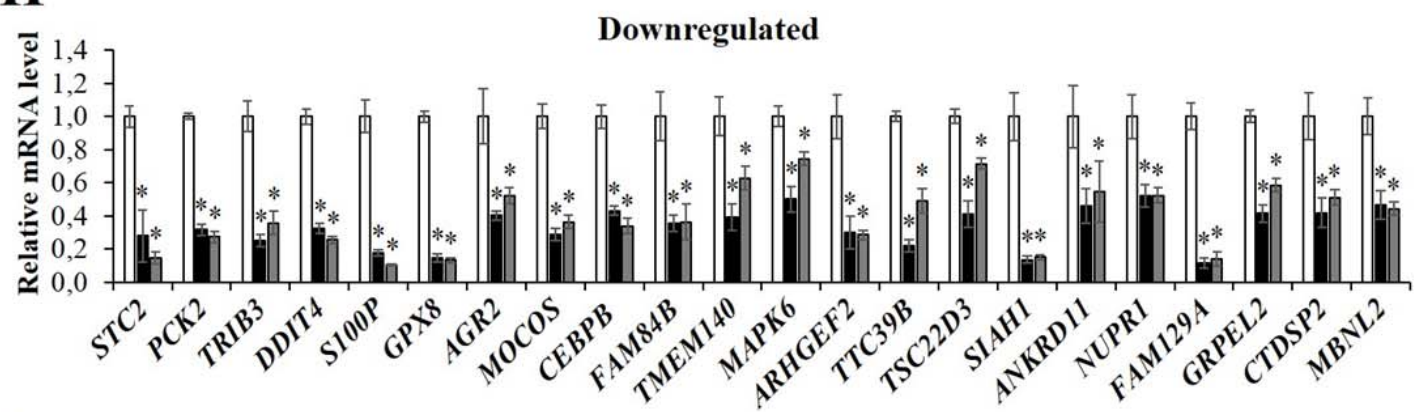

I
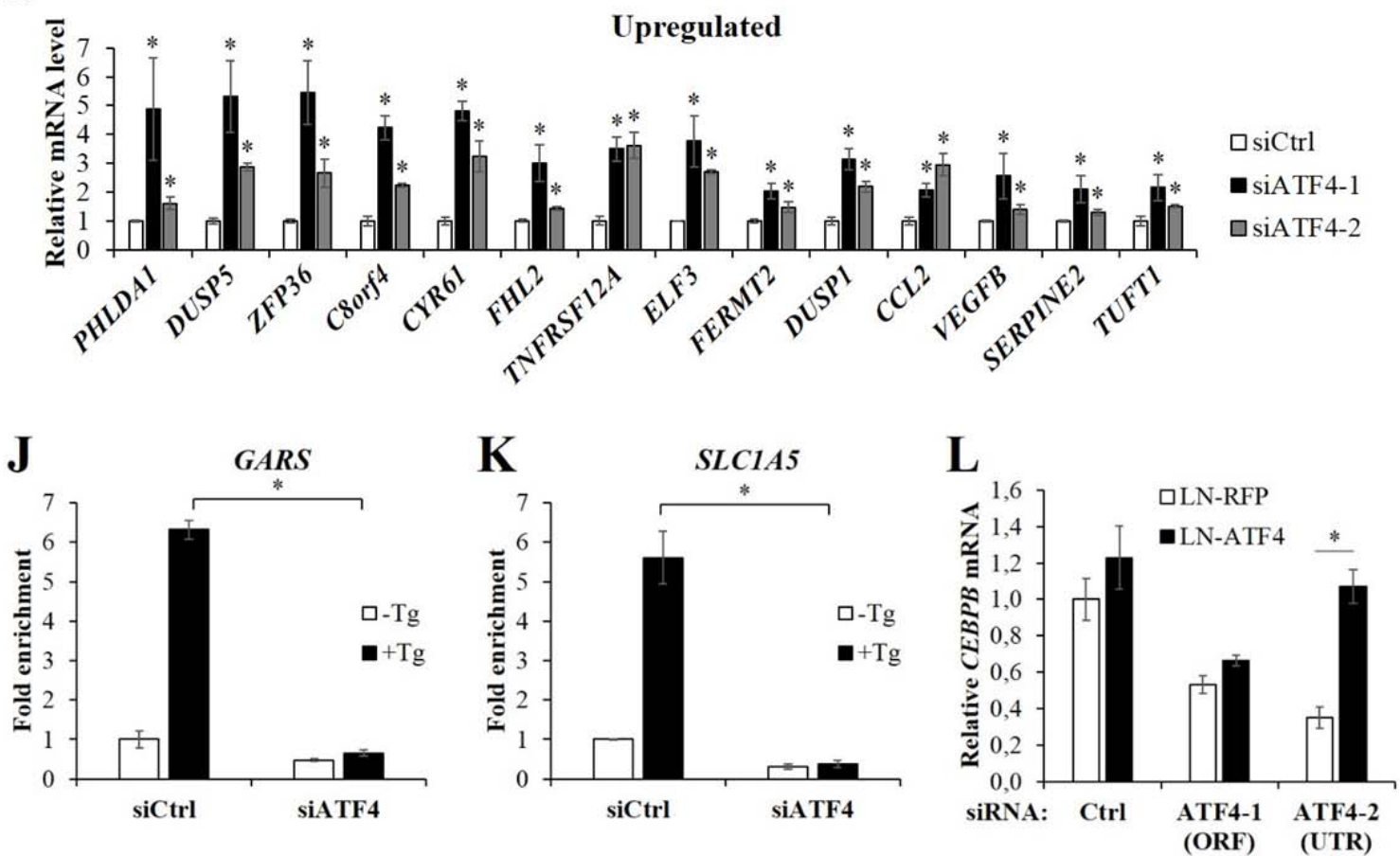

M

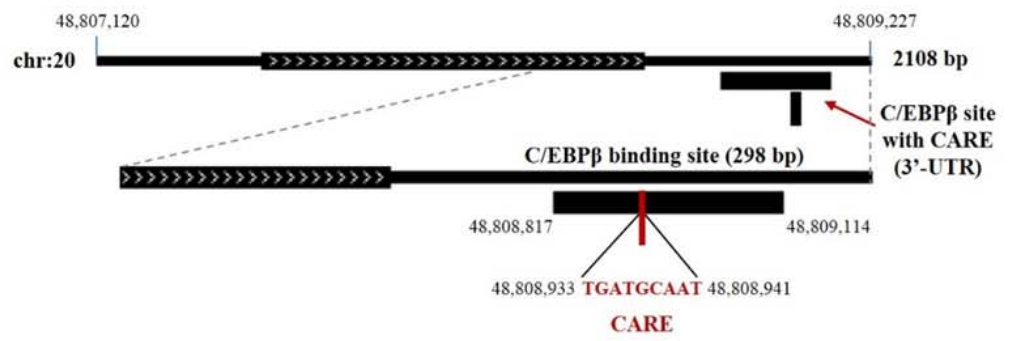


Supplemental Figure S2. Global transcriptomic and proteomic gene expression analysis upon ATF4 depletion in PCa cells.

A) LNCaP cells were transfected with control siRNA or an ATF4-specific siRNA and global transcriptomic (MA) and proteomic (MS) gene expression analysis was performed. The figure indicates the number of downregulated and up-regulated genes, and those where the effects were non-significant (n.s.), upon loss of ATF4 expression; the total number of identified genes/proteins in each array is also shown. MA and MS-I analyses were performed after $5 \mathrm{~h}$ of Tg induction, MS-II analysis after $12 \mathrm{~h}$ of Tg induction. Proteins with fold change $\leq 0.5$ and genes with $\log \mathrm{FC}<0$ and $\mathrm{p}<0,05$ were considered significantly downregulated. Proteins with fold change $\geq 2$ and genes with $\operatorname{logFC}>0$ and $p<0,05$ were considered significantly upregulated.

B-E) ATF4 knockdown decreases expression of amino acid synthesis (B), amino acid transport (C), tRNA ligase (D), and tRNA exporter (E) genes. Expression in LNCaP cells transfected with either control siRNA or two independent ATF4-specific siRNAs were analyzed by qPCR upon Tg stimulation (300 nM). ${ }^{*} p<0,01$.

F-G) ATF4 knockdown decreases expression of amino acid synthesis, amino acid transport, tRNA ligase, and tRNA exporter genes. Gene expression in VCaP (F) and 22Rv1 (G) cells transfected with either control siRNA or two independent ATF4-specific siRNAs was analyzed by qPCR upon Tg stimulation. $* p<0,01$.

$\mathrm{H}-\mathrm{I})$ ATF4 knockdown decreases $(\mathrm{H})$ or increases $(\mathrm{I})$ the expression of a broad range of genes in PCa cells. Expression in LNCaP cells transfected with either control siRNA or two independent ATF4-specific siRNAs was analyzed by qPCR upon Tg stimulation (300 nM). ${ }^{*} p<0,05$.

J-K) ChIP analysis shows ATF4 binding to a CARE sequence in the intronic region of GARS (J) and SLC1A5 (K). LNCaP cells were treated with vehicle or Tg, fixed, and ChIP assay was performed as described in Materials and Methods using an ATF4-specific antibody. The figure shows the average of three independent experiments performed in duplicate. ${ }^{*} p<0,00001$.

L) Downregulation of CEBPB upon ATF4 knockdown is rescued by ATF4 re-expression. LNCaP cells stably expressing the ORF of ATF4 or RFP were transfected with either control siRNA or two independent siRNAs targeting the ORF (siATF4-1) or the 5'-UTR (siATF4-2) of the ATF4 gene and CEBPB expression was analyzed by qPCR upon Tg stimulation. LN-ATF4 cells were protected against ATF4 knockdown mediated downregulation of CEBPB by siATF4-2, but not by siATF4-1. ${ }^{*} p<0,001$.

M) Schematic overview and details of the CARE sequence within a C/EBP $\beta$ binding site in the human CEBPB gene. 

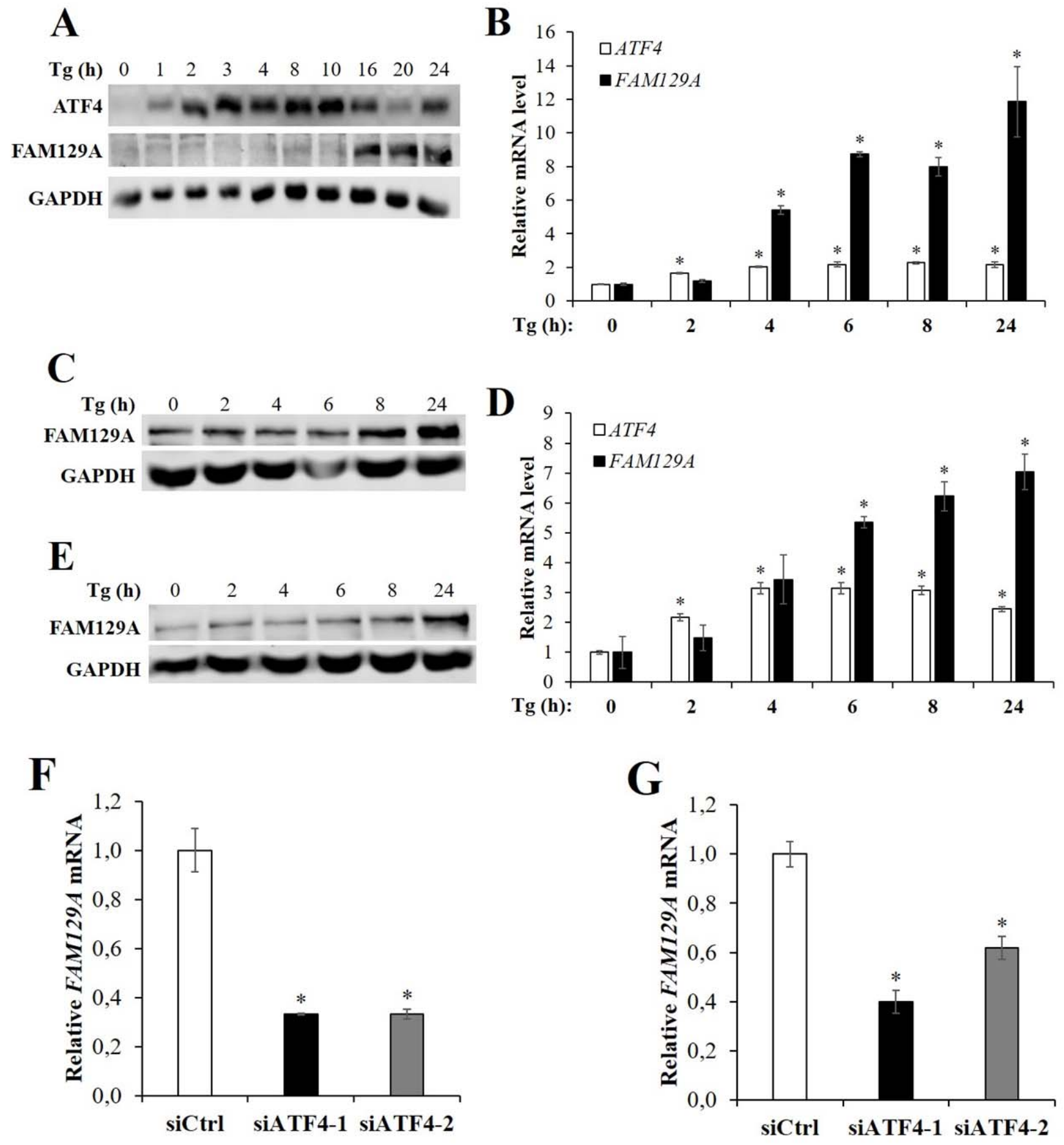
Supplemental Figure S3. FAM129A is induced in PCa cells upon ER stress and regulated by ATF4.

A) Induction of FAM129A expression in LNCaP cells treated with Tg was confirmed by Western analysis.

B) In VCaP cells, FAM129A expression is increased upon Tg-mediated ER stress induction. VCaP cells were treated with Tg for up to $24 \mathrm{~h}$ and expression of FAM129A and ATF4 was analyzed by $q$ PCR. ${ }^{*} p<0,01$.

C) Induction of FAM129A expression in VCaP cells treated with Tg was confirmed by Western analysis.

D) In 22Rv1 cells, FAM129A expression is increased upon Tg-mediated ER stress induction. 22Rv1 cells were treated with Tg for up to $24 \mathrm{~h}$ and expression of FAM129A and ATF4 was analyzed by $q P C R .{ }^{*} p<0,01$.

E) Induction of FAM129A expression in 22Rv1 cells treated with Tg was confirmed by Western analysis.

F) In VCaP cells, ATF4 knockdown decreases FAM129A expression. VCaP cells were transfected with either control siRNA or two independent ATF4-specific siRNAs, treated with $\mathrm{Tg}$, and FAM129A expression was analyzed by qPCR. ${ }^{*} p<0,01$.

G) In 22Rv1 cells, ATF4 knockdown decreases FAM129A expression. 22Rv1 cells were transfected with either control siRNA or two independent ATF4-specific siRNAs, treated with $\mathrm{Tg}$, and FAM129A expression was analyzed by $\mathrm{qPCR} .{ }^{*} \mathrm{p}<0,01$. 
A

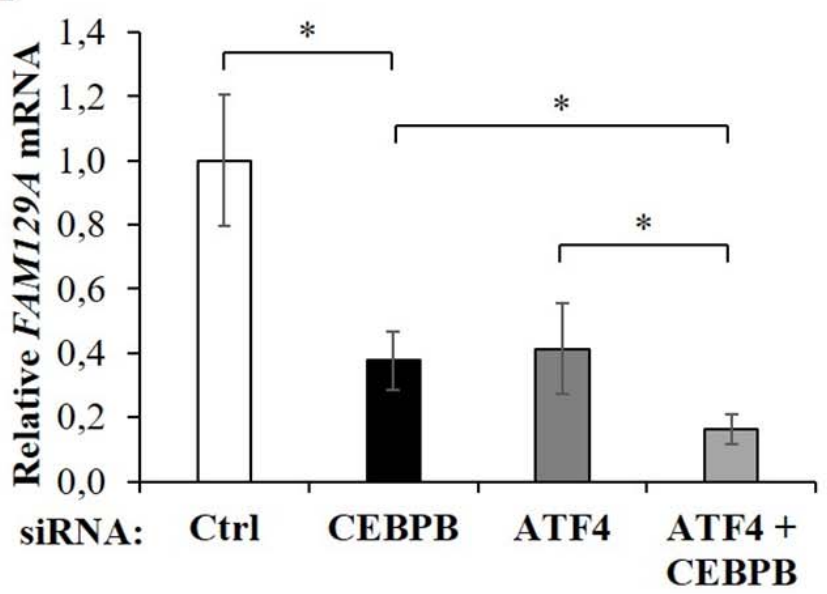

B

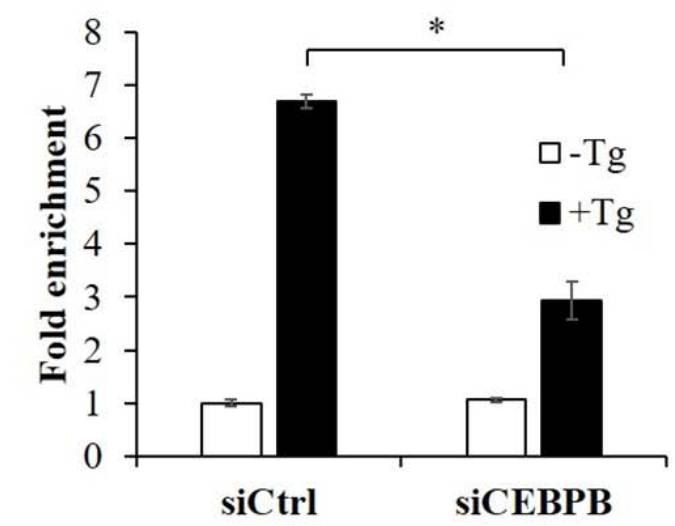

C

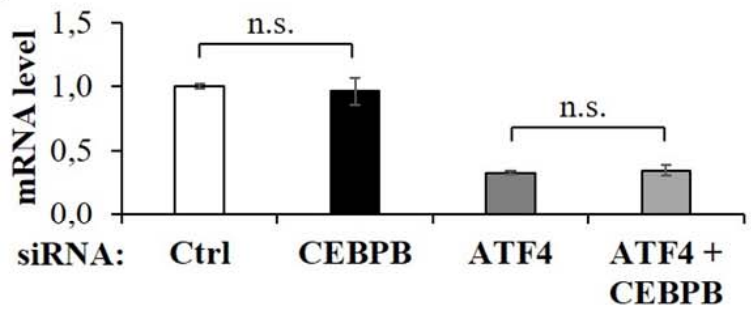

GARS

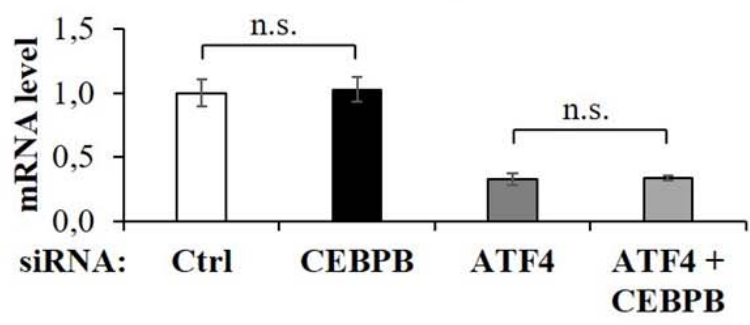

D

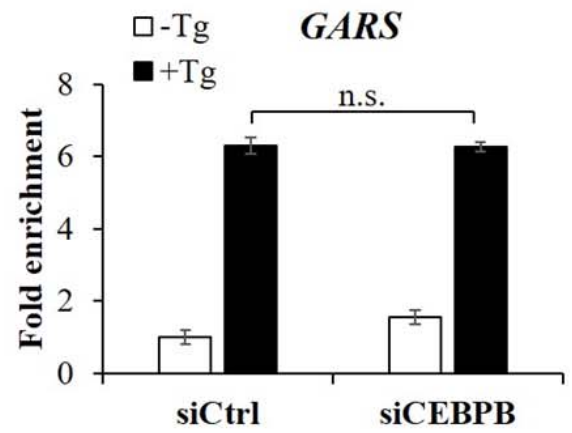

SLC1A5

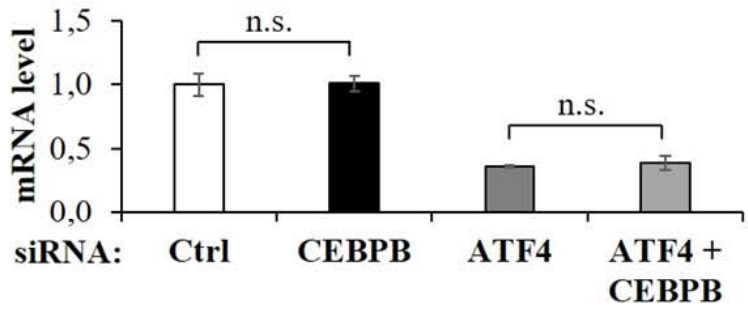

XPO5

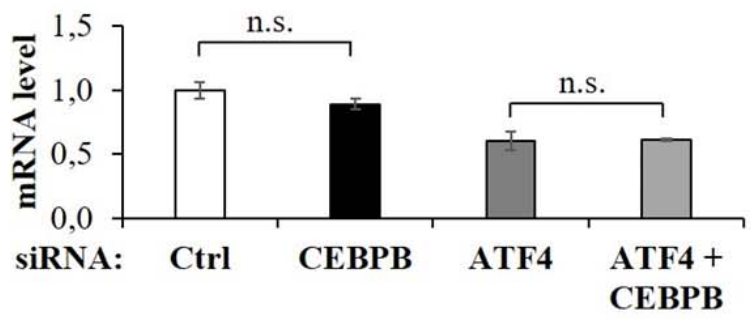

E

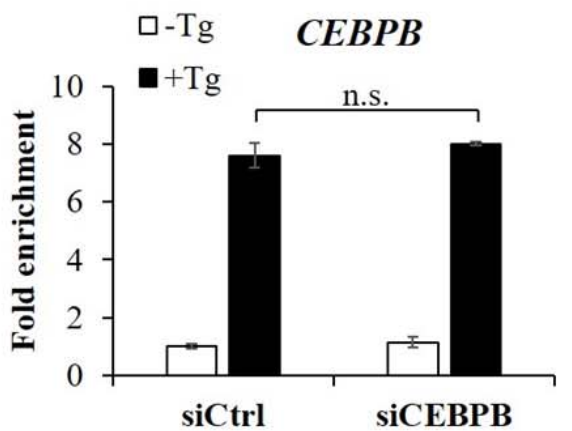


Supplemental Figure S4. C/EBP $\beta$ is required for transcriptional regulation of FAM129A by ATF4.

A) C/EBP $\beta$ knockdown decreases FAM129A expression and has an additive effect to ATF4 knockdown. LNCaP cells were transfected with either control siRNA, CEBPB-specific siRNA (siCEBPB), ATF4-specific siRNA or a combination of both siRNAs as described in Material and Methods, treated with Tg, and FAM129A expression was analyzed by qPCR. * $p<0,05$.

B) C/EBP $\beta$ knockdown reduces ATF4 binding to the CARE sequence in the FAM129A gene. LNCaP cells were transfected with either control siRNA or a CEBPB-specific siRNA, treated with vehicle or Tg, fixed, and ChIP assay was performed as described in Materials and Methods using an ATF4-specific antibody. ${ }^{*} p<0,01$.

C) C/EBP $\beta$ knockdown does not decrease expression of a selection of known ATF4 target genes. LNCaP cells were transfected with a reduced amount of either control siRNA, CEBPB-specific siRNA, ATF4-specific siRNA or a combination of both siRNAs as described in Material and Methods, treated with Tg, and expression of PSAT1, SLC1A5, GARS and XPO5 was analyzed by qPCR.

D-E) C/EBP $\beta$ knockdown does not reduce ATF4 binding to the CARE sequence in the GARS (D) or CEBPB (E) genes. LNCaP cells were transfected with either control siRNA or a CEBPB-specific siRNA, treated with vehicle or $\mathrm{Tg}$, fixed, and ChIP assay was performed as described in Materials and Methods using an ATF4-specific antibody. 
A

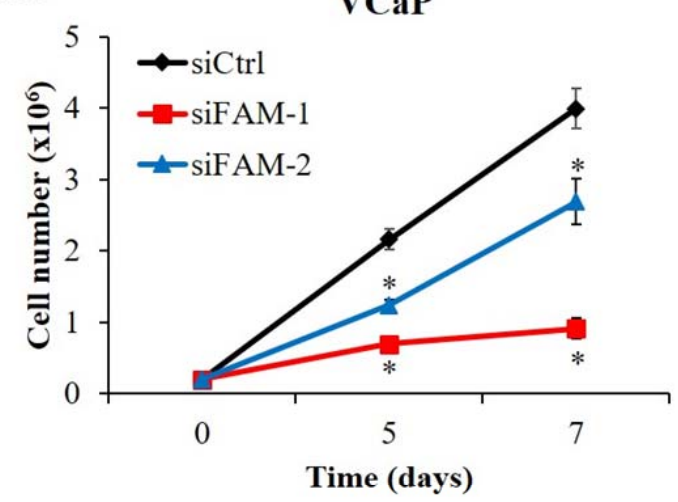

C

siCtrl

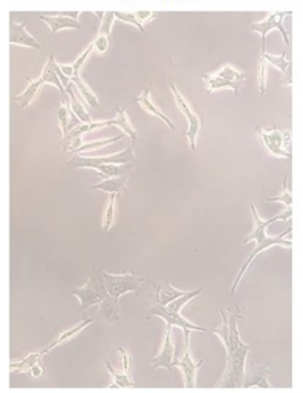

SiFAM-1

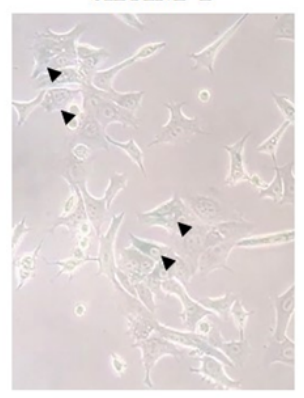

B

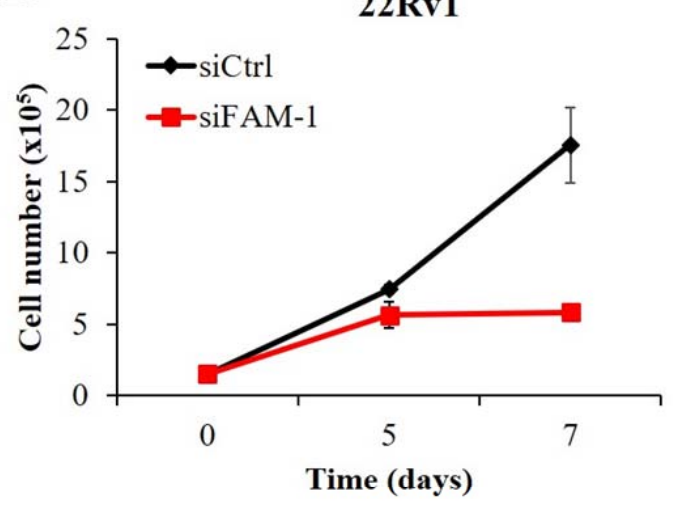

SiFAM-2

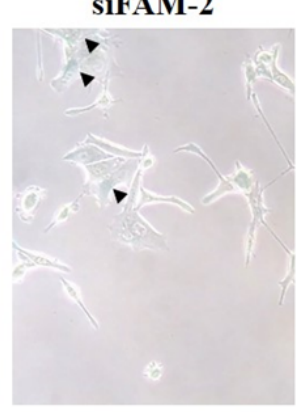

D

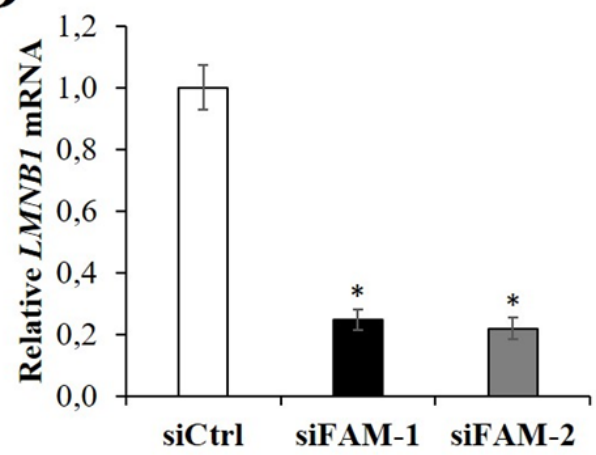

Supplemental Figure S5. FAM129A depletion inhibits PCa cell growth and induces senescence.

A-B) FAM129A knockdown decreases PCa cell growth. VCaP (A) and 22Rv1 (B) cells were transfected with either control siRNA or two independent FAM129A-specific siRNAs and cultured for the indicated times. Cell numbers were then determined by trypan blue staining. $*_{p}<0.01$.

C) FAM129A depletion increases SAßGal activity. LNCaP cells were transfected with either control siRNA or two independent FAM129A-specific siRNAs and were fixed 6 days later, and SAßGal assay was performed. Arrow heads mark cells showing blue staining.

D) LMNB1 expression is decreased upon FAM129A depletion. LNCaP cells transfected with either control siRNA or two independent FAM129A-specific siRNAs were synchronized by starvation for $48 \mathrm{~h}$ and then released into the cell cycle for $24 \mathrm{~h}$. LNMB1 expression was analyzed 6 days after transfection by qPCR upon Tg stimulation. ${ }^{*} p<0,001$. 

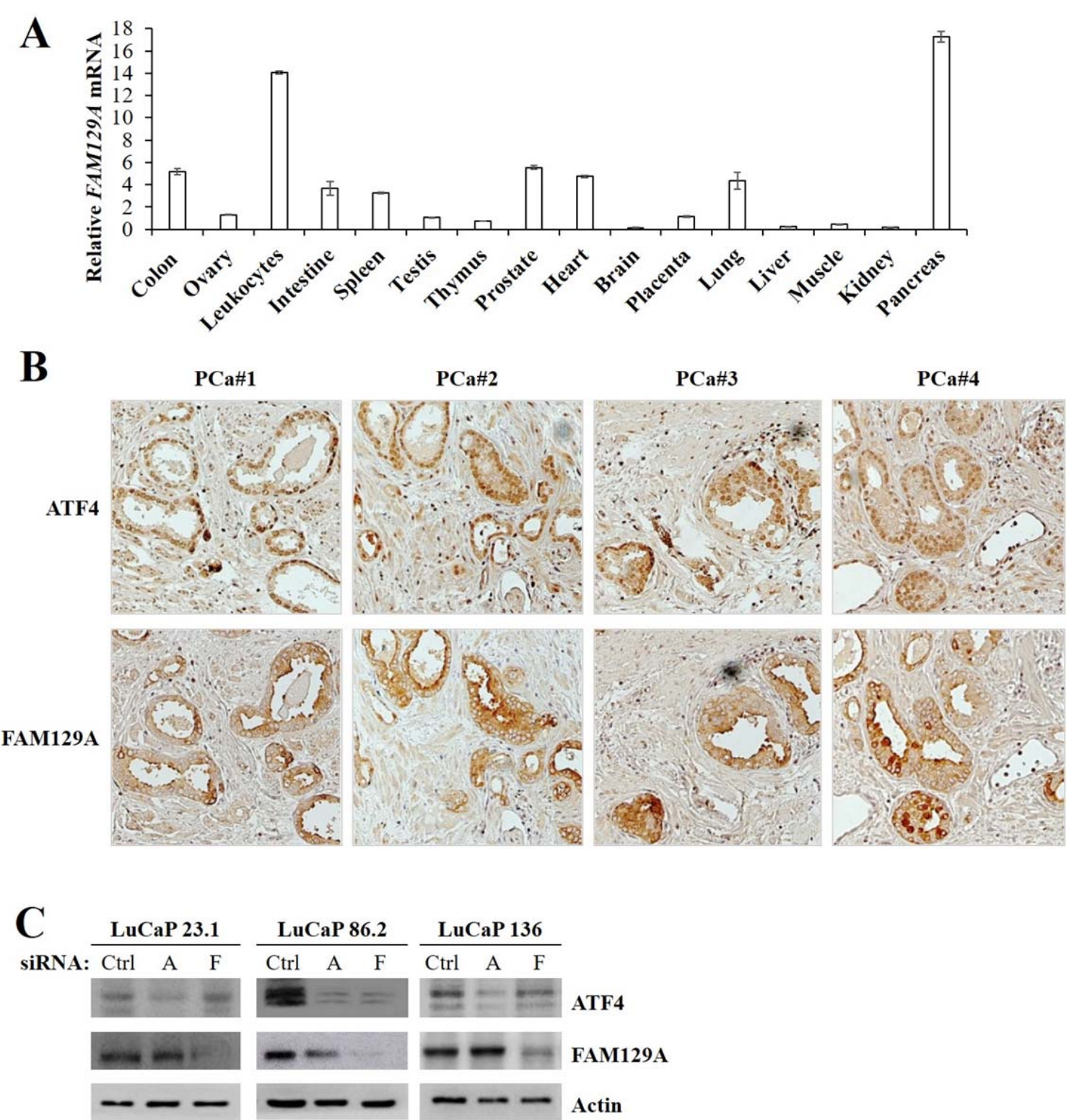


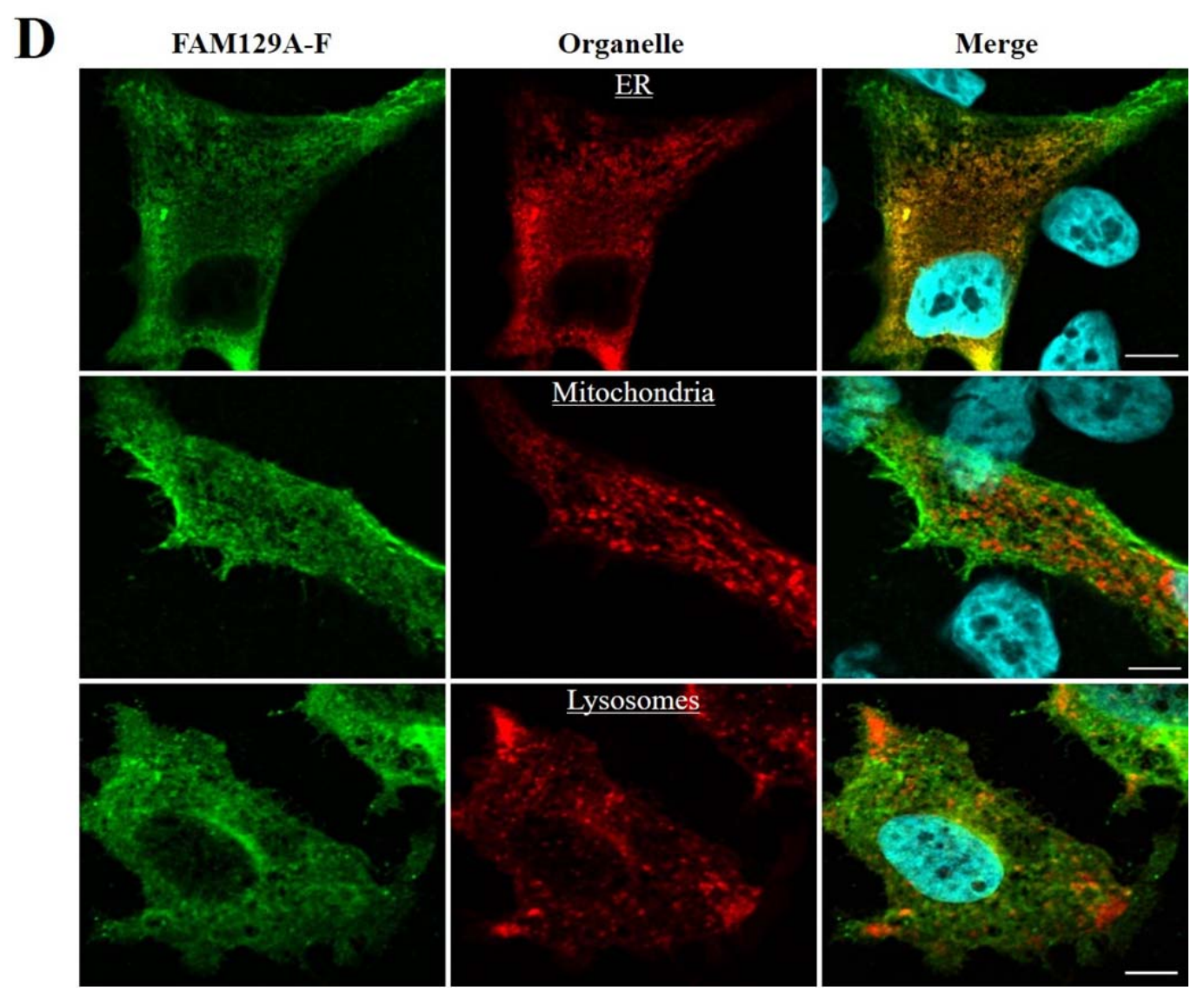

Supplemental Figure S6. FAM129A expression in diverse normal human tissues, prostate cancer specimens, patient-derived organoids and intracellular localization.

A) FAM129A shows a medium-high mRNA expression in human prostate tissue. FAM129A expression in a human cDNA panel was analyzed by qPCR.

B) Colocalization of ATF4 and FAM129A in PCa specimens. Immunohistochemistry was used to assess ATF4 and FAM129A expression on consecutive prostate cancer sections.

C) LuCaP $(23.1,86.2$ and 136) organoids were grown on Nunclon Sphera ultra low attachment 6-well plates and transfected with 100nM of control (Ctrl), ATF4- (A) or FAM129A- (F) specific siRNAs. Protein levels of ATF4 and FAM129A after 3 days of siRNA treatment were determined by western analysis.

D) FAM129A colocalizes with ER marker. LNCaP ectopically expressing FAM129A-FLAG (FAM129A-F) and DsRed-ER-5 (ER), EGFP-LAMP1 (Lyso), or mCherry-mito-7 (Mito) were subjected to confocal immunofluorescence and subsequent colocalization analysis. The scale bar is $5 \mu \mathrm{m}$. 
A
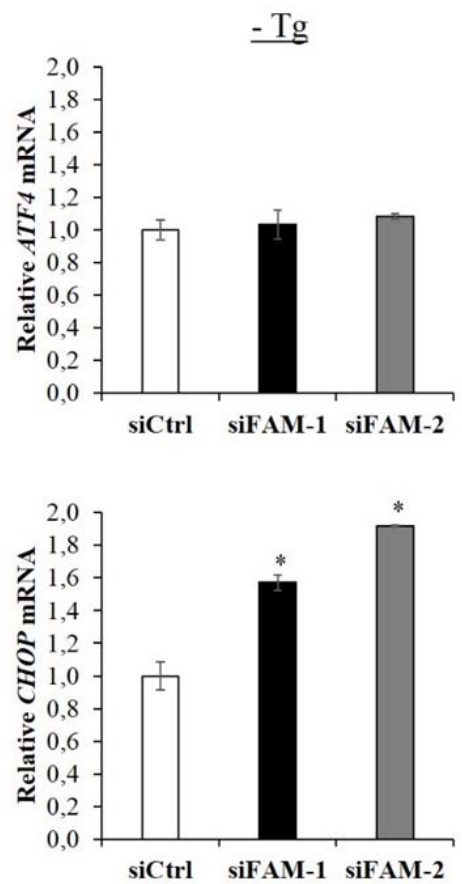
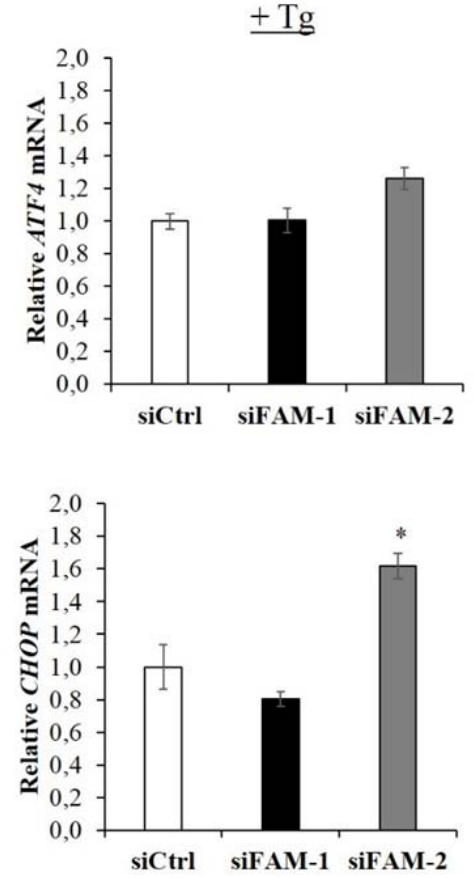

B

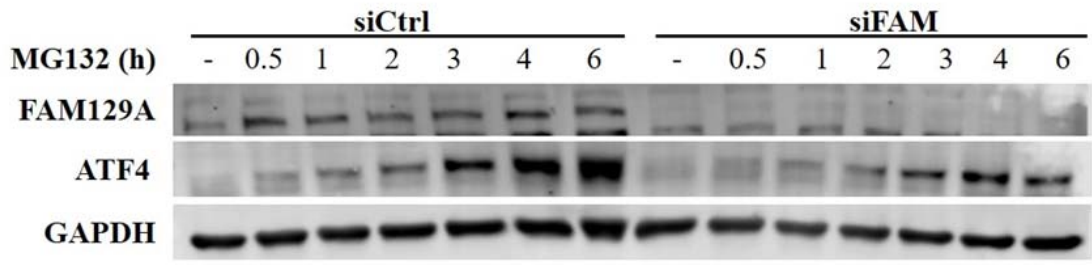

C

\begin{tabular}{r|cccccc} 
Dox $(\mathrm{mg} / \mathrm{mL})$ & 0 & 0.25 & 0.5 & 1.0 & 1.5 & 2.0 \\
FAM129A & & & & & \\
p-PERK & & \\
GAPDH & &
\end{tabular}



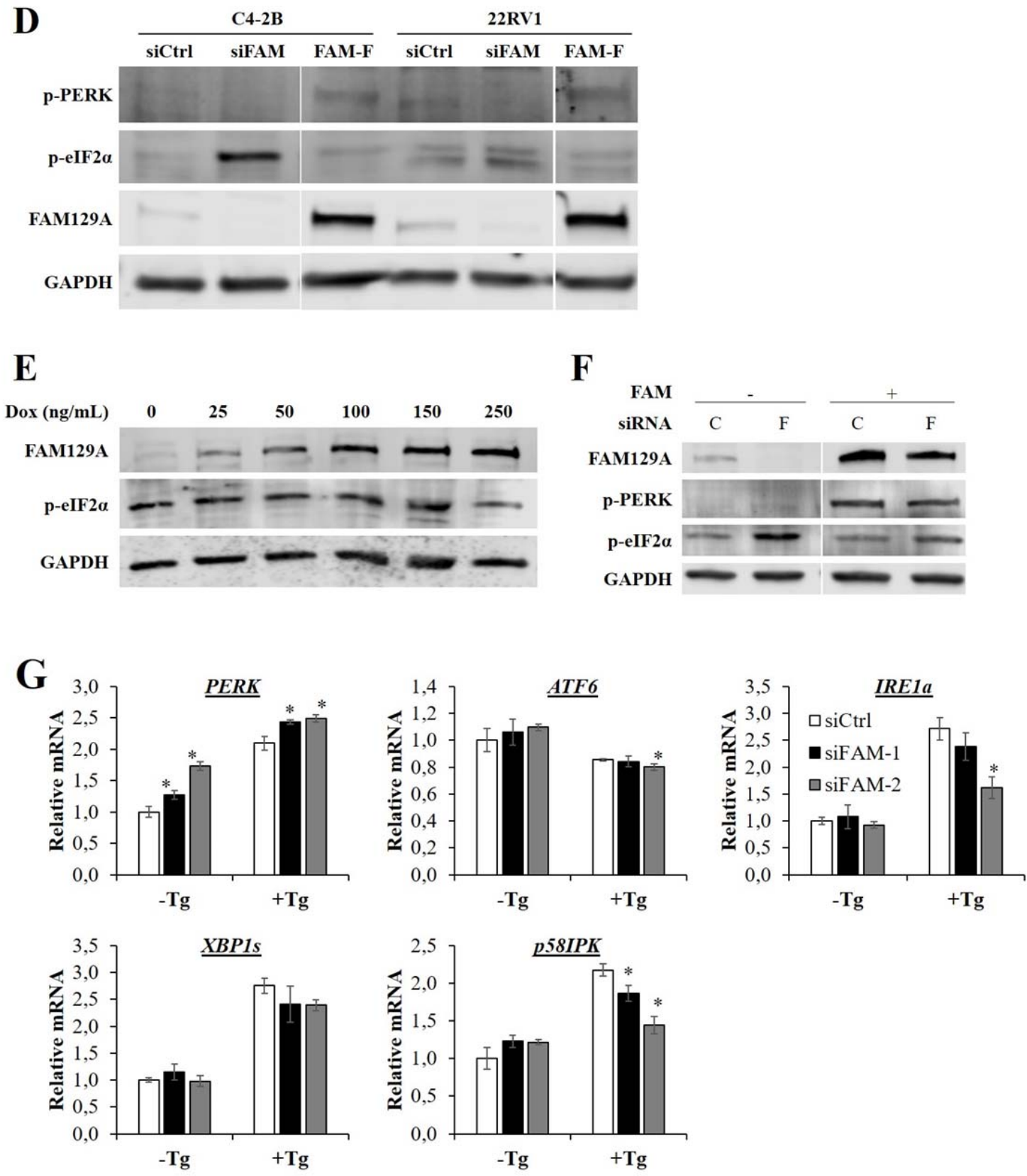

Supplemental Figure S7. FAM129A differentially affects PERK and elF2 $\alpha$ activities.

A) FAM129A knockdown does not decrease ATF4 and CHOP expression. LNCaP cells were transfected with either control siRNA or two independent FAM129A-specific siRNAs and ATF4 and CHOP expression was analyzed 6 days after transfection by qPCR with or without Tg stimulation. ${ }^{*} p<0,01$.

B) LNCaP cells were transfected with either control siRNA or FAM129A-specific siRNA, treated with MG132 for the indicated times. Expression of FAM129A and ATF4 was determined by Western analysis. 
C) FAM129A increases p-PERK levels. LNCaP cells were transduced with doxycycline (Dox)inducible FAM129A expression vector and treated with the indicated amounts of Dox for six days. FAM129A expression and PERK phosphorylation were assessed by Western analysis upon $\mathrm{Tg}$ stimulation.

D) FAM129A silencing increases p-elF2 $\alpha$ level, while FAM129A expression increases p-PERK levels. C4-2B and 22Rv1 cells were transfected with either control siRNA, FAM129A-specific siRNA or a FAM129A expression vector. FAM129A expression and phosphorylation of PERK and elF $2 \alpha$ were determined three days later by Western analysis upon Tg treatment.

E) elF2 $\alpha$ phosphorylation is not appreciably changed upon FAM129A expression. Same as in C. FAM129A expression and elF2 $\alpha$ phosphorylation were assessed by Western analysis upon $\mathrm{Tg}$ stimulation.

F) The FAM129A-mediated increase in p-PERK level is decreased by FAM129A inhibition. LNCaP cells were transfected with an expression vector specifying FAM129A together with either control siRNA or FAM129A-specific siRNA. FAM129A, p-PERK, and p-elF2 $\alpha$ expression was determined six days later by Western analysis upon Tg treatment.

G) FAM129A knockdown differentially affects gene expression of the different UPR branches. LNCaP cells were transfected with either control siRNA or two independent FAM129Aspecific siRNAs and PERK, ATF6, IRE1a, XPB1s and p58IPK mRNA expression was analyzed 3 days after transfection by QPCR with or without Tg stimulation. Results normalized to TBP are presented. ${ }^{*} p<0,01$. 


\section{Supplemental Tables with Legends}

$\underline{\text { Top downregulated genes }}$

\begin{tabular}{cc} 
Symbol & logFC \\
\hline STC2 & $-2,17$ \\
PCK2 & $-1,66$ \\
ATF4 & $-1,61$ \\
TRIB3 & $-1,60$ \\
DDIT4 & $-1,53$ \\
GARS* & $-1,52$ \\
S100P & $-1,46$ \\
PSAT1* & $-1,46$ \\
ASNS* & $-1,42$ \\
SLC7A5 & $-1,39$ \\
GPX8 & $-1,24$ \\
PSPH* & $-1,22$ \\
MTHFD2* & $-1,17$ \\
AGR2 & $-1,17$ \\
MOCOS* & $-1,15$ \\
SLC6A9 & $-1,02$ \\
CEBPB & $-1,02$ \\
FAM84B & $-1,01$ \\
TMEM140 & $-0,99$ \\
SLC7A1* & $-0,99$ \\
SLC38A1 & $-0,98$ \\
MAPK6 & $-0,97$ \\
SLC38A2 & $-0,97$ \\
ARHGEF2* & $-0,95$ \\
TTC39B & $-0,93$ \\
TSC22D3 & $-0,92$ \\
SIAH1 & $-0,90$ \\
ANKRD11 & $-0,88$ \\
NUPR1 & $-0,88$ \\
FAM129A* & $-0,86$ \\
XPOT** & $-0,86$ \\
PHGDH & $-0,85$ \\
GRPEL2** & $-0,84$ \\
CARS* & $-0,83$ \\
CTDSP2 & $-0,81$ \\
MBNL2 & $-0,81$ \\
&
\end{tabular}

\section{$\underline{\text { Top upregulated genes }}$}

\begin{tabular}{cc} 
Symbol & $\operatorname{logFC}$ \\
\hline PHLDA1 & 1,75 \\
DUSP5 & 1,50 \\
ZFP36 & 1,42 \\
C8orf4 & 1,30 \\
CYR61 & 1,28 \\
FHL2 & 1,24 \\
TNFRSF12A & 1,23 \\
ELF3 & 1,18 \\
FERMT2 & 1,16 \\
DUSP1 & 1,14 \\
CCL2 & 0,98 \\
VEGFB & 0,94 \\
SERPINE2 & 0,91 \\
TUFT1 & 0,88
\end{tabular}

Supplemental Table S1. List of genes that are differentially regulated by ATF4 in PCa cells based on global gene expression analysis.

LNCaP cells were transfected with control siRNA or an ATF4-specific siRNA and global transcriptome analysis was performed using microarrays. The table indicates the top downregulated genes $(A)$ with $\log F C<-0.8)$ and top upregulated genes $(B)$ with $\log F C>+0.8$ upon siRNA-mediated ATF4 knockdown in Tg-treated LNCaP cells. Change in expression of all genes upon ATF4 depletion was validated by qPCR using two independent ATF4-specific siRNAs. Only those genes that showed a change in expression with $p<0,05$ are listed. Genes marked with an asterisk were also found to be significantly regulated at the protein level by MS analysis (fold change $<0.5$ or $>2$, respectively). 


\begin{tabular}{cccccccc} 
Gene & Chromosome & Strand & Start & End & Annotation & Distance to TSS (bp) & Sequence (5'-3') \\
\hline ASNS* & 7 & - & $97,501,780$ & $97,501,788$ & 5 '-UTR & 71 & GTTTCATCA \\
PSAT1 & 9 & + & $80,893,398$ & $80,893,406$ & Upstream TSS & 18,657 & ATTGCATCA \\
\hline SLC1A5* & 19 & - & $47,288,159$ & $47,288,168$ & Intron 1 & 3,817 & CTTGCATCA \\
SLC6A9 & 1 & - & $44,497,051$ & $44,497,059$ & 5 '-UTR & 110 & GTTGCATCA \\
SLC6A9 & 1 & - & $44,495,928$ & $44,495,937$ & Intron 1 & 1,233 & GTTACATCA \\
\hline GARS & 7 & + & $30,635,766$ & $30,635,774$ & Intron 1 & 1,589 & TGATGCAAT \\
CARS & 11 & - & $3,078,648$ & $3,078,656$ & 5 '-UTR & 29 & TGATGCAAC \\
\hline XPOT & 12 & + & $64,798,818$ & $64,798,826$ & Intron 1 & 669 & GTTTCATCA \\
XPO5 & 6 & - & $43,538,931$ & $43,538,938$ & Intron 3 & 4,877 & GTTGCATCA
\end{tabular}

Supplemental Table S2. ATF4 activated genes contain CARE sequences near their TSS.

DNA sequences of selected ATF4 activated human genes were screened for C/EBP $\beta$ DNA-binding sites using UCSC hg19 Genome Browser ChIP-seq track. Potential C/EBP $\beta$-binding sites in candidate target genes were further examined for the presence of CARE sequences. Specific binding of ATF4 to CAREs was evaluated by ChIP analysis. Position of CARE DNA sequence in the $5^{\prime}-3^{\prime}$ orientation (+) is shown and the distance from the TSS is stated. Binding sites marked with an asterisk were confirmed by ChIP analysis. 


\section{Supplemental Methods}

Cell culture treatments

For experiments analyzing expression of cell cycle related genes, cells were synchronized at the G0/G1 phase by serum starvation for $48 \mathrm{~h}$ and then released into the cell cycle by re-addition of $10 \%$ FBS and were analyzed after $24 \mathrm{~h}$. For apoptosis experiments, cells were treated with TNF-related apoptosis-inducing factor (TRAIL) (Enzo Life Sciences, \#ALX-522-003-C010) and/or LY294002 (LY) (Invitrogen, \#PHZ1144). Both floating and adherent cells were harvested. For androgen induction experiment, cells were pre-incubated in 5\% charcoal-treated (CT) FBS for $24 \mathrm{~h}$ and treated for indicating time in 5\% CT-FBS containing media with synthetic androgen, R1881 (Sigma-Aldrich, \#R0908). All cell lines were routinely tested and free of mycoplasma contamination.

\section{Patient derived organoids}

LuCaP patient derived xenograft (PDX) lines were maintained in SCID mice under a protocol approved by the Institutional Animal Use and Care Committee (IACUC) of Georgetown University. To develop organoid cultures, PDX (LuCaP 23.1, 86.2 and 136) tissues were harvested from euthanized mice, washed with PBS, and minced with sharp scalpel blade to make small pieces of tumor tissue. Then, $30 \mathrm{ml}$ of advanced DMEM (Gibco), containing 10\%FBS and penicillin-streptomycin were added to the minced tissue and pipetted 50-60 times with Falcon $10 \mathrm{ml}$ pipette to make smaller fragments. Then, it was passed through a 100 micron cell strainer (BD Falcon) and centrifuged at 100Xg for 5 minutes, and repeated twice. The resultant cluster of cells (10-25 cell clusters) were grown in 1:1 Keratinocyte SFM and advanced DMEM containing 5\% FBS, $400 \mathrm{M}$ L-Glutamine, 5ng/ml EGF, $50 \mathrm{~g} / \mathrm{ml}$ BPE, 10nM DHT and penicillin-streptomycin on Nunclon Sphera ultra low attachment 6-well plate. Culture media was replaced every 2 days and after 7 days when the cell clusters were formed round organoids, it was transfected with 100nM of control, ATF4 or FAM129A siRNAs for 3 days using PepMute siRNA transfection reagents (Signagen Laboratories). After 3 days of siRNA transfection, organoids were taken out for Western blot analysis. Separate 6 well plates with organoids were continued to grow in fresh growth media until day 7. Then, LIVE/DEAD ${ }^{\circledR}$ Viability/Cytotoxicity reagents (Thermo Scientific) were added to the media for $30 \mathrm{~min}$ and organoids were photographed under Olympus fluorescent microscope. Representative images from each of the tree replicates were then quantitated by Nikon NIS-Elements software.

\section{RNA interference}

$1.0 \times 10^{5}$ LNCaP cells were seeded per well in a 6-well plate and transfected with 5 nM siRNA using Lipofectamine RNAiMAX (Invitrogen, \#10601435) and RPMI media without supplements. $4.0 \times 10^{5}$ trypsinized VCaP or 22RV1 cells were resuspended in a mix of $10 \mathrm{nM}$ siRNA and Lipofectamine RNAiMAX in RPMI media without supplements and seeded to a $6-\mathrm{cm}$ plate well. For double knockdowns, single siRNA controls were adjusted to the same amount of siRNA by addition of control 
siRNA. For double knockdown of ATF4 and CEBPB, $2.5 \mathrm{nM}$ of each siRNA was used per well. For double knockdown with FAM129A, $2.5 \mathrm{nM}$ of FAM129A-specific siRNA was used per well in combination with the regular amount of the other siRNA. Medium was replaced the next day and cells were analyzed 3 days after transfection unless indicated otherwise. Sequences and source of siRNAs are provided below.

\begin{tabular}{llll} 
siRNA & Company & $\#$ & Target sequence \\
\hline siCtrl & Qiagen & Sl03650318 & Allstar Negative Control siRNA \\
siATF4-1 & Dharmacon & L-005125 & CAGATTGGATGTTGGAGAA, \\
& & CGACTTGGATGCCCTGTTG, \\
& & GAAGAACGAGGCTCTAAAA, \\
siATF4-2 & Qiagen & SI03019345 & CAGATAGGAAGCCAGACTA \\
siCEBPB & Qiagen & SI02777292 & CGGGCCCTGAGTAATCGCTTA \\
& & SI03058062 & CACCCTGCGGAACTTGTTCAA \\
& & SI03022341 & TTCCGTTTCAAGCATTAAGAA \\
& & SI00073640 & CCCGTGGTGTTATTTAAAGAA \\
siFAM-1 & Qiagen & SI04167219 & CACGTGCGCACTGAAGTAGAA \\
siFAM-2 & Qiagen & - & AGAGATTTAACGTCACAGTTT \\
siPKR & Santa Cruz & sc-36263 & N/A \\
siPERK & Santa Cruz & sc-36213 & N/A \\
siGCN2 & Santa Cruz & sc-45644 & N/A
\end{tabular}

Quantitative PCR

mRNA expression in human tissues was assessed by using human cDNA panels (Clontech, \#636742, \#636743). PCR primer sequences are available upon request. A standard curve made from serial dilutions of cDNA was used to calculate the relative amount of the different cDNAs in each sample. The values were normalized to the relative amount of the internal standard GAPDH and TBP or ACTB. Results normalized to GAPDH are presented unless indicated otherwise.

\section{Western blot analysis}

The following antisera were used: phospho-4E-BP1 (\#2855), ATF4 (\#11815), cleaved Caspase-3 (\#9661), Cyclin B1 (\#12231), Cyclin E2 (\#4132), elF2 $\alpha$ (\#9722), GCN2 (\#3302), phospho-elF2 $\alpha$ (\#9721), cleaved PARP (\#9541), phospho-Rb (\#9308), CHOP (\#2895), PKR (\#12297), phospho-PERK (\#3179), PERK (\#3192) (Cell Signaling Technology); GAPDH (\#sc-47274), ß-Actin (\#sc-47778) (Santa Cruz); 
FAM129A (\#21401) (Signalway Antibodies). All antisera were used at a dilution of 1:1,000, except for GAPDH (1:5,000), $\beta$-Actin (1:2,000) and FAM129A (1:500).

\section{Chromatin immunoprecipitation (ChIP)}

LNCaP cells were plated in 15-cm tissue culture plates and cultured as described above. Cells were treated with $30 \mathrm{nM} \mathrm{Tg}$ or vehicle for $5 \mathrm{~h}$ followed by a crosslinking step ( $1 \%$ formaldehyde at $37^{\circ} \mathrm{C}$ ), and a quenching step with $125 \mathrm{mM}$ glycine. Chromatin was sonicated using the Bioruptor sonicator (Diagenode) and was immunoprecipitated with antibodies against ATF4 (Santa Cruz, \#sc-200X) or IgG (Vector Laboratories, \#I-1000). After reversal of crosslinking, immunoprecipitated DNA, as well as input DNA, was quantified by qPCR. PCR primers used are available upon request. Standard curves were created by 10 -fold serial dilutions of an input template.

Immunofluorescence

LNCaP cells were transfected for 48h with FAM129A-FLAG (GeneScript OHu10615C) and DsRed2-ER-5 (addgene 55836), pEGFP-LAMP1 (generous gift from Odmund Bakke, UiO), mCherry-mito-7 (addgene 55102), or PERK-MYC (GeneScript OHu22427C) plasmids using Lipofectamine 3000 reagent. Cells were treated with $\mathrm{Tg}$ for $24 \mathrm{~h}$ and fixed with $4 \%$ Para formaldehyde $(20 \mathrm{~min}$ ), permeabilized with $0.2 \%$ Triton 100X (20 min), blocked in $1 \%$ BSA $(1 \mathrm{~h})$, and probed with primary antibodies $\left(12 \mathrm{~h}, 4^{\circ} \mathrm{C}\right)$ and secondary antibodies conjugated to Alexa Fluor $\left(1 \mathrm{~h}, 20^{\circ} \mathrm{C}\right.$ ). Primary antibodies: anti-FLAG (cst 14793, 1:800) anti-MYC (cst 2276, 1:800). Secondary antibodies (1:500): anti-mouse AF546 (ThermoFischer A11003), anti-rabbit AF488 (ThermoFischer A11008), anti-rabbit AF555 (ThermoFischer A21428). Imaging was done with an Olympus FluoView 1000 upright confocal microscope (Objective 60X, 1.4 NA). Quantification was performed using Fiji (ImageJ) with an object based Pearson's correlation method similar to what has been previously described [1].

\section{References}

1 Moser B, Hochreiter B, Herbst R, Schmid JA. Fluorescence colocalization microscopy analysis can be improved by combining object-recognition with pixel-intensity-correlation. Biotechnol J 2017; 12. 